\title{
Macdonald polynomials in superspace as eigenfunctions of commuting operators*
}

\author{
Olivier Blondeau-Fournier ${ }^{\dagger}$, Patrick Desrosiers ${ }^{\S}$, \\ LuC LAPOINTE ${ }^{ף}$ AND PIERRE MATHIEU ${ }^{\ddagger}$
}

\begin{abstract}
A generalization of the Macdonald polynomials depending upon both commuting and anticommuting variables has been introduced recently. The construction relies on certain orthogonality and triangularity relations. Although many superpolynomials were constructed as solutions of a highly over-determined system, the existence issue was left open. This is resolved here: we demonstrate that the underlying construction has a (unique) solution. The proof uses, as a starting point, the definition of the Macdonald superpolynomials in terms of the Macdonald non-symmetric polynomials via a non-standard (anti)symmetrization and a suitable dressing by anticommuting monomials. This relationship naturally suggests the form of two families of commuting operators that have the defined superpolynomials as their common eigenfunctions. These eigenfunctions are then shown to be triangular and orthogonal. Up to a normalization, these two conditions uniquely characterize these superpolynomials. Moreover, the Macdonald superpolynomials are found to be orthogonal with respect to a second (constant-termtype) scalar product, and its norm is evaluated. The latter is shown to match (up to a $q$-power) the conjectured norm with respect to the original scalar product. Finally, we recall the super-version of the Macdonald positivity conjecture and present two new conjectures which both provide a remarkable relationship between the new $(q, t)$-Kostka coefficients and the usual ones.
\end{abstract}

${ }^{*}$ This article is dedicated to Professor Adriano Garsia on the occasion of his 84th birthday. The authors are extremely grateful to Alain Lascoux for spending time and effort to provide them a detailed outline of the proof of Proposition 16.

†Supported by FRQNT.

${ }^{\ddagger}$ Supported by NSERC.

${ }^{\S}$ Supported by FONDECYT \#1090034 and by CONICYT's anillo ACT56.

`Supported by FONDECYT \#1090016 and by CONICYT's anillo ACT56. 


\section{Introduction}

\subsection{Macdonald superpolynomials and related positivity conjectures}

A candidate for the superspace extension of the Macdonald polynomials Macdonald superpolynomials for short - has been obtained in [7]. Such an extension involves the anticommuting variables $\theta_{1}, \theta_{2}, \ldots$ (with $\theta_{i}^{2}=0$ ), as well as the usual commuting variables $x_{1}, x_{2}, \ldots$ The superspace approach turns out to be a very restrictive framework: each variable $x_{i}$ is considered to be paired with an anticommuting variable $\theta_{i}$, so that symmetric superpolynomials are required to be invariant under the interchange of pairs $\left(x_{i}, \theta_{i}\right) \leftrightarrow\left(x_{j}, \theta_{j}\right)[9]$.

The construction in [7] is presented as a conjecture (a point developed shortly). But the first exploration of the resulting superpolynomials revealed a very rich structure. As expected, this two-parameter $(q, t)$ family of superpolynomials contains, in the appropriate limit $\left(q=t^{\alpha}, t \rightarrow 1\right)$, the Jack superpolynomials [10]. But, what was totally unexpected a priori, is that it contains two versions of the Hall-Littlewood superpolynomials (for $q=0$ and $q \rightarrow \infty)$. Moreover, from each version of the latter, we can define a natural extension of the Schur polynomials (for $t=0$ and $t \rightarrow \infty$ respectively). Both types of Schurs have a positive integral decomposition into the monomial superpolynomials (a property that is not verified for the Jack superpolynomials at $\alpha=1$ ). But what is more remarkable is that, using the $q=t=0$ Schurs, we could conjecture a generalization of the Macdonald positivity conjecture (reviewed below).

The present article, although completely independent, is a continuation of our previous work [7]. It addresses the following issues: the existence of the Macdonald superpolynomials, their relation with the non-symmetric Macdonald polynomials, and their characterization as an eigenvalue problem. As an aside, new conjectures for the Kostka coefficients are presented.

\subsection{The main result: An existence proof}

The conjectural construction of [7] is proved here to be a valid characterization of the Macdonald polynomials in superspace. To clarify this point, we first recall the definition of the ordinary Macdonald polynomials [24].

The Macdonald polynomials $P_{\lambda}=P_{\lambda}(x ; q, t)$, in the variables $x=$ $x_{1}, x_{2}, \ldots$, are characterized by the two conditions:

1) $P_{\lambda}=m_{\lambda}+$ lower terms,

2) $\left\langle\left\langle P_{\lambda} \mid P_{\mu}\right\rangle\right\rangle_{q, t}=0$ if $\lambda \neq \mu$ 
The triangular decomposition refers to the usual dominance order on partitions (see (11) below) and the $m_{\lambda}$ 's are the monomial symmetric functions. The orthogonality relation is defined in the power-sum basis $p_{\lambda}=p_{\lambda_{1}} \cdots p_{\lambda_{\ell}}$, with $p_{r}=\sum_{i \geq 1} x_{i}^{r}$, as

$$
\left\langle\left\langle p_{\lambda} \mid p_{\mu}\right\rangle\right\rangle_{q, t}=z_{\lambda} \delta_{\lambda \mu} \prod_{i=1}^{\ell(\lambda)} \frac{1-q^{\lambda_{i}}}{1-t^{\lambda_{i}}}, \quad z_{\lambda}=\prod_{i \geq 1} i^{n_{\lambda}(i)} n_{\lambda}(i) !
$$

$n_{\lambda}(i)$ being the number of parts in $\lambda$ equal to $i$. Since the dominance ordering is partial (for degrees $\geq 6$ ), the orthogonality constraint leads to an overdetermined system at each degree $\geq 6$. It is therefore necessary to show that there exists such a family of polynomials. This is generally done through an eigenvalue-problem characterization.

The brute-force approach followed in [7] was to look for a suitable deformation of the scalar product (2) that allows for nontrivial solutions to these systems. In this way, a candidate scalar product was identified and a large number of Macdonald superpolynomials were constructed. The correctness of the construction was corroborated by various conjectural properties that provide natural extensions to superspace of classical results on Macdonald polynomials. However, establishing the existence of the Macdonald superpolynomials remained an open problem, the corresponding eigenvalue problem being still missing.

The existence issue is resolved here: it is demonstrated that the superspace extension of the criteria (1) has a solution. This is our main result (the notation that follows is explained in full detail in Section 2).

Theorem 1. Given a superpartition $\Lambda=\left(\Lambda^{a} ; \Lambda^{s}\right)$ of fermionic degree $m$, there is a unique symmetric superpolynomial $P_{\Lambda}=P_{\Lambda}(x, \theta ; q, t)$, with $x=$ $\left(x_{1}, x_{2}, \ldots\right)$ and $\theta=\left(\theta_{1}, \theta_{2}, \ldots\right)$, such that:

1) $P_{\Lambda}=m_{\Lambda}+$ lower terms,

2) $\left\langle\left\langle P_{\Lambda} \mid P_{\Omega}\right\rangle\right\rangle_{q, t}=0$ if $\Lambda \neq \Omega$,

where $m_{\Lambda}$ is a monomial superpolynomial and where lower terms refer to the dominance ordering on superpartitions (see (10)). The scalar product is defined by

$$
\left\langle\left\langle p_{\Lambda} \mid p_{\Omega}\right\rangle\right\rangle_{q, t}=(-1)^{\left(\begin{array}{c}
m \\
2
\end{array}\right)} z_{\Lambda}(q, t) \delta_{\Lambda \Omega},
$$


where

$$
z_{\Lambda}(q, t)=z_{\Lambda^{s}} q^{\left|\Lambda^{a}\right|} \prod_{i=1}^{\ell\left(\Lambda^{s}\right)} \frac{1-q^{\Lambda_{i}^{s}}}{1-t^{\Lambda_{i}^{s}}}
$$

\subsection{A key relationship: The connection with Macdonald non-symmetric polynomials}

The existence proof proceeds indirectly, via an alternative definition of the Macdonald superpolynomials, this one relying on a suitable (anti)symmetrization of the non-symmetric Macdonald polynomials. Such a construction is akin to that of the Jack superpolynomials in terms of the non-symmetric polynomials worked out in [10, Sect. 9]. However, the present construction turns out to be trickier than in the Jack case. Indeed, Macdonald polynomials with prescribed symmetry - a priori, expected building blocks - are obtained by $t$-(anti)symmetrization of some subset of variables, where the role of symmetric group is played by the Hecke algebra [1,3]. However, anticommuting variable cannot be $t$-antisymmetrized, and the construction has to incorporate both the usual antisymmetrization and the $t$-symmetrization, the two operations being applied to distinct set of variables. This is made explicit in eq. (33).

This definition of the Macdonald superpolynomials in terms of nonsymmetric Macdonald polynomials is a crucial step toward the characterization of the former in terms of an eigenvalue problem. Recall that these non-symmetric polynomials are eigenfunctions of the Cherednik operators [8]. The mere symmetrization procedure that defines the Macdonald superpolynomials indicates how to dress symmetric combinations of the Cherednik operators in order to generate operators whose eigenfunctions are the Macdonald superpolynomials. Because we have two sets of variables (commuting and anticommuting), we need two families of commuting operators to obtain a non-degenerate eigenvalue characterization (see e.g. [10] for the Jack case). We thereby construct two generating functions of commuting operators. These are the natural extension of the Sekiguchi operators characterizing the Jack superpolynomials and introduced in [14, Sect. 3]. To fully characterize the eigenvalue problem, it is sufficient to consider the simplest representative of each family.

The proof of the existence of the superpolynomials defined by the two conditions in Theorem 1 proceeds along standard arguments. But a crucial and difficult result that needs to be established is the self-adjoint property 
of the two eigenoperators. The outline of this proof was provided to us by Alain Lascoux [22].

The relationship between Macdonald superpolynomials and the nonsymmetric polynomials has a further direct consequence: it implies a second orthogonality relation, where in this case, the scalar product is a constantterm expression. We show that when the number of variables tends to infinity, the norm calculated from the constant-term scalar product is equal (up to a $q$-power) to the norm, conjectured in [7], with respect to the scalar product defined in Theorem 1.

\subsection{Organization of the article}

The outline of the article is the following. In Section 2, we recall some basic definitions related to superpolynomials and superpartitions. Section 3 is also devoted to a review of known results, here pertaining to non-symmetric Macdonald polynomials. The definition of the Macdonald superpolynomials in terms of non-symmetric Macdonald polynomials is presented in Section 4.1. In Section 4.2, we introduce two generating functions for commuting operators and show that they have the Macdonald superpolynomials as their common eigenfunctions. This eigenfunction characterization allows us to demonstrate in Section 4.3 that the Macdonald superpolynomials have a triangular decomposition in the monomial basis. In preparation for the orthogonality proof, the eigenvalue problem defining the Macdonald superpolynomials is simplified in Section 4.4, where it is shown to be sufficient to consider two eigenoperators to get a non-degenerate characterization. The proof of the orthogonality with respect to the scalar product (4), which solves the existence issue in Theorem 1, is worked out in Section 5. At first, the problem is reformulated in terms of action of the two eigenoperators on the kernel. The long proof of the self-adjoint property of the Macdonald-type operators is worked out in Section 5.2. As a natural extension of these orthogonality results, a non-trivial duality relation is established in Section 5.3. Given this, all the tools for the demonstration of the evaluation and norm conjectures of [7] are available, proof that would follow that in [13] for the Jack superpolynomials. Finally, a second orthogonality relation is demonstrated in Section 6 . The corresponding norm is evaluated, and shown to be equal, up to a power of $q$, to the conjectural norm of the Macdonald superpolynomials with respect to the original scalar product. The combinatorial identity on which this claim relies is demonstrated in the Appendix.

The last section (Section 7), devoted to the generalized Kostka coefficients, is somewhat off the streamline of this article but should be viewed 
in the context of the continuation of [7]. The superspace version of the Macdonald positivity conjecture is recalled in Section 7.1. Two symmetry properties of the generalized Kostka coefficients are presented. Although these are rather direct extensions of the usual symmetry relations given in [24], one of these involve a new combinatorial number that is specific to superpartitions. This new data illustrates well the kind of novelties brought in by the introduction of anticommuting variables and the richness of the combinatorics of superpartitions. Tables of Kostka coefficients are appended to this section.

Two new conjectures concerning the Kostka coefficients are given in Section 7.2. Both results exhibit a different relationship between the $(q, t)$ Kostka coefficients in the $m=1$ sector with the ordinary (i.e., $m=0$ ) $(q, t)$-Kostka coefficients. In particular, Conjecture 25 expresses the usual $(q, t)$-Kostka coefficients for partitions of degree $n$ as a sum of $(q, t)$-Kostka coefficients for superpartitions of degree $n-1$ and $m=1$. In other words, the super-version of the Macdonald positivity conjecture provides a refinement of the usual ones. Let us digress briefly and point out that this result naturally poses the question: to which extent could we get information on the usual $(q, t)$-Kostka coefficients from the perspective of the Macdonald superpolynomials? We intend to investigate this point elsewhere from a particular angle. Such a connexion, at this stage, could rightly be called "Science fiction and Macdonald's superpolynomials" [5].

\section{Symmetric polynomials in superspace}

A polynomial in superspace, or equivalently, a superpolynomial, is a polynomial in the usual $N$ variables $x_{1}, \ldots, x_{N}$ and the $N$ anticommuting variables $\theta_{1}, \ldots, \theta_{N}$ over a certain field, which will be taken throughout this article to be $\mathbb{Q}(q, t)$. A superpolynomial $P(x, \theta)$, with $x=\left(x_{1}, \ldots, x_{N}\right)$ and $\theta=\left(\theta_{1}, \ldots, \theta_{N}\right)$, is said to be symmetric if the following is satisfied [9]:

$$
\mathcal{K}_{\sigma} P(x, \theta)=P(x, \theta) \quad \text { for all } \sigma \in S_{N},
$$

where

$$
\mathcal{K}_{\sigma}=\kappa_{\sigma} K_{\sigma}, \quad \text { with } \quad\left\{\begin{array}{l}
K_{\sigma}:\left(x_{1}, \ldots, x_{N}\right) \mapsto\left(x_{\sigma(1)}, \ldots, x_{\sigma(N)}\right) \\
\kappa_{\sigma}:\left(\theta_{1}, \ldots, \theta_{N}\right) \mapsto\left(\theta_{\sigma(1)}, \ldots, \theta_{\sigma(N)}\right) .
\end{array}\right.
$$

The space of symmetric superpolynomials in $N$ variables over the field $\mathbb{Q}(q, t)$ will be denoted $\mathscr{R}_{N}$, and its inverse limit by $\mathscr{R}$ (loosely speaking, the number of variables is considered infinite in $\mathscr{R}$ ). 
Before defining superpartitions, we recall some definitions related to partitions [24]. A partition $\lambda=\left(\lambda_{1}, \lambda_{2}, \ldots\right)$ of degree $|\lambda|$ is a vector of non-negative integers such that $\lambda_{i} \geq \lambda_{i+1}$ for $i=1,2, \ldots$ and such that $\sum_{i} \lambda_{i}=|\lambda|$. The length $\ell(\lambda)$ of $\lambda$ is the number of non-zero entries of $\lambda$. Each partition $\lambda$ has an associated Ferrers diagram with $\lambda_{i}$ lattice squares in the $i^{\text {th }}$ row, from the top to bottom. Any lattice square in the Ferrers diagram is called a cell (or simply a square), where the cell $(i, j)$ is in the $i$ th row and $j$ th column of the diagram. The conjugate $\lambda^{\prime}$ of a partition $\lambda$ is represented by the diagram obtained by reflecting $\lambda$ about the main diagonal. Given a cell $s=(i, j)$ in $\lambda$, we let

$$
a_{\lambda}(s)=\lambda_{i}-j, \quad \text { and } \quad l_{\lambda}(s)=\lambda_{j}^{\prime}-i .
$$

The quantities $a_{\lambda}(s)$ and $l_{\lambda}(s)$ are respectively called the arm-length and leg-length. We say that the diagram $\mu$ is contained in $\lambda$, denoted $\mu \subseteq \lambda$, if $\mu_{i} \leq \lambda_{i}$ for all $i$. Finally, $\lambda / \mu$ is a horizontal (resp. vertical) $n$-strip if $\mu \subseteq \lambda$, $|\lambda|-|\mu|=n$, and the skew diagram $\lambda / \mu$ does not have two cells in the same column (resp. row).

Symmetric superpolynomials are naturally indexed by superpartitions [9]. A superpartition $\Lambda$ of degree $(n \mid m)$ and length $\ell$ is a pair $\left(\Lambda^{\circledast}, \Lambda^{*}\right)$ of partitions $\Lambda^{\circledast}$ and $\Lambda^{*}$ such that [13]:

1. $\Lambda^{*} \subseteq \Lambda^{\circledast}$;

2. the degree of $\Lambda^{*}$ is $n$;

3. the length of $\Lambda^{\circledast}$ is $\ell$;

4. the skew diagram $\Lambda^{\circledast} / \Lambda^{*}$ is both a horizontal and a vertical $m$-strip. ${ }^{1}$

We refer to $m$ and $n$ respectively as the fermionic degree and total degree of $\Lambda$. Obviously, if $\Lambda^{\circledast}=\Lambda^{*}=\lambda$, then $\Lambda=(\lambda, \lambda)$ can be interpreted as the partition $\lambda$.

We will also need another characterization of a superpartition. A superpartition $\Lambda$ is a pair of partitions $\left(\Lambda^{a} ; \Lambda^{s}\right)=\left(\Lambda_{1}, \ldots, \Lambda_{m} ; \Lambda_{m+1}, \ldots, \Lambda_{\ell}\right)$, where $\Lambda^{a}$ is a partition with $m$ distinct parts (one of them possibly equal to zero), and $\Lambda^{s}$ is an ordinary partition. The correspondence between $\left(\Lambda^{\circledast}, \Lambda^{*}\right)$ and $\left(\Lambda^{a} ; \Lambda^{s}\right)$ is given explicitly as follows: given $\left(\Lambda^{\circledast}, \Lambda^{*}\right)$, the parts of $\Lambda^{a}$ correspond to the parts of $\Lambda^{*}$ such that $\Lambda_{i}^{\circledast} \neq \Lambda_{i}^{*}$, while the parts of $\Lambda^{s}$ correspond to the parts of $\Lambda^{*}$ such that $\Lambda_{i}^{\circledast}=\Lambda_{i}^{*}$.

The conjugate of a superpartition $\Lambda=\left(\Lambda^{\circledast}, \Lambda^{*}\right)$ is $\Lambda^{\prime}=\left(\left(\Lambda^{\circledast}\right)^{\prime},\left(\Lambda^{*}\right)^{\prime}\right)$. A diagrammatic representation of $\Lambda$ is given by the Ferrers diagram of $\Lambda^{*}$ with circles added in the cells corresponding to $\Lambda^{\circledast} / \Lambda^{*}$. For instance, if

\footnotetext{
${ }^{1}$ Some authors call such a diagram an $m$-rook strip.
} 
$\Lambda=\left(\Lambda^{a} ; \Lambda^{s}\right)=(3,1,0 ; 2,1)$, we have $\Lambda^{\circledast}=(4,2,2,1,1)$ and $\Lambda^{*}=(3,2,1,1)$, so that

(9)

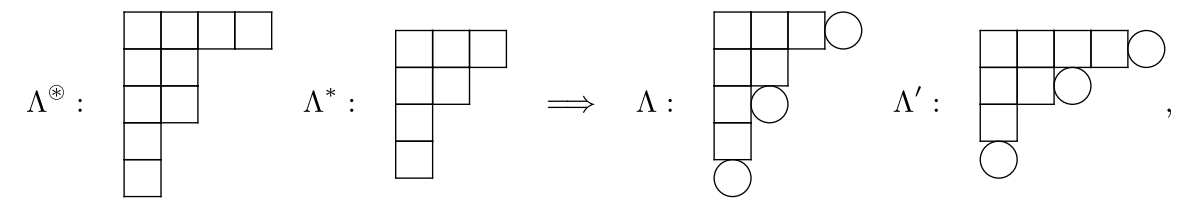

where the last diagram illustrates the conjugation operation that corresponds, as usual, to replacing rows by columns.

The extension of the dominance ordering to superpartitions is [13]:

$$
\Omega \leq \Lambda \quad \text { iff } \quad \operatorname{deg}(\Lambda)=\operatorname{deg}(\Omega), \quad \Omega^{*} \leq \Lambda^{*} \quad \text { and } \quad \Omega^{\circledast} \leq \Lambda^{\circledast}
$$

Note that comparing two superpartitions amounts to comparing two pairs of ordinary partitions, $\left(\Omega^{*}, \Lambda^{*}\right)$ and $\left(\Omega^{\circledast}, \Lambda^{\circledast}\right)$, with respect to the usual dominance ordering:

$$
\mu \leq \lambda \quad \text { iff } \quad|\mu|=|\lambda| \quad \text { and } \quad \mu_{1}+\cdots+\mu_{i} \leq \lambda_{1}+\cdots+\lambda_{i} \quad \forall i .
$$

Two simple bases of the space of symmetric polynomials in superspace (with commuting indeterminates $x_{1}, \ldots, x_{N}$ and anticommuting inderterminates $\left.\theta_{1}, \ldots, \theta_{N}\right)$ will be particularly relevant to our work:

1. the extension of the monomial symmetric functions, $m_{\Lambda}=m_{\Lambda}(x, \theta)$, defined by

$$
m_{\Lambda}=\sum_{\sigma \in S_{N}}{ }^{\prime} \mathcal{K}_{\sigma}\left(\theta_{1} \cdots \theta_{m} x_{1}^{\Lambda_{1}} \cdots x_{\ell}^{\Lambda_{\ell}}\right)
$$

where the sum is over the permutations of $\{1, \ldots, N\}$ that produce distinct terms;

2. the generalization of the power-sum symmetric functions, $p_{\Lambda}=p_{\Lambda}(x, \theta)$, defined by

$$
p_{\Lambda}=\tilde{p}_{\Lambda_{1}} \cdots \tilde{p}_{\Lambda_{m}} p_{\Lambda_{m+1}} \cdots p_{\Lambda_{\ell}}
$$

where

$$
\tilde{p}_{k}=\sum_{i=1}^{N} \theta_{i} x_{i}^{k} \quad \text { and } \quad p_{r}=\sum_{i=1}^{N} x_{i}^{r},
$$

with $k \geq 0$ and $r \geq 1$. 


\section{The non-symmetric Macdonald polynomials}

The ordinary Macdonald polynomials can be defined by the conditions (1) and (2) in (1). But they could alternatively be defined directly in terms of the so-called non-symmetric Macdonald polynomials by a suitable symmetrization process $[25,8]$ (see also [26, 27]). As will be shown in the following section, this can also be done for their superspace extension. But since this result uses a fair amount of notations and definitions, it is convenient to summarize these here.

The non-symmetric Macdonald polynomials are defined in terms of an eigenvalue problem formulated in terms of the Cherednik operators [8]. They are constructed from the operators $T_{i}$ defined as

$$
T_{i}=t+\frac{t x_{i}-x_{i+1}}{x_{i}-x_{i+1}}\left(K_{i, i+1}-1\right), \quad i=1, \ldots, N-1,
$$

and

$$
T_{0}=t+\frac{q t x_{N}-x_{1}}{q x_{N}-x_{1}}\left(K_{1, N} \tau_{1} \tau_{N}^{-1}-1\right),
$$

where we recall that $K_{i, j}$ exchanges the variables $x_{i}$ and $x_{j}$. Note that for $t=1, T_{i}$ reduces to $K_{i, i+1}$. The $T_{i}$ 's satisfy the affine Hecke algebra relations $(0 \leq i \leq N-1)$ :

$$
\begin{aligned}
& \left(T_{i}-t\right)\left(T_{i}+1\right)=0 \\
& T_{i} T_{i+1} T_{i}=T_{i+1} T_{i} T_{i+1} \\
& T_{i} T_{j}=T_{j} T_{i}, \quad i-j \neq \pm 1 \quad \bmod N
\end{aligned}
$$

where the indices are taken modulo $N$. To define the Cherednik operators, we also need to introduce the $q$-shift operators

$$
\tau_{i}:\left\{\begin{array}{l}
x_{i} \mapsto q x_{i}, \\
x_{j} \mapsto x_{j} \text { if } j \neq i,
\end{array}\right.
$$

and the operator $\omega$ defined as:

$$
\omega=K_{N-1, N} \cdots K_{1,2} \tau_{1} .
$$

We note that $\omega T_{i}=T_{i-1} \omega$ for $i=2, \ldots, N-1$. 
We are now in position to define the Cherednick operators:

$$
Y_{i}=t^{-N+i} T_{i} \cdots T_{N-1} \omega T_{1}^{-1} \cdots T_{i-1}^{-1},
$$

where $T_{j}^{-1}$ (also denoted $\bar{T}_{j}$ below) is

$$
T_{j}^{-1}=t^{-1}-1+t^{-1} T_{j}
$$

which follows from the quadratic relation (17) of the Hecke algebra. These operators satisfy the following relations $[8,18]$ :

$$
\begin{aligned}
T_{i} Y_{i} & =Y_{i+1} T_{i}+(t-1) Y_{i} \\
T_{i} Y_{i+1} & =Y_{i} T_{i}-(t-1) Y_{i} \\
T_{i} Y_{j} & =Y_{j} T_{i} \quad \text { if } j \neq i, i+1 .
\end{aligned}
$$

It can be easily deduced from these relations that

$$
\left(Y_{i}+Y_{i+1}\right) T_{i}=T_{i}\left(Y_{i}+Y_{i+1}\right) \quad \text { and } \quad\left(Y_{i} Y_{i+1}\right) T_{i}=T_{i}\left(Y_{i} Y_{i+1}\right)
$$

But more importantly, the $Y_{i}$ 's commute among each others, $\left[Y_{i}, Y_{j}\right]=0$, and can therefore be simultaneously diagonalized. Their eigenfunctions are the (monic) non-symmetric Macdonald polynomials (labeled by compositions). To be more precise, the non-symmetric Macdonald polynomial $E_{\eta}$ is the unique polynomial with rational coefficients in $q$ and $t$ that is triangularly related to the monomials (in the Bruhat ordering on compositions)

$$
E_{\eta}=x^{\eta}+\sum_{\nu \prec \eta} b_{\eta \nu} x^{\nu}
$$

and that satisfies, for all $i=1, \ldots, N$,

$$
Y_{i} E_{\eta}=\bar{\eta}_{i} E_{\eta}, \quad \text { where } \quad \bar{\eta}_{i}=q^{\eta_{i}} t^{-\bar{l}_{\eta}(i)}
$$

with $\bar{l}_{\eta}(i)=\#\left\{k<i \mid \eta_{k} \geq \eta_{i}\right\}+\#\left\{k>i \mid \eta_{k}>\eta_{i}\right\}$. The Bruhat order on compositions is defined as follows:

$$
\nu \prec \eta \quad \text { iff } \quad \nu^{+}<\eta^{+} \quad \text { or } \quad \nu^{+}=\eta^{+} \quad \text { and } \quad w_{\eta}<w_{\nu} \text {, }
$$

where $\eta^{+}$is the partition associated to $\eta$ and $w_{\eta}$ is the unique permutation of minimal length such that $\eta=w_{\eta} \eta^{+}\left(w_{\eta}\right.$ permutes the entries of $\left.\eta^{+}\right)$. In 
the Bruhat order on the symmetric group, $w_{\eta}<w_{\nu}$ iff $w_{\eta}$ can be obtained as a proper subword of $w_{\nu}$.

The following two properties of the non-symmetric Macdonald polynomials will be needed below. The first one expresses the stability of the polynomials $E_{\eta}$ with respect to the number of variables (see e.g. [27, eq. (3.2)]):

$$
E_{\eta}\left(x_{1}, \ldots, x_{N-1}, 0\right)= \begin{cases}E_{\eta_{-}}\left(x_{1}, \ldots, x_{N-1}\right) & \text { if } \eta_{N}=0 \\ 0 & \text { if } \eta_{N} \neq 0\end{cases}
$$

where $\eta_{-}=\left(\eta_{1}, \ldots, \eta_{N-1}\right)$. The second one gives the action of the operators $T_{i}$ on $E_{\eta}$ (see e.g. [3, eq. (8)] and [2]):

$$
T_{i} E_{\eta}= \begin{cases}\left(\frac{t-1}{1-\delta_{i, \eta}^{-1}}\right) E_{\eta}+t E_{s_{i} \eta} & \text { if } \eta_{i}<\eta_{i+1} \\ t E_{\eta} & \text { if } \eta_{i}=\eta_{i+1} \\ \left(\frac{t-1}{1-\delta_{i, \eta}^{-1}}\right) E_{\eta}+\frac{\left(1-t \delta_{i, \eta}\right)\left(1-t^{-1} \delta_{i, \eta}\right)}{\left(1-\delta_{i, \eta}\right)^{2}} E_{s_{i} \eta} & \text { if } \eta_{i}>\eta_{i+1}\end{cases}
$$

where $\delta_{i, \eta}=\bar{\eta}_{i} / \bar{\eta}_{i+1}$ and $s_{i} \eta=\left(\eta_{1}, \ldots, \eta_{i-1}, \eta_{i+1}, \eta_{i}, \eta_{i+2}, \ldots, \eta_{N}\right)$.

Finally, we introduce the $t$-symmetrization and $t$-antisymmetrization operators of variables $x_{1}, \ldots, x_{N} \quad[25]$ :

$$
U_{N}^{+}=\sum_{\sigma \in S_{N}} T_{\sigma} \quad \text { and } \quad U_{N}^{-}=\sum_{\sigma \in S_{N}}(-t)^{-\ell(\sigma)} T_{\sigma}
$$

where

$$
T_{\sigma}=T_{i_{1}} \cdots T_{i_{\ell}} \quad \text { if } \quad \sigma=s_{i_{1}} \cdots s_{i_{\ell}} .
$$

Note that for any polynomial $f$ in the variables $x_{1}, \ldots, x_{N}$, we have $K_{i, i+1} U_{N}^{+} f=U_{N}^{+} f$, but $K_{i, i+1} U_{N}^{-} f \neq-U_{N}^{-} f$ since [27, eq.(2.26)]

$$
U_{N}^{-} f=t^{-\left(\begin{array}{c}
N \\
2
\end{array}\right)} \frac{\Delta_{N}^{t}}{\Delta_{N}} A_{N} f
$$

where

$$
A_{N}=\sum_{\sigma \in S_{N}}(-1)^{\ell(\sigma)} K_{\sigma}, \quad \Delta_{N}^{t}=\prod_{1 \leq i<j \leq N}\left(t x_{i}-x_{j}\right), \quad \Delta_{N}=\Delta_{N}^{1} .
$$

Note that $A_{N}$ is the usual antisymmetrization operator. Below, we will designate by $S_{m}$ and $S_{m^{c}}$ the group of permutations of the variables $x_{1}, \ldots, x_{m}$ 
and $x_{m+1}, \ldots, x_{N}$ respectively. For instance, $U_{m}^{-}$and $U_{m^{c}}^{+}$are defined as in (29) but with $S_{N}$ replaced by $S_{m}$ and $S_{m^{c}}$ respectively. Similarly, we will frequently use the notation $\Delta_{m}^{t}$ which is defined as in (32) but with $N$ replaced by $m$.

\section{Macdonald superpolynomials}

\subsection{Definition of the Macdonald superpolynomials}

We are now in position to define the Macdonald superpolynomials in terms of the non-symmetric Macdonald polynomials. We will prove later that the Macdonald superpolynomials defined in the next definition do in fact provide a solution to the existence problem in Theorem 1.

Definition 2. The Macdonald superpolynomials $P_{\Lambda}=P_{\Lambda}(x, \theta ; q, t)$ are defined as

$$
P_{\Lambda}=\frac{(-1)^{\left(\begin{array}{c}
m \\
2
\end{array}\right)}}{f_{\Lambda^{s}}(t) t^{\operatorname{inv}\left(\Lambda^{s}\right)}} \sum_{\sigma \in S_{N} /\left(S_{m} \times S_{m^{c}}\right)} \mathcal{K}_{\sigma} \theta_{1} \cdots \theta_{m} A_{m} U_{m^{c}}^{+} E_{\Lambda^{R}},
$$

where

$$
f_{\Lambda^{s}}(t)=\prod_{j \geq 0}\left[n_{\Lambda^{s}}(j)\right]_{t} !
$$

with $n_{\Lambda^{s}}(j)$ being the number of occurrences of $j$ in $\Lambda^{s}$ and $\Lambda^{R}$ stands for the concatenation of $\Lambda^{a}$ and $\Lambda^{s}$ read in reverse order:

$$
\Lambda^{R}=\left(\Lambda_{m}, \ldots, \Lambda_{1}, \Lambda_{N}, \ldots, \Lambda_{m+1}\right)
$$

In (33), we extended the usual concept of inversion on a permutation to a partition: $\operatorname{inv}\left(\Lambda^{s}\right)$ is the number of inversions in $\Lambda^{s}$, the latter number being equal to

$$
\operatorname{inv}(\lambda)=\#\left\{n \geq i>j \mid \lambda_{i}<\lambda_{j}\right\}
$$

where $n$ is the number of entries in $\lambda$ (including 0's). For instance, we have $\operatorname{inv}(22100)=8$. In $(34)$, we also used the following standard notation:

$$
[k]_{t} !=[1]_{t}[2]_{t} \cdots[k]_{t} \quad \text { with } \quad[m]_{t}=\left(1-t^{m}\right) /(1-t) .
$$


We first show that the stability of $E_{\eta}$ with respect to the number of variables can be lifted to that of $P_{\Lambda}$.

Proposition 3. Suppose that $N>m$. Then the Macdonald superpolynomials $P_{\Lambda}$ are stable with respect the number of variables, that is,

$$
\begin{aligned}
& P_{\Lambda}\left(x_{1}, \ldots, x_{N-1}, 0, \theta_{1}, \ldots, \theta_{N-1}, 0\right) \\
& \quad= \begin{cases}P_{\Lambda_{-}}\left(x_{1}, \ldots, x_{N-1}, \theta_{1}, \ldots, \theta_{N-1}\right) & \text { if } \Lambda_{N}=0 \\
0 & \text { if } \Lambda_{N} \neq 0\end{cases}
\end{aligned}
$$

where $\Lambda_{-}=\left(\Lambda_{1}, \ldots, \Lambda_{m} ; \Lambda_{m+1}, \ldots, \Lambda_{N-1}\right)$.

Proof. From the definition of $P_{\Lambda}$ it is immediate that it suffices to show that

$$
\left[U_{m^{c}}^{+} E_{\Lambda^{R}}\right]_{x_{N}=0}= \begin{cases}\frac{c_{\Lambda_{-}}}{c_{\Lambda}} U_{m_{-}^{c}}^{+} E_{\Lambda_{-}^{R}} & \text { if } \Lambda_{N}=0, \\ 0 & \text { if } \Lambda_{N} \neq 0,\end{cases}
$$

where $m_{-}^{c}$ refers to the reduced set of variables $x_{m+1}, \ldots, x_{N-1}$ and

$$
\Lambda_{-}^{R}=\left(\Lambda_{-}\right)^{R}=\left(\Lambda_{m}, \ldots, \Lambda_{1}, \Lambda_{N-1}, \ldots, \Lambda_{m+1}\right) .
$$

We stress that although $\Lambda_{-}$is a superpartition, $\Lambda_{-}^{R}$ is a composition. The constant $c_{\Lambda}(t)$ is the normalization constant in (33):

$$
c_{\Lambda}(t)=\frac{(-1)^{\left(\begin{array}{c}
m \\
2
\end{array}\right)}}{f_{\Lambda^{s}}(t) t^{\operatorname{inv}\left(\Lambda^{s}\right)}} .
$$

Now $U_{m^{c}}^{+}$can be factorized as follows:

$$
U_{m^{c}}^{+}=U_{m_{-}^{c}}^{+}\left(1+T_{N-1}+T_{N-1} T_{N-2}+\cdots+T_{N-1} \cdots T_{m+1}\right),
$$

so that

$$
\begin{aligned}
& {\left[U_{m^{c}}^{+} E_{\Lambda^{R}}\right]_{x_{N}=0}} \\
& \quad=U_{m_{-}^{c}}^{+}\left[\left(1+T_{N-1}+T_{N-1} T_{N-2}+\cdots+T_{N-1} \cdots T_{m+1}\right) E_{\Lambda^{R}}\right]_{x_{N}=0}
\end{aligned}
$$

Now, if $\Lambda^{s}$ has exactly $k=n_{\Lambda^{s}}(0)$ zero entries, it is easy to see from (27) and (28) that 


$$
\left[T_{N-1} \cdots T_{m+i} E_{\Lambda^{R}}\right]_{x_{N}=0}= \begin{cases}t^{N-m-i} E_{\Lambda_{-}^{R}} & \text { if } i \leq k \\ 0 & \text { if } i>k\end{cases}
$$

Hence

$$
\left[U_{m^{c}}^{+} E_{\Lambda^{R}}\right]_{x_{N}=0}= \begin{cases}t^{N-m-k}[k]_{t} U_{m_{-}^{c}}^{+} E_{\Lambda_{-}^{R}} & \text { if } k>0 \\ 0 & \text { if } k=0\end{cases}
$$

If $k>0$, we have that $\operatorname{inv}\left(\Lambda_{-}^{s}\right)=\operatorname{inv}\left(\Lambda^{s}\right)-N+m+k$. Therefore, $t^{N-m-k}[k]_{t}=$ $c_{\Lambda_{-}} / c_{\Lambda}$ and the proposition follows.

\subsection{Two families of commuting (eigen)operators}

We now introduce two families of operators generalizing those introduced for the Jack superpolynomials [14, Sect. 3] and defined as

$$
D^{*}(u ; q, t)=\sum_{m=0}^{N} \sum_{\sigma \in S_{N} /\left(S_{m} \times S_{m^{c}}\right)} \mathcal{K}_{\sigma}\left(\frac{\Delta_{m}}{\Delta_{m}^{t}} \prod_{i=1}^{N}\left(1+u Y_{i}\right) \frac{\Delta_{m}^{t}}{\Delta_{m}} \pi_{1, \ldots, m}\right)
$$

and

$$
\begin{aligned}
& D^{\circledast}(u ; q, t) \\
& \quad=\sum_{m=0}^{N} \sum_{\sigma \in S_{N} /\left(S_{m} \times S_{m^{c}}\right)} \mathcal{K}_{\sigma}\left(\frac{\Delta_{m}}{\Delta_{m}^{t}} \prod_{i=1}^{m}\left(1+u q Y_{i}\right) \prod_{i=m+1}^{N}\left(1+u Y_{i}\right) \frac{\Delta_{m}^{t}}{\Delta_{m}} \pi_{1, \ldots, m}\right) .
\end{aligned}
$$

The operator $\pi_{1, \ldots, m}$ is the projection operator defined as

$$
\pi_{1, \ldots, m}=\prod_{i=1}^{m} \theta_{i} \partial_{\theta_{i}} \prod_{j=m+1}^{N} \partial_{\theta_{j}} \theta_{j}
$$

In this equation, $\partial_{\theta_{i}}$ denotes the standard derivative with respect to the Grassmann variable $\theta_{i}$, which is a linear operator such that, for all polynomials $f=f(x, \theta)$ and $i, j \in\{1, \ldots, N\}$,

$$
\partial_{\theta_{i}}\left(x_{j} f\right)=x_{j} \partial_{\theta_{i}}(f) \quad \partial_{\theta_{i}}\left(\theta_{j} f\right)=\delta_{i, j} f-\theta_{j} \partial_{\theta_{i}}(f),
$$


and

$$
\partial_{\theta_{i}} \partial_{\theta_{j}}(f)=-\partial_{\theta_{j}} \partial_{\theta_{i}}(f) \quad \Longrightarrow \quad \partial_{\theta_{i}}^{2}(f)=0
$$

It is easy to see that

$$
\pi_{1, \ldots, m} \theta_{i_{1}} \cdots \theta_{i_{k}}= \begin{cases}\theta_{1} \cdots \theta_{m} & \text { if }\left\{i_{1}, \ldots, i_{k}\right\}=\{1, \ldots, m\} \\ 0 & \text { if }\left\{i_{1}, \ldots, i_{k}\right\} \neq\{1, \ldots, m\}\end{cases}
$$

If we let $D_{n}^{*}$ (resp. $D_{n}^{\circledast}$ ) be the coefficient of $u^{n}$ in $D^{*}(u ; q, t)$ (resp. $\left.D^{\circledast}(u ; q, t)\right)$, the operators $D^{*}(u ; q, t)$ and $D^{\circledast}(u ; q, t)$ can be seen as the generating series of the operators $D_{n}^{*}$ and $D_{n}^{\circledast}$ respectively. These operators, when restricted to act on $\mathscr{R}_{N}$, can be considered as the generalization to superspace of the Macdonald operators.

As will be shown below, the superpolynomials $P_{\Lambda}$ are eigenfunctions of both $D^{*}(u ; q, t)$ and $D^{\circledast}(u ; q, t)$. A rationale for the rather intricate structure of the operators is the following: in order for $P_{\Lambda}$, as given by $(33)$, to be an eigenfunction of an operator built out of $\prod\left(1+u Y_{i}\right)$, the factor $\Delta_{m}^{t} / \Delta_{m}$ needs to be inserted to the right to transform $A_{m}$ into $U^{-}$via (31), so that $U^{-}$can be commuted through the factors $\prod\left(1+u Y_{i}\right)$. Finally, the term $\Delta_{m} / \Delta_{m}^{t}$ is added to the left to retransform $U^{-}$into $A_{m}$. We now state the eigenfunction characterization and plunge into the details of the proof.

Proposition 4. We have

$$
D^{*}(u ; q, t) P_{\Lambda}=\varepsilon_{\Lambda^{*}}(u ; q, t) P_{\Lambda} \quad \text { and } \quad D^{\circledast}(u ; q, t) P_{\Lambda}=\varepsilon_{\Lambda^{\circledast}}(u ; q, t) P_{\Lambda}
$$

where $\varepsilon_{\lambda}(u ; q, t)$ is given, for any partition $\lambda$, by

$$
\varepsilon_{\lambda}(u ; q, t)=\prod_{i=1}^{N}\left(1+u q^{\lambda_{i}} t^{1-i}\right)
$$

Proof. First observe that

$$
\pi_{1, \ldots, l} \mathcal{K}_{w} \theta_{1} \cdots \theta_{m}= \begin{cases}\mathcal{K}_{w} \theta_{1} \cdots \theta_{m} & \text { if } l=m \text { and } w \in S_{m} \times S_{m^{c}} \\ 0 & \text { otherwise }\end{cases}
$$


We thus have

$$
\begin{aligned}
& D^{*}(u ; q, t) P_{\Lambda} \\
& \quad=c_{\Lambda}(t) \sum_{\sigma \in S_{N} /\left(S_{m} \times S_{m^{c}}\right)} \mathcal{K}_{\sigma} \frac{\Delta_{m}}{\Delta_{m}^{t}} \prod_{i=1}^{N}\left(1+u Y_{i}\right) \frac{\Delta_{m}^{t}}{\Delta_{m}} \theta_{1} \cdots \theta_{m} A_{m} U_{m^{c}}^{+} E_{\Lambda^{R}} \\
& =c_{\Lambda}(t) \sum_{\sigma \in S_{N} /\left(S_{m} \times S_{m^{c}}\right)} \mathcal{K}_{\sigma} t^{\left(\begin{array}{c}
m \\
2
\end{array}\right)} \frac{\Delta_{m}}{\Delta_{m}^{t}} \prod_{i=1}^{N}\left(1+u Y_{i}\right) \theta_{1} \cdots \theta_{m} U_{m}^{-} U_{m^{c}}^{+} E_{\Lambda^{R}} .
\end{aligned}
$$

Relations (23) imply that the product $\prod_{i=1}^{N}\left(1+u Y_{i}\right)$ can be moved beyond the factors $U$ :

$$
\begin{aligned}
& D^{*}(u ; q, t) P_{\Lambda} \\
& \quad=c_{\Lambda}(t) \sum_{\sigma \in S_{N} /\left(S_{m} \times S_{m^{c}}\right)} \mathcal{K}_{\sigma} t^{\left(\begin{array}{c}
m \\
2
\end{array}\right)} \frac{\Delta_{m}}{\Delta_{m}^{t}} \theta_{1} \cdots \theta_{m} U_{m}^{-} U_{m^{c}}^{+} \prod_{i=1}^{N}\left(1+u Y_{i}\right) E_{\Lambda^{R}} \\
& \quad=c_{\Lambda}(t) \sum_{\sigma \in S_{N} /\left(S_{m} \times S_{m^{c}}\right)} \mathcal{K}_{\sigma} \theta_{1} \cdots \theta_{m} A_{m} U_{m^{c}}^{+} \prod_{i=1}^{N}\left(1+u Y_{i}\right) E_{\Lambda^{R}} .
\end{aligned}
$$

To prove the first statement, it thus suffices to prove that

$$
\left(\prod_{i=1}^{N}\left(1+u Y_{i}\right)\right) E_{\Lambda^{R}}=\varepsilon_{\Lambda^{*}}(u ; q, t) E_{\Lambda^{R}}
$$

Similarly, to prove the second statement, we have to prove that

$$
\left(\prod_{i=1}^{m}\left(1+u q Y_{i}\right) \prod_{i=m+1}^{N}\left(1+u Y_{i}\right)\right) E_{\Lambda^{R}}=\varepsilon_{\Lambda^{\circledast}}(u ; q, t) E_{\Lambda^{R}} .
$$

Let $\eta=\Lambda^{R}$ and suppose that $\eta_{i}=r$. It is easy to see that the quantity $\bar{l}_{\eta}(i)$ in the eigenvalue $\bar{\eta}_{i}=q^{r} t^{-\bar{l}_{\eta}(i)}$ of $Y_{i}$ (see $\left.(25)\right)$ is such that

$$
\begin{aligned}
\bar{l}_{\eta}(i)= & \#\left\{\text { rows of } \Lambda^{*} \text { of size larger than } r\right\} \\
& +\#\left\{\text { rows of } \Lambda^{R} \text { of size } r \text { above row } i\right\} .
\end{aligned}
$$


Therefore, letting

$$
\begin{aligned}
j_{i}= & \#\left\{\text { rows of } \Lambda^{*} \text { of size larger than } r\right\} \\
& +\#\left\{\text { rows of } \Lambda^{R} \text { of size } r \text { above row } i\right\}+1
\end{aligned}
$$

we have $\left\{j_{1}, \ldots, j_{N}\right\}=\{1, \ldots, N\}, \Lambda_{j_{i}}^{*}=r$ and we recover (56) in the form

$$
\left(\prod_{i=1}^{N}\left(1+u Y_{i}\right)\right) E_{\Lambda^{R}}=\prod_{j_{i}=1}^{N}\left(1+u q^{\Lambda_{j_{i}}^{*}} t^{1-j_{i}}\right) E_{\Lambda^{R}}
$$

Continuing with the same notation for the second case (57), we have that if $i$ belongs to $\{1, \ldots, m\}$ then $\eta_{i}=r$ is the highest row of size $r$ in $\eta$, and thus by (59) $\Lambda_{j_{i}}^{*}$ is also the highest row of size $r$ in $\Lambda^{*}$. Hence, the eigenvalue in this case is

$$
q Y_{i} E_{\Lambda^{R}}=q^{\Lambda_{j_{i}}^{*}+1} t^{1-j_{i}} E_{\Lambda^{R}}
$$

But since $i \in\{1, \ldots, m\}, \Lambda_{j_{i}}^{*} \in \Lambda^{a}$ and therefore $\Lambda_{j_{i}}^{*}+1=\Lambda_{j_{i}}^{\circledast}$. Now, if $i$ does not belong to $\{1, \ldots, m\}$, then we have $\Lambda_{j_{i}}^{*}=\Lambda_{j_{i}}^{\circledast}$ and then

$$
\left(\prod_{i=1}^{m}\left(1+u q Y_{i}\right) \prod_{i=m+1}^{N}\left(1+u Y_{i}\right)\right) E_{\Lambda^{R}}=\prod_{j_{i}=1}^{N}\left(1+u q^{\Lambda_{j_{i}}^{\circledast}} t^{1-j_{i}}\right) E_{\Lambda^{R}} .
$$

\subsection{The triangular decomposition of the Macdonald superpolynomials}

At this point, we have established that the $P_{\Lambda}$ 's are eigenfunctions of the operators $D^{*}(u ; q, t)$ and $D^{\circledast}(u ; q, t)$. We now show that this characterization of the Macdonald superpolynomials entails a triangular decomposition into monomials. The chosen normalization in (33) will make this decomposition unitriangular.

It is well known that if $x^{\gamma}$ appears in the monomial expansion of $Y_{i} x^{\eta}$, then $\gamma \leq \eta[25$, Eq. (4.13)]. This statement however is not sufficient for our purposes. We now give a slightly more precise characterization of the triangular action of the Cherednik operators $Y_{i}$ on monomials. For this, we need to define some operations on compositions. Suppose $i<j$. Let

$$
s_{i, j}\left(\ldots, \eta_{i}, \ldots, \eta_{j}, \ldots\right)=\left(\ldots, \eta_{j}, \ldots, \eta_{i}, \ldots\right) .
$$


Let also $\hat{s}_{i, j}$ be the following restriction of $s_{i, j}$ :

$$
\hat{s}_{i, j}\left(\ldots, \eta_{i}, \ldots, \eta_{j}, \ldots\right)=\left(\ldots, \eta_{j}, \ldots, \eta_{i}, \ldots\right) \text { only if } \eta_{i}>\eta_{j}
$$

Finally, for $\ell=1, \ldots,\left|\eta_{i}-\eta_{j}\right|-1$, let

$$
s_{i, j}^{(\ell)}\left(\ldots, \eta_{i}, \ldots, \eta_{j}, \ldots\right)= \begin{cases}\left(\ldots, \eta_{i}-\ell, \ldots, \eta_{j}+\ell, \ldots\right) & \text { if } \eta_{i}>\eta_{j} \\ \left(\ldots, \eta_{i}+\ell, \ldots, \eta_{j}-\ell, \ldots\right) & \text { if } \eta_{i}<\eta_{j}\end{cases}
$$

Note that we will often use $s_{i}, \hat{s}_{i}$, and $s_{i}^{(\ell)}$ instead of $s_{i, i+1}, \hat{s}_{i, i+1}$ and $s_{i, i+1}^{(\ell)}$, respectively.

Lemma 5. Suppose that $x^{\gamma}$ occurs in the monomial expansion of $Y_{i} x^{\eta}$. Then

$$
\gamma=g_{1} \cdots g_{r}(\eta)
$$

where $g_{k}$ either stands for $\hat{s}_{i_{k} j_{k}}$ or $s_{i_{k} j_{k}}^{\left(\ell_{k}\right)}$. We stress that if $g_{k}=\hat{s}_{i_{k} j_{k}}$ then $\omega_{i_{k}}>\omega_{j_{k}}$, where $\omega=g_{k+1} \cdots g_{r}(\eta)$.

Proof. One easily shows that

$$
T_{i} x^{\eta}=a_{\eta} x^{\eta}+b_{\eta} x^{s_{i} \eta}+(1-t) \operatorname{sgn}\left(\eta_{i}-\eta_{i+1}\right) \sum_{\ell=1}^{\left|\eta_{i}-\eta_{i+1}\right|-1} x^{s_{i}^{(\ell)} \eta},
$$

where $\operatorname{sgn}(x)$ is the sign of $x$ and

$$
a_{\eta}=\left\{\begin{array}{ll}
t-1, & \eta_{i}<\eta_{i+1}, \\
0, & \eta_{i} \geq \eta_{i+1},
\end{array} \quad \text { and } \quad b_{\eta}= \begin{cases}t, & \eta_{i} \leq \eta_{i+1} \\
1, & \eta_{i}>\eta_{i+1}\end{cases}\right.
$$

Thus, the monomials that appear in $T_{i} x^{\eta}$ are of the form $x^{s_{i}(\eta)}, x^{s_{i}^{(\ell)}(\eta)}$, for $\ell=1, \ldots,\left|\eta_{i}-\eta_{i+1}\right|-1$ and, possibly an extra $x^{\eta}$. The latter extra term $x^{\eta}$ appears only if $\eta_{i}<\eta_{i+1}$. We stress that if $\eta_{i}=\eta_{i+1}$, the operator $s_{i}$ also acts as the identity on $\eta$. However, even in the latter case, we prefer to write $s_{i} \eta$ explicitly in order to avoid any confusion with the identity operator that produces the very first term on the rhs of (67).

The action of the inverse operator $\bar{T}_{i}=t^{-1} T_{i}+\left(t^{-1}-1\right)$ immediately follows from (67). Once more, the monomials that appear in $\bar{T}_{i} x^{\eta}$ are of the form $x^{s_{i}(\eta)}, x^{s_{i}^{(\ell)}(\eta)}$, for $\ell=1, \ldots,\left|\eta_{i}-\eta_{i+1}\right|-1$ and, possibly an extra $x^{\eta}$. However, the latter extra term $x^{\eta}$ appears this time only if $\eta_{i}>\eta_{i+1}$. 
Hence, from the definition of $Y_{i}$, the terms $x^{\gamma}$ appearing in $Y_{i} x^{\eta}$ are such that

$$
\gamma=f_{i} \cdots f_{N-1} s_{N-1} \cdots s_{1} \bar{f}_{1} \cdots \bar{f}_{i-1}(\eta),
$$

where $f_{j}$ and $\bar{f}_{j}$ correspond either to $s_{j}, s_{j}^{(\ell)}$ or the identity (whenever allowed).

Let $j$ be such that $\bar{f}_{j}$ does not act as $s_{j}$, and suppose that $j$ is the rightmost amongst such terms. We have that (note that $j<i$ )

$$
\begin{aligned}
\gamma= & f_{i} \cdots f_{N-1} s_{N-1} \cdots s_{1} \bar{f}_{1} \\
& \cdots \bar{f}_{j-1} s_{j} \cdots s_{i-1}\left(s_{i-1} \cdots s_{j+1} s_{j} \bar{f}_{j} s_{j+1} \cdots s_{i-1}(\eta)\right) .
\end{aligned}
$$

Observe that $f_{i} \cdots f_{N-1} s_{N-1} \cdots s_{1} \bar{f}_{1} \cdots \bar{f}_{j-1} s_{j} \cdots s_{i-1}$ corresponds to the operator that appears in (69) with $\bar{f}_{k}=s_{k}$ for $k=j, \ldots, i-1$. If $\bar{f}_{j}$ is the identity, we have

$$
\begin{aligned}
& s_{i-1} \cdots s_{j+1} s_{j} \bar{f}_{j} s_{j+1} \cdots s_{i-1}(\eta) \\
& \quad=s_{i-1} \cdots s_{j+1} s_{j} s_{j+1} \cdots s_{i-1}(\eta)=s_{j, i}(\eta) .
\end{aligned}
$$

Note that by supposition, $\eta_{j}>\eta_{i}$ since $\bar{f}_{j}$ acted as the identity. If $\bar{f}_{j}=s_{j}^{(\ell)}$, we have

$$
\begin{aligned}
& s_{i-1} \cdots s_{j+1} s_{j} \bar{f}_{j} s_{j+1} \cdots s_{i-1}(\eta) \\
& \quad=s_{i-1} \cdots s_{j+1} s_{j} s_{j}^{(\ell)} s_{j+1} \cdots s_{i-1}(\eta)=s_{j, i}^{\left(\ell^{\prime}\right)}(\eta),
\end{aligned}
$$

for some $\ell^{\prime}$ (the $\ell^{\prime}$ is such that $s_{j, i} s_{j, i}^{(\ell)}=s_{j, i}^{\left(\ell^{\prime}\right)}$ ). Repeating the same process, we can get rid of all $\bar{f}_{k}$ such that $\bar{f}_{k}$ does not act as $s_{k}$. More explicitly, we have shown that

$$
\gamma=f_{i} \cdots f_{N-1} s_{N-1} \cdots s_{1} \bar{f}_{1} \cdots \bar{f}_{i-1}(\mu),
$$

where all the $\bar{f}_{k}$ act as $s_{k}$ and where $\mu$ is the composition obtained from $\eta$ by the action of some $s_{j i}^{\ell}$ and $\hat{s}_{j i}$ with $j<i$.

Now suppose that $f_{j}$ acts as the identity and suppose that $j$ is the rightmost such terms. In this case, (supposing that all $\bar{f}_{k}$ act as $s_{k}$ ) (73) becomes (note that $i<j$ this time)

$$
\begin{aligned}
\gamma & =f_{i} \cdots f_{j-1} f_{j} s_{j+1} \cdots s_{N-1} s_{N-1} \cdots s_{1} s_{1} \cdots s_{i-1}(\mu) \\
& =f_{i} \cdots f_{j-1} f_{j} s_{j} \cdots s_{i}(\mu) .
\end{aligned}
$$


Hence,

$$
\gamma=f_{i} \cdots f_{j-1} s_{j} s_{j} \cdots s_{i}\left(s_{i} s_{i+1} \cdots s_{j-1} f_{j} s_{j} s_{j-1} \cdots s_{i+1} s_{i}(\mu)\right) .
$$

If $f_{j}$ is the identity, we have

$$
\begin{aligned}
& \left(s_{i} s_{i+1} \cdots s_{j-1} f_{j} s_{j} s_{j-1} \cdots s_{i+1} s_{i}(\mu)\right) \\
& \quad=\left(s_{i} s_{i+1} \cdots s_{j-1} s_{j} s_{j-1} \cdots s_{i+1} s_{i}(\mu)\right)=s_{i, j}(\mu) .
\end{aligned}
$$

By supposition, $\mu_{i}>\mu_{j}$ since $f_{j}$ acted as the identity, so $s_{i, j}(\mu)=\hat{s}_{i, j}(\mu)$. If $f_{j}=s_{j}^{(\ell)}$, then for some $\ell^{\prime}$,

$$
\begin{aligned}
\gamma & =f_{i} \cdots f_{j-1} s_{j} s_{j} \cdots s_{i}\left(s_{i} s_{i+1} \cdots s_{j-1} s_{j}^{(\ell)} s_{j} s_{j-1} \cdots s_{i+1} s_{i}(\mu)\right) \\
& =s_{i, j}^{\left(\ell^{\prime}\right)}(\mu) .
\end{aligned}
$$

Applying these operations again and again we obtain that

$$
\gamma=f_{i} \cdots f_{N-1} s_{N-1} \cdots s_{1} \bar{f}_{1} \cdots \bar{f}_{i-1}\left(g_{1} \cdots g_{r}(\eta)\right)
$$

where the $g_{k}$ 's are such as specified in the statement of the lemma, and where all the $f_{k}$ 's and $\bar{f}_{k}$ 's act as $s_{k}$. But replacing $f_{k}=\bar{f}_{k}=s_{k}$ in the previous equation, we obtain that

$$
\gamma=g_{1} \cdots g_{r}(\eta)
$$

which completes the proof of the lemma.

Proposition 6. We have

$$
D^{*}(u ; q, t) m_{\Lambda}=\varepsilon_{\Lambda^{*}}(u ; q, t) m_{\Lambda}+\sum_{\Omega<\Lambda} v_{\Lambda \Omega} m_{\Omega}
$$

and, similarly,

$$
D^{\circledast}(u ; q, t) m_{\Lambda}=\varepsilon_{\Lambda \circledast}(u ; q, t) m_{\Lambda}+\sum_{\Omega<\Lambda} \bar{v}_{\Lambda \Omega} m_{\Omega} .
$$

Proof. Let $m$ be a fixed integer. Define $\eta^{\circledast}$ to be equal to $\left(\eta+1^{m}\right)^{+}$, and define $\eta^{*}$ to be equal to $\eta^{+}$. We say that a row $i$ of $\eta^{\circledast}$ is fermionic if $\eta_{i}^{\circledast} \neq \eta_{i}^{*}$ and bosonic otherwise. 
We will first show that if $x^{\gamma}$ appears in $Y_{i} x^{\eta}$, then $\gamma^{*} \leq \eta^{*}$ and $\gamma^{\circledast} \leq \eta^{\circledast}$. It is immediate from the definition of the Bruhat order on compositions and the fact that $Y_{i}$ acts triangularly on monomials that $\gamma^{*} \leq \eta^{*}$. We have left to show that $\gamma^{\circledast} \leq \eta^{\circledast}$. From Lemma 5, we only need to show that if $\gamma=g_{k}(\eta)$, for $g_{k}$ such as specified in the statement of the lemma, then $\gamma^{\circledast} \leq \eta^{\circledast}$. Suppose that $g_{k}=s_{i, j}$. From Lemma 5 , we have $\eta_{i}>\eta_{j}$. The only non-trivial case is when $i \leq m$ and $j>m$. In that case, it is easy to see that $\gamma^{\circledast}$ will be obtained from $\eta^{\circledast}$ by interchanging a fermionic element and a bosonic one, with the fermionic one being the largest. This is easily seen to imply that $\gamma^{\circledast} \leq \eta^{\circledast}$. Now suppose that $g_{k}=s_{i, j}^{(\ell)}$. Again the only non-trivial case is when $i \leq m$ and $j>m$. In that case, given that $\ell<\left(\eta_{i}-\eta_{j}\right), \gamma^{\circledast}$ is obtained from $\eta^{\circledast}$ by modifying a fermionic element and a bosonic one in such a way that none of the two modified rows is larger than the largest of the original ones. It is then immediate that again $\gamma^{\circledast} \leq \eta^{\circledast}$.

Suppose now that $\Lambda$ is a superpartition in the fermionic sector $m$ (we will consider that $\Lambda$ is also the composition $\left.\left(\Lambda_{1}^{a}, \ldots, \Lambda_{m}^{a}, \Lambda_{1}^{s}, \ldots\right)\right)$, so that the monomial $m_{\Lambda}$ can be written as

$$
m_{\Lambda}=\frac{1}{f_{\Lambda^{s}}(1)} \sum_{\sigma \in S_{N} /\left(S_{m} \times S_{m} c\right)} \mathcal{K}_{\sigma}\left(\theta_{1} \cdots \theta_{m} A_{m} \sum_{w \in S_{m_{c}}} x^{w(\Lambda)}\right) .
$$

Then,

$$
\begin{aligned}
& D^{*}(u ; q, t) m_{\Lambda} \\
& \quad \propto \sum_{\sigma \in S_{N} /\left(S_{m} \times S_{m^{c}}\right)} \mathcal{K}_{\sigma} \frac{\Delta_{m}}{\Delta_{m}^{t}} \prod_{i=1}^{N}\left(1+u Y_{i}\right) \frac{\Delta_{m}^{t}}{\Delta_{m}} \theta_{1} \cdots \theta_{m} A_{m} \sum_{w \in S_{m^{c}}} x^{w(\Lambda)} \\
& =\sum_{\sigma \in S_{N} /\left(S_{m} \times S_{m^{c}}\right)} \mathcal{K}_{\sigma} t^{\left(\begin{array}{c}
m \\
2
\end{array}\right)} \frac{\Delta_{m}}{\Delta_{m}^{t}} \prod_{i=1}^{N}\left(1+u Y_{i}\right) \theta_{1} \cdots \theta_{m} U_{m}^{-} \sum_{w \in S_{m^{c}}} x^{w(\Lambda)} \\
& \propto \sum_{\sigma \in S_{N}} \mathcal{K}_{\sigma} \theta_{1} \cdots \theta_{m} A_{m} \sum_{w \in S_{m} c} \prod_{i=1}^{N}\left(1+u Y_{i}\right) x^{w(\Lambda)} .
\end{aligned}
$$

Letting $\eta=w(\Lambda)$ we have that $\eta^{*}=\Lambda^{*}$ and $\eta^{\circledast}=\Lambda^{\circledast}$. From our prior analysis, we thus have that all the terms $x^{\gamma}$ that appear in $\prod_{i=1}^{N}\left(1+u Y_{i}\right) x^{w(\Lambda)}$ are such that $\gamma^{*} \leq \Lambda^{*}$ and $\gamma^{\circledast} \leq \Lambda^{\circledast}$. The triangularity of the action of $D^{*}(u ; q, t)$ is then immediate. The triangularity of the action of $D^{\circledast}(u ; q, t)$ 
is proven in the same manner. Finally, proceeding as in the proof of Proposition 4 , it can be checked that

$$
\prod_{i=1}^{N}\left(1+u Y_{i}\right) x^{\Lambda}=\varepsilon_{\Lambda^{*}}(u ; q, t) x^{\Lambda}+\text { lower terms }
$$

and similarly, that

$$
\prod_{i=1}^{m}\left(1+u q Y_{i}\right) \prod_{i=m+1}^{N}\left(1+u Y_{i}\right) x^{\Lambda}=\varepsilon_{\Lambda \circledast}(u ; q, t) x^{\Lambda}+\text { lower terms. }
$$

This completes the proof of the proposition.

Proposition 7. The Macdonald superpolynomials are unitriangularly related to the monomials. In other words,

$$
P_{\Lambda}=m_{\Lambda}+\sum_{\Omega<\Lambda} c_{\Lambda \Omega}(q, t) m_{\Omega}
$$

where we observe that $c_{\Lambda \Omega}(q, t)$ does not depend on $N$ from Proposition 3.

Proof. The triangularity is almost immediate from Propositions 4 and 6 . Suppose that there exists a term $m_{\Omega}$ such that $\Omega \not \leq \Lambda$ in $P_{\Lambda}$ and suppose that $\Omega$ is maximal among those superpartitions. Then by Proposition 6 the coefficient of $m_{\Omega}$ in $D^{*}(u ; q, t) P_{\Lambda}$ and $D^{\circledast}(u ; q, t) P_{\Lambda}$ is respectively equal to $c_{\Lambda \Omega} \varepsilon_{\Omega^{*}}(u ; q, t)$ and $c_{\Lambda \Omega} \varepsilon_{\Omega^{\circledast}}(u ; q, t)$. Since we cannot have $\varepsilon_{\Omega^{*}}(u ; q, t)=$ $\varepsilon_{\Lambda^{*}}(u ; q, t)$ and $\varepsilon_{\Omega^{\circledast}}(u ; q, t)=\varepsilon_{\Lambda^{\circledast}}(u ; q, t)$ at the same time $\left(\varepsilon_{\Lambda^{*}}(u ; q, t)\right.$ and $\varepsilon_{\Lambda \circledast}(u ; q, t)$ uniquely determine $\left.\Lambda\right)$, we have the contradiction that $P_{\Lambda}$ is not an eigenfuntion of $D^{*}(u ; q, t)$ and $D^{\circledast}(u ; q, t)$ with eigenvalues $\varepsilon_{\Lambda^{*}}(u ; q, t)$ and $\varepsilon_{\Lambda \circledast}(u ; q, t)$ respectively.

We now have to prove that the coefficient of $m_{\Lambda}$ in $P_{\Lambda}$ is equal to 1 . To prove this, we follow [27, Lemma 5.5]. We start with the expression (33) for $P_{\Lambda}$, written compactly as

$$
P_{\Lambda}=c_{\Lambda} \sum_{\sigma \in S_{N} /\left(S_{m} \times S_{m^{c}}\right)} \mathcal{K}_{\sigma} \theta_{1} \cdots \theta_{m} A_{m} U_{m^{c}}^{+} E_{\Lambda^{R}}
$$

where the constant $c_{\Lambda}$ given in (33) (or in (40)). It suffices to concentrate on the coefficient of the term in $x^{\Lambda}$. From (24), we see that it can only arise 
from the dominant term

(86)

$$
P_{\Lambda}=c_{\Lambda} \sum_{\sigma \in S_{N} /\left(S_{m} \times S_{m} c\right)} \mathcal{K}_{\sigma}\left(\theta_{1} \cdots \theta_{m} A_{m} \sum_{w \in S_{m^{c}}} T_{w} x^{\Lambda^{R}}\right)+\text { lower terms, }
$$

where it should be observed that $T_{w}$ acts on $x_{m+1}^{\Lambda_{N}} x_{m+2}^{\Lambda_{N-1}} \cdots x_{N}^{\Lambda_{m+1}}$. Let

$$
\underline{\eta}=\left(\Lambda_{1}, \ldots, \Lambda_{m}, \Lambda_{N}, \ldots, \Lambda_{m+1}\right),
$$

and write

$$
A_{m} x^{\Lambda^{R}}=(-1)^{\operatorname{inv}\left(\Lambda^{a}\right)} A_{m} x^{\underline{\eta}},
$$

where $\operatorname{inv}\left(\Lambda^{a}\right)=m(m-1) / 2$. We thus have

$$
P_{\Lambda}=(-1)^{\left(\begin{array}{c}
m \\
2
\end{array}\right)} c_{\Lambda} \sum_{\sigma \in S_{N} /\left(S_{m} \times S_{m^{c}}\right)} \mathcal{K}_{\sigma}\left(\theta_{1} \cdots \theta_{m} A_{m} \sum_{w \in S_{m^{c}}} T_{w} x \underline{\eta}\right)+\text { lower terms. }
$$

It is easy to show (from the explicit action of $T_{i}$ ) that [27, Lemma 2.3]

$$
T_{w} x^{\underline{\eta}}=d_{w(\underline{\eta})} x^{w(\underline{\eta})}+\sum_{\nu \prec w(\underline{\eta})} d_{\nu} x^{\nu}
$$

where $d_{\nu} \in \mathbb{Q}(t)$. In the following, we denote by $\left[x^{\Lambda}\right] F(x)$ the coefficient of $x^{\Lambda}$ in the expression $F(x)$. The coefficient of the term $x^{\Lambda}$ in $U_{m^{c}}^{+} x^{\underline{\eta}}$ is given by

$$
\left[x^{\Lambda}\right] \sum_{w \in S_{m^{c}}} T_{w} x^{\underline{\eta}}=\sum_{w \in S_{m c} \mid w(\underline{\eta})=\Lambda} t^{\ell(w)},
$$

where $\ell(w)$ is the length of the permutation $w$. Suppose that all the parts of $\Lambda^{s}$ are distinct. Then there is only one permutation $w$ that can give $w\left(\left(\Lambda^{s}\right)^{R}\right)=\Lambda^{s}$ and its length is given by $\ell(w)=\operatorname{inv}\left(\Lambda^{s}\right)$. Now, when there are repeated parts in $\Lambda^{s}, \operatorname{inv}\left(\Lambda^{s}\right)$ is the length of the permutation of minimal length such that $w\left(\left(\Lambda^{s}\right)^{R}\right)=\Lambda^{s}$. However, we must also consider the contributions resulting from permuting these repeated parts. So, in general we can write

$$
\left[x^{\Lambda}\right] \sum_{w \in S_{m^{c}}} T_{w} x^{\underline{\eta}}=t^{\operatorname{inv}\left(\Lambda^{s}\right)} \prod_{i=0}^{\Lambda_{1}^{s}}\left(\sum_{w^{(i)} \in S_{m(i)}} t^{\ell\left(w^{(i)}\right)}\right),
$$


where $m(i)=n_{\Lambda^{s}}(i)$. Using $\sum_{\sigma \in S_{k}} t^{\ell(\sigma)}=[k]_{t}$ !, we then obtain

$$
\left[x^{\Lambda}\right] \sum_{w \in S_{m^{c}}} T_{w} x^{\underline{\eta}}=t^{\operatorname{inv}\left(\Lambda^{s}\right)} \prod_{i=0}^{\Lambda_{1}^{s}}\left[n_{\Lambda^{s}}(i)\right]_{t} !
$$

Now, since $U_{m^{c}}^{+} x \underline{\eta}$ is symmetric in the variables $x_{m+1}, \ldots, x_{N}$ and $A_{m} U_{m^{c}}^{+} x \underline{\eta}$ is antisymmetric in the variables $x_{1}, \ldots, x_{m}$, the monomial $m_{\Lambda}$ is reconstructed with these actions and multiplication by $\theta_{1} \cdots \theta_{m}$. Hence, we have

$$
\left[m_{\Lambda}\right] \sum_{\sigma \in S_{N} /\left(S_{m} \times S_{m^{c}}\right)} \mathcal{K}_{\sigma}\left(\theta_{1} \cdots \theta_{m} A_{m} U_{m^{c}}^{+} x \underline{\eta}\right)=t^{\operatorname{inv}\left(\Lambda^{s}\right)} \prod_{i=0}^{\Lambda_{1}^{s}}\left[n_{\Lambda^{s}}(i)\right]_{t} !,
$$

which immediately gives

$$
\begin{aligned}
{\left[m_{\Lambda}\right] P_{\Lambda} } & =(-1)^{\left(\begin{array}{c}
m \\
2
\end{array}\right)} c_{\Lambda} t^{\operatorname{inv}\left(\Lambda^{s}\right)} \prod_{i=0}^{\Lambda_{1}^{s}}\left[n_{\Lambda^{s}}(i)\right]_{t} ! \\
& =(-1)^{\left(\begin{array}{c}
m \\
2
\end{array}\right)} c_{\Lambda} t^{\operatorname{inv}\left(\Lambda^{s}\right)} f_{\Lambda^{s}}(t)=1 .
\end{aligned}
$$

Corollary 8. The $P_{\Lambda}$ 's form a basis of the space $\mathscr{R}_{N}$ of symmetric polynomials in superspace.

Recall that $D_{n}^{*}$ (resp. $\left.D_{n}^{\circledast}\right)$ is the coefficient of $u^{n}$ in $D^{*}(u ; q, t)$ (resp. $\left.D^{\circledast}(u ; q, t)\right)$.

Corollary 9. The $2 N$ operators $D_{n}^{*}$ and $D_{\ell}^{\circledast}$ for $n, \ell=1, \ldots, N$ are mutually commuting when their action is restricted to $\mathscr{R}_{N}$.

\subsection{A simplified eigenfunction characterization}

We end this section with a characterization of the $P_{\Lambda}$ 's as common eigenfuntions of two commuting operators. In the notation of Corollary 9, we have

$$
\begin{aligned}
D_{1}^{*}= & \sum_{m=0}^{N} \sum_{\sigma \in S_{N} /\left(S_{m} \times S_{m^{c}}\right)} \mathcal{K}_{\sigma}\left(\frac{\Delta_{m}}{\Delta_{m}^{t}}\left(Y_{1}+\cdots+Y_{N}\right) \frac{\Delta_{m}^{t}}{\Delta_{m}} \pi_{1, \ldots, m}\right), \\
D_{1}^{\circledast}= & \sum_{m=0}^{N} \sum_{\sigma \in S_{N} /\left(S_{m} \times S_{m^{c}}\right)} \mathcal{K}_{\sigma}\left(\frac { \Delta _ { m } } { \Delta _ { m } ^ { t } } \left(q Y_{1}+\cdots+q Y_{m}\right.\right. \\
& \left.\left.+Y_{m+1}+\cdots+Y_{N}\right) \frac{\Delta_{m}^{t}}{\Delta_{m}} \pi_{1, \ldots, m}\right) .
\end{aligned}
$$


From the linear term in $u$ in (52), we see that the eigenvalue of the above operators on $P_{\Lambda}$ are

$$
D_{1}^{\circ} P_{\Lambda}=\varepsilon_{\Lambda^{\circ}}^{(1)} P_{\Lambda}, \quad \text { where } \circ \in\{*, \circledast\}, \quad \text { and } \quad \varepsilon_{\lambda}^{(1)}=\sum_{i} q^{\lambda_{i}} t^{1-i} .
$$

Given these two operators, it is natural to consider the following differences:

$$
\begin{aligned}
\mathcal{O}_{1} & =\frac{1}{1-q}\left(D_{1}^{*}-D_{1}^{\circledast}\right) \\
& =\sum_{m=0}^{N} \sum_{\sigma \in S_{N} /\left(S_{m} \times S_{m^{c}}\right)} \mathcal{K}_{\sigma}\left(\frac{\Delta_{m}}{\Delta_{m}^{t}}\left(Y_{1}+\cdots+Y_{m}\right) \frac{\Delta_{m}^{t}}{\Delta_{m}} \pi_{1, \ldots, m}\right), \\
\mathcal{O}_{2} & =\frac{1}{q-1}\left(q D_{1}^{*}-D_{1}^{\circledast}\right) \\
& =\sum_{m=0}^{N} \sum_{\sigma \in S_{N} /\left(S_{m} \times S_{m} c\right.} \mathcal{K}_{\sigma}\left(\frac{\Delta_{m}}{\Delta_{m}^{t}}\left(Y_{m+1}+\cdots+Y_{N}\right) \frac{\Delta_{m}^{t}}{\Delta_{m}} \pi_{1, \ldots, m}\right) .
\end{aligned}
$$

From (97), we get

$$
\mathcal{O}_{1} P_{\Lambda}=\frac{1}{q-1}\left[\sum_{i=1}^{N}\left(q^{\Lambda_{i}^{\circledast}}-q^{\Lambda_{i}^{*}}\right) t^{1-i}\right] P_{\Lambda}
$$

and

$$
\mathcal{O}_{2} P_{\Lambda}=\frac{1}{q-1}\left[\sum_{i=1}^{N}\left(q^{\Lambda_{i}^{*}+1}-q^{\Lambda_{i}^{\circledast}}\right) t^{1-i}\right] P_{\Lambda} .
$$

Observe that the two eigenvalues are in one-to-one correspondence with $\Lambda$. We also define

$$
\overline{\mathcal{O}}_{1}=\sum_{m=0}^{N} \sum_{\sigma \in S_{N} /\left(S_{m} \times S_{m c}\right)} \mathcal{K}_{\sigma}\left(\frac{\Delta_{m}}{\Delta_{m}^{t}}\left(\bar{Y}_{1}+\cdots+\bar{Y}_{m}\right) \frac{\Delta_{m}^{t}}{\Delta_{m}} \pi_{1, \ldots, m}\right),
$$

where $\bar{Y}_{i}$ is the inverse of $Y_{i}$ :

$$
\bar{Y}_{i}=t^{N-i} T_{i-1} \cdots T_{1} \bar{\omega} \bar{T}_{N-1} \cdots \bar{T}_{i},
$$


with $\bar{T}_{j}=T_{j}^{-1}$ and $\bar{\omega}=\omega^{-1}$. We have

$$
\overline{\mathcal{O}}_{1} P_{\Lambda}=\frac{1}{1 / q-1}\left[\sum_{i=1}^{N}\left(q^{-\Lambda_{i}^{\circledast}}-q^{-\Lambda_{i}^{*}}\right) t^{i-1}\right] P_{\Lambda}
$$

Finally, we define $E_{1, N}=\overline{\mathcal{O}}_{1}$ and $E_{2, N}=\mathcal{O}_{2}-\sum_{i=1}^{N} t^{1-i}$. It is easy to see that

$$
\begin{aligned}
& E_{1, N} P_{\Lambda}=\left(\sum_{i: \Lambda_{i}^{\circledast} \neq \Lambda_{i}^{*}} q^{-\Lambda_{i}^{*}} t^{i-1}\right) P_{\Lambda} \text { and } \\
& E_{2, N} P_{\Lambda}=\left(\sum_{i: \Lambda_{i}^{\circledast}=\Lambda_{i}^{*}}\left(q^{\Lambda_{i}^{*}}-1\right) t^{1-i}\right) P_{\Lambda} .
\end{aligned}
$$

Thus, the eigenvalues of $E_{1, N}$ and $E_{2, N}$ do not depend on $N$. This property explains the substraction of $\sum_{i=1}^{N} t^{1-i}$ in the definition of $E_{2, N}$ : it ensures that the eigenvalue does not depend upon the zeros in $\Lambda^{s}$. This, and the fact that $P_{\Lambda}$ is stable with respect to the number of variables, allows us to define

$$
E_{1}=\lim _{\longleftarrow} E_{1, N} \quad \text { and } \quad E_{2}=\lim _{\longleftarrow} E_{2, N} .
$$

We have the following characterization of $P_{\Lambda}$.

Proposition 10. The polynomial $P_{\Lambda}$ is the unique polynomial in $\mathscr{R}$ such that

1. $P_{\Lambda}=m_{\Lambda}+\sum_{\Omega<\Lambda} v_{\Lambda \Omega} m_{\Omega}$

2. $E_{1} P_{\Lambda}=\left(\sum_{\substack{i \\ \Lambda_{i}^{\circledast} \neq \Lambda_{i}^{*}}} q^{-\Lambda_{i}^{*}} t^{i-1}\right) P_{\Lambda} \quad$ and $\quad E_{2} P_{\Lambda}=\left(\sum_{\substack{i \\ \Lambda_{i}^{\circledast}=\Lambda_{i}^{*}}}\left(q^{\Lambda_{i}^{*}}-1\right) t^{1-i}\right) P_{\Lambda}$.

Proof. From Proposition 7 and (105), the superpolynomial $P_{\Lambda}$ satisfies the two properties. Since $E_{1}$ and $E_{2}$ have together distinct eigenvalues, the two properties characterize $P_{\Lambda}$.

\section{Orthogonality and existence}

\subsection{Kernel and orthogonality}

Let $x=\left(x_{1}, x_{2}, \ldots\right)$ and $y=\left(y_{1}, y_{2}, \ldots\right)$ be two sets of commuting variables, and let $\theta=\left(\theta_{1}, \theta_{2}, \ldots\right)$ and $\phi=\left(\phi_{1}, \phi_{2}, \ldots\right)$ be two sets of anticommuting 
variables. We define the following reproducing kernel [7]:

$$
K(x, \theta ; y, \phi)=\prod_{i, j} \frac{\left(t x_{i} y_{j} ; q\right)_{\infty}}{\left(x_{i} y_{j} ; q\right)_{\infty}}\left(1+\frac{\theta_{i} \phi_{j}}{1-q^{-1} x_{i} y_{j}}\right)
$$

where

$$
(a ; q)_{\infty}=\prod_{k=0}^{\infty}\left(1-a q^{k}\right)
$$

Observe that

$$
K(x, \theta ; y, \phi)=K^{0}(x ; y) \prod_{i, j}\left(1+\frac{\theta_{i} \phi_{j}}{1-q^{-1} x_{i} y_{j}}\right),
$$

where

$$
K^{0}(x ; y)=\prod_{i, j} \frac{\left(t x_{i} y_{j} ; q\right)_{\infty}}{\left(x_{i} y_{j} ; q\right)_{\infty}}
$$

is the usual Macdonald reproducing kernel [24, eq. VI.2.4]. It is straightforward to show that

$$
K(x, \theta ; y, \phi)=\sum_{\Lambda}(-1)^{\left(\begin{array}{c}
m \\
2
\end{array}\right)} z_{\Lambda}(q, t)^{-1} p_{\Lambda}(x, \theta) p_{\Lambda}(y, \phi),
$$

where $z_{\Lambda}(q, t)$ was defined in (4). Recall from Theorem 1 that the factor $(-1)^{\left(\begin{array}{c}m \\ 2\end{array}\right)} z_{\Lambda}(q, t)$ is the norm of the scalar product of the power sums, i.e.,

$$
\left\langle\left\langle p_{\Lambda} \mid p_{\Omega}\right\rangle\right\rangle_{q, t}=(-1)^{\left(\begin{array}{c}
m \\
2
\end{array}\right)} z_{\Lambda}(q, t) \delta_{\Lambda \Omega}
$$

The following propositions are standard and can be proven using methods similar to those found in Macdonald's book [24].

Proposition 11. For each $n, m$, let $\left\{u_{\Lambda}\right\}$ and $\left\{v_{\Lambda}\right\}$ be bases of $\mathscr{R}^{n, m}$, where $\mathscr{R}^{n, m}$ is the subspace of $\mathscr{R}$ of degree $(n \mid m)$. Then the following conditions are equivalent:

1. $\left\langle\left\langle u_{\Lambda} \mid v_{\Omega}\right\rangle\right\rangle_{q, t}=\delta_{\Lambda \Omega}$ for all $\Lambda, \Omega$;

2. $K(x, \theta ; y, \phi)=\sum_{\Lambda} u_{\Lambda}(x, \theta) v_{\Lambda}(y, \phi)$.

Proposition 12. Let $E: \mathscr{R} \rightarrow \mathscr{R}$ be a $\mathbb{Q}(q, t)$-linear operator. Then the following conditions are equivalent: 
1. $\langle\langle E f \mid g\rangle\rangle_{q, t}=\left\langle\langle f \mid E g\rangle_{q, t}\right.$ for all $f, g \in \mathscr{R}$;

2. $E^{(x, \theta)} K(x, \theta ; y, \phi)=E^{(y, \phi)} K(x, \theta ; y, \phi)$, where $E^{(x, \theta)}$ (resp. $\left.E^{(y, \phi)}\right)$ acts on the variables $x$ and $\theta$ (resp. $y$ and $\phi)$.

The rest of this section will be devoted to the proof of the following proposition, whose corollary implies Theorem 1.

Theorem 13. We have that

$$
\begin{aligned}
& E_{1}^{(x, \theta)} K(x, \theta ; y, \phi)=E_{1}^{(y, \phi)} K(x, \theta ; y, \phi), \\
& E_{2}^{(x, \theta)} K(x, \theta ; y, \phi)=E_{2}^{(y, \phi)} K(x, \theta ; y, \phi) .
\end{aligned}
$$

Corollary 14. The Macdonald superpolynomial $P_{\Lambda}$ is such that

1. $P_{\Lambda}=m_{\Lambda}+$ lower terms;

2. $\left\langle\left\langle P_{\Lambda} \mid P_{\Omega}\right\rangle\right\rangle_{q, t}=0$ if $\Lambda \neq \Omega$.

Proof. The triangularity was proven in Proposition 7. By Proposition 12 and Theorem 13 we have

$$
\left\langle\left\langle E_{1} P_{\Lambda} \mid P_{\Omega}\right\rangle\right\rangle_{q, t}=\left\langle\left\langle P_{\Lambda} \mid E_{1} P_{\Omega}\right\rangle\right\rangle_{q, t} \quad \text { and } \quad\left\langle\left\langle E_{2} P_{\Lambda} \mid P_{\Omega}\right\rangle\right\rangle_{q, t}=\left\langle\left\langle P_{\Lambda} \mid E_{2} P_{\Omega}\right\rangle\right\rangle_{q, t} .
$$

Given that the two operators have together distinct eigenvalues, this immediately gives

$$
\left\langle\left\langle P_{\Lambda} \mid P_{\Omega}\right\rangle\right\rangle_{q, t}=0 \quad \text { if } \quad \Lambda \neq \Omega
$$

\subsection{Self-adjointness of $E_{1}$ and $E_{2}$}

The proof of Theorem 13 is quite involved. It relies fundamentally on Proposition 16, whose proof was kindly outlined to us by Alain Lascoux [22]. Theorem 13 follows from the following proposition since $\overline{\mathcal{O}}_{1}=E_{1, N}$ and $\mathcal{O}_{2}$ differs from $E_{2, N}$ by a constant.

Proposition 15. Let $K_{N}(x, \theta ; y, \phi)$ be the restriction of $K(x, \theta ; y, \phi)$ to $N$ variables. We have

$$
\begin{aligned}
& \overline{\mathcal{O}}_{1}^{(x, \theta)} K_{N}(x, \theta ; y, \phi)=\overline{\mathcal{O}}_{1}^{(y, \phi)} K_{N}(x, \theta ; y, \phi), \\
& \mathcal{O}_{2}^{(x, \theta)} K_{N}(x, \theta ; y, \phi)=\mathcal{O}_{2}^{(y, \phi)} K_{N}(x, \theta ; y, \phi),
\end{aligned}
$$

where the superscripts indicate the variables on which the operators act. 
The first step in the proof of Proposition 15 will amount to reformulate the conditions (115) in a more tractable form (this is Proposition 16 below).

Firstly, it is not difficult to see that the coefficient of $\theta_{1} \cdots \theta_{m} \phi_{1} \cdots \phi_{m}$ in $K_{N}(x, \theta ; y, \phi)$ is equal to (up to a sign and a power of $q$ )

$$
\left[\theta_{1} \cdots \theta_{m} \phi_{1} \cdots \phi_{m}\right] K_{N}(x, \theta ; y, \phi) \propto \frac{K_{N}^{0}(x ; y) \Delta_{m}(x) \Delta_{m}(y)}{\prod_{1 \leq i, j \leq m}\left(1-q^{-1} x_{i} y_{j}\right)},
$$

where we recall that $\left[\theta_{1} \cdots \theta_{m} \phi_{1} \cdots \phi_{m}\right] f$ stands for the coefficient of the monomial $\theta_{1} \cdots \theta_{m} \phi_{1} \cdots \phi_{m}$ in $f$. This is seen as follows: up to a sign, the coefficient of $\theta_{1} \cdots \theta_{m} \phi_{1} \cdots \phi_{m}$ in $\prod_{i, j}\left[1+\theta_{i} \phi_{j}\left(1-x_{i} y_{j}\right)^{-1}\right]$ is

$$
\begin{aligned}
\sum_{\sigma \in S_{m}}(-1)^{\ell(\sigma)} \frac{1}{\prod_{i=1}^{m}\left(1-x_{i} y_{\sigma(i)}\right)} & =\sum_{\sigma}(-1)^{\ell(\sigma)} \sum_{\eta} x^{\eta} y^{\sigma(\eta)} \\
& =\sum_{\eta}(-1)^{\operatorname{sign}(\eta)} x^{\eta} s_{\eta^{+}-\delta^{(m)}}(y) \Delta(y),
\end{aligned}
$$

where $\eta$ is a composition with distinct parts (otherwise the result is zero by antisymmetry), $\eta^{+}$is the partition corresponding to $\eta$ and $\operatorname{sign}(\eta)$ is the sign of the permutation that changes $\eta$ to $\eta^{+}$. The second equality is obtained by interchanging the two summations and using the expression of the Schur polynomial $s_{\mu}$ as a ratio of two determinants (cf. [24, eq. (3.1)]). By splitting the sum over $\eta$ into a sum over $\eta^{+}$and a summation over permutations of $\eta^{+}$, and then by letting $\lambda=\eta^{+}-\delta^{(m)}$, we can rewrite (117) as

$$
\begin{aligned}
& \sum_{\sigma \in S_{m}}(-1)^{\ell(\sigma)} \frac{1}{\prod_{i=1}^{m}\left(1-x_{i} y_{\sigma(i)}\right)} \\
& \quad=\Delta_{m}(x) \Delta_{m}(y) \sum_{\lambda} s_{\lambda}(x) s_{\lambda}(y)=\frac{\Delta_{m}(x) \Delta_{m}(y)}{\prod_{1 \leq i, j \leq m}\left(1-x_{i} y_{j}\right)} .
\end{aligned}
$$

Note that [24, eq. (4.3)] was used for getting the last expression on the right-hand side. By substituting $x_{i} \rightarrow x_{i} / q$ and multiplying the result by $K_{N}^{0}(x ; y)$, we recover $(116)$.

More generally, the coefficient of $\theta_{1} \cdots \theta_{m} \phi_{j_{1}} \cdots \phi_{j_{m}}$ (with $j_{1}<\cdots<j_{m}$ ) in $K_{N}(x, \theta ; y, \phi)$ is

$$
\begin{aligned}
& {\left[\theta_{1} \cdots \theta_{m} \phi_{j_{1}} \cdots \phi_{j_{m}}\right] K_{N}(x, \theta ; y, \phi)} \\
& \quad \propto K_{1, j_{1}}^{(y)} \cdots K_{m, j_{m}}^{(y)} \frac{K_{N}^{0}(x ; y) \Delta_{m}(x) \Delta_{m}(y)}{\prod_{1 \leq i, j \leq m}\left(1-q^{-1} x_{i} y_{j}\right)} .
\end{aligned}
$$


Given this result, the coefficient of $\theta_{i_{1}} \cdots \theta_{i_{m}} \phi_{j_{1}} \cdots \phi_{j_{m}}$ in $\overline{\mathcal{O}}_{1}^{(x, \theta)} K_{N}(x, \theta ; y, \phi)$ is proportional to

$K_{1, i_{1}}^{(x)} \cdots K_{m, i_{m}}^{(x)} K_{1, j_{1}}^{(y)} \cdots K_{m, j_{m}}^{(y)} \frac{\Delta_{m}(x)}{\Delta_{m}^{t}(x)}\left(\bar{Y}_{1}^{(x)}+\cdots+\bar{Y}_{m}^{(x)}\right) \frac{K_{N}^{0}(x ; y) \Delta_{m}^{t}(x) \Delta_{m}(y)}{\prod_{1 \leq i, j \leq m}\left(1-q^{-1} x_{i} y_{j}\right)}$.

Similarly, the coefficient of $\theta_{i_{1}} \cdots \theta_{i_{m}} \phi_{j_{1}} \cdots \phi_{j_{m}}$ in $\overline{\mathcal{O}}_{1}^{(y, \phi)} K_{N}(x, \theta ; y, \phi)$ is proportional to (with the same proportionality factor as above)

$$
K_{1, i_{1}}^{(x)} \cdots K_{m, i_{m}}^{(x)} K_{1, j_{1}}^{(y)} \cdots K_{m, j_{m}}^{(y)} \frac{\Delta_{m}(y)}{\Delta_{m}^{t}(y)}\left(\bar{Y}_{1}^{(y)}+\cdots+\bar{Y}_{m}^{(y)}\right) \frac{K_{N}^{0}(x ; y) \Delta_{m}(x) \Delta_{m}^{t}(y)}{\prod_{1 \leq i, j \leq m}\left(1-q^{-1} x_{i} y_{j}\right)} .
$$

The first relation in (115) is equivalent to the equality of both coefficients. Canceling the permutation operators in the equality between (120) and (121) yields

$$
\begin{aligned}
& \frac{\Delta_{m}(x)}{\Delta_{m}^{t}(x)}\left(\bar{Y}_{1}^{(x)}+\cdots+\bar{Y}_{m}^{(x)}\right) \frac{K_{N}^{0}(x ; y) \Delta_{m}^{t}(x) \Delta_{m}(y)}{\prod_{1 \leq i, j \leq m}\left(1-q^{-1} x_{i} y_{j}\right)} \\
& \quad=\frac{\Delta_{m}(y)}{\Delta_{m}^{t}(y)}\left(\bar{Y}_{1}^{(y)}+\cdots+\bar{Y}_{m}^{(y)}\right) \frac{K_{N}^{0}(x ; y) \Delta_{m}(x) \Delta_{m}^{t}(y)}{\prod_{1 \leq i, j \leq m}\left(1-q^{-1} x_{i} y_{j}\right)}
\end{aligned}
$$

Proceeding similarly for $\mathcal{O}_{2}$, and using commutativity of the type $Y_{i}^{(x)} f(y)=$ $f(y) Y_{i}^{(x)}$, we obtain the two relations appearing in the following proposition, whose proof thus implies Proposition 15.

Proposition 16. We have

$$
\begin{aligned}
& \left(\bar{Y}_{1}^{(x)}+\cdots+\bar{Y}_{m}^{(x)}\right) \frac{K_{N}^{0}(x ; y) \Delta_{m}^{t}(x) \Delta_{m}^{t}(y)}{\prod_{1 \leq i, j \leq m}\left(1-q^{-1} x_{i} y_{j}\right)} \\
& \quad=\left(\bar{Y}_{1}^{(y)}+\cdots+\bar{Y}_{m}^{(y)}\right) \frac{K_{N}^{0}(x ; y) \Delta_{m}^{t}(x) \Delta_{m}^{t}(y)}{\prod_{1 \leq i, j \leq m}\left(1-q^{-1} x_{i} y_{j}\right)} \\
& \left(Y_{m+1}^{(x)}+\cdots+Y_{N}^{(x)}\right) \frac{K_{N}^{0}(x ; y) \Delta_{m}^{t}(x) \Delta_{m}^{t}(y)}{\prod_{1 \leq i, j \leq m}\left(1-q^{-1} x_{i} y_{j}\right)} \\
& \quad=\left(Y_{m+1}^{(y)}+\cdots+Y_{N}^{(y)}\right) \frac{K_{N}^{0}(x ; y) \Delta_{m}^{t}(x) \Delta_{m}^{t}(y)}{\prod_{1 \leq i, j \leq m}\left(1-q^{-1} x_{i} y_{j}\right)}
\end{aligned}
$$

for all $m=0, \ldots, N$, where $Y_{i}^{(x)}$ and $Y_{i}^{(y)}$ act respectively on the $x$ and $y$ variables. 
The proof of the proposition relies crucially on Lemma 17, which is formulated in terms of divided differences [21, Sec. 7.1]

$$
\partial_{i}=\frac{1}{\left(x_{i}-x_{i+1}\right)}\left(1-K_{i, i+1}\right) \text {. }
$$

The divided differences obey the braid relations [21, Sec. 7.3],

$$
\partial_{i+1} \partial_{i} \partial_{i+1}=\partial_{i} \partial_{i+1} \partial_{i}, \quad \partial_{i} \partial_{j}=\partial_{j} \partial_{i} \quad \text { if } \quad|i-j|>1,
$$

and the nilpotent condition $\partial_{i}^{2}=0$ [21, eq. 7.1.5]. As is the case for the $T_{i}$ 's, if $s_{i_{1}} \cdots s_{i_{\ell}}$ is a reduced decomposition of $w$ then $\partial_{w}$ stands for $\partial_{i_{1}} \cdots \partial_{i_{\ell}}$.

The following lemma was stated by Alain Lascoux [22]. We provide our own proof of it.

Lemma 17. Let $\partial_{\omega_{\mathrm{m}}}$ be the divided difference associated to the longest permutations of $S_{m}[21$, Sect. 7.6]. Then the following identity holds:

$$
(-t)^{-\left(\begin{array}{c}
m \\
2
\end{array}\right)} \frac{\Delta_{m}^{t}(z)}{\prod_{1 \leq i, j \leq m}\left(1-z_{i} y_{j}\right)}=\partial_{\omega_{\mathrm{m}}}^{(y)}\left(\frac{\prod_{i+j \leq m}\left(1-t z_{i} y_{j}\right)}{\prod_{i+j \leq m+1}\left(1-z_{i} y_{j}\right)}\right),
$$

where the superscript indicates that the divided differences act on the $y$ variables.

Proof. We first observe that $\prod_{1 \leq i, j \leq m}\left(1-z_{i} y_{j}\right)$, being fully symmetric in the $y_{j}$ 's, vanishes when acted on by $\partial_{\omega_{\mathrm{m}}}^{(y)}$. Therefore, multiplying both sides of the identity (127) by $\prod_{1 \leq i, j \leq m}\left(1-z_{i} y_{j}\right)$, we can then use Leibniz identity [21, eq. (7.1.10)] to commute the product with the divided differences. We then note that the product can be factorized as follows:

$$
\prod_{1 \leq i, j \leq m}\left(1-z_{i} y_{j}\right)=\prod_{i+j \leq m+1}\left(1-z_{i} y_{j}\right) \prod_{\substack{i+j>m+1 \\ i, j \leq m}}\left(1-z_{i} y_{j}\right) .
$$

Hence, the identity (127) is equivalent to

$$
\begin{aligned}
(-t)^{-\left(\begin{array}{c}
m \\
2
\end{array}\right)} \Delta_{m}^{t}(z) & =\partial_{\omega_{\mathrm{m}}}^{(y)}\left(\prod_{i+j \leq m}\left(1-t z_{i} y_{j}\right) \prod_{\substack{i+j>m+1 \\
i, j \leq m}}\left(1-z_{i} y_{j}\right)\right) \\
& =\partial_{\omega_{\mathrm{m}}}^{(y)} Q(z, y) .
\end{aligned}
$$


It is well known [21, Sect. 7.6] that

$$
\partial_{\omega_{\mathbf{m}}}^{(y)}\left(y_{1}^{a_{1}} \cdots y_{m}^{a_{m}}\right)=0
$$

unless all the $a_{k}$ 's are distinct. Observe that after expanding the products on the rhs of (129), the appearing monomials $y_{1}^{a_{1}} \cdots y_{m}^{a_{m}}$ will all be such that $a_{1}, \ldots, a_{m} \leq m-1$. Indeed, the power of $y_{j}$ equals the number of distinct factors $z_{i}$ that can appear in the coefficient of $y_{j}^{a_{j}}$ and the maximal value of this number is, with $j$ fixed,

$$
\#\{i \mid i+j \leq m\}+\#\{i \mid i+j>m+1\}=m-1
$$

The only option to have distinct $a_{k}$ 's is thus for $\left(a_{1}, \ldots, a_{m}\right)$ to be a rearrangement of $(m-1, m-2, \ldots, 1,0)$. Therefore, the rhs of (129) is a polynomial in $y_{1}, \ldots, y_{m}$ of degree 0 , that is, the result does not depend on the variables $y$. We now show that it is $t$-antisymmetric in the $z$ variables, i.e., that

$$
T_{k}^{(z)} \partial_{\omega_{\mathrm{m}}}^{(y)} Q(z, y)=-\partial_{\omega_{\mathrm{m}}}^{(y)} Q(z, y)
$$

for all $k$. We have that $\omega_{\mathbf{m}}=w s_{m-k}$ for some permutation $w \in S_{m}$. Hence, it suffices to prove that

$$
T_{k}^{(z)} \partial_{m-k}^{(y)} Q(z, y)=-\partial_{m-k}^{(y)} Q(z, y)
$$

It is easy to see that $Q(z, y)$ is symmetric in both $y_{m-k}, y_{m-k+1}$ and $z_{k}, z_{k+1}$ except for the factors $\left(1-t z_{k} y_{m-k}\right)\left(1-z_{k+1} y_{m-k+1}\right)$. A direct calculation yields

$$
\partial_{m-k}^{(y)}\left(1-t z_{k} y_{m-k}\right)\left(1-z_{k+1} y_{m-k+1}\right)=-\left(t z_{k}-z_{k+1}\right),
$$

from which (133) follows immediately since $T_{k}\left(t z_{k}-z_{k+1}\right)=-\left(t z_{k}-z_{k+1}\right)$. Finally, the rhs of (129) is a polynomial in $z$ of total degree $m(m-1) / 2$. Since the only such $t$-antisymmetric polynomial is $\Delta_{m}^{t}(z),(129)$ holds up to a constant. The coefficients of $z_{1}^{m-1} z_{2}^{m-2} \cdots z_{m-1}$ on the lhs of (129) is clearly $(-t)^{\left(\begin{array}{c}m \\ 2\end{array}\right)}$. On the rhs of $(129)$, the coefficient of $z_{1}^{m-1} z_{2}^{m-2} \cdots z_{m-1}$ is $\partial_{\omega_{\mathbf{m}}}$ acting on a certain polynomial $p(y)$ in $y$. In $p(y)$, the only monomial whose exponent is a permutation of $(m-1, m-2, \ldots, 1,0)$ is $y_{1}^{m-1} y_{2}^{m-2} \cdots y_{m-1}$. Given that its coefficient is $(-t)\left(\begin{array}{c}m \\ 2\end{array}\right)$, the result follows.

We now turn to the proof of Proposition 16. 
Proof. We will assume throughout the proof that $N>m$. The case $N=m$ can be easily obtained as a simplified version the case $N>m$. Note that in the following arguments we will never worry about constants depending on $q$ and $t$ (which are irrelevant to the symmetry). For instance, we write $U_{m}^{-(x)} \Delta_{m}^{t}(x) \propto \Delta_{m}^{t}(x)$, meaning that the two expressions only differ by a constant. (Recall that $U_{m}^{-}=\sum_{\sigma \in S_{m}}(-t)^{-\ell(\sigma)} T_{\sigma}$.)

We first prove equation (123). This amounts to show that

$$
F(x, y)=\left(\bar{Y}_{1}^{(x)}+\cdots+\bar{Y}_{m}^{(x)}\right) \frac{K_{N}^{0}(x ; y) \Delta_{m}^{t}(x) \Delta_{m}^{t}(y)}{\prod_{1 \leq i, j \leq m}\left(1-q^{-1} x_{i} y_{j}\right)}
$$

is symmetric in $x$ and $y$, that is, $F(x, y)=F(y, x)$. Since $\Delta_{m}^{t}(x) \propto$ $U_{m}^{-(x)} \Delta_{m}^{t}(x)$ and because $U_{m}^{-(x)}$ and $\Delta_{m}^{t}(y)$ commute with the $\bar{Y}_{i}^{(x)}$, s, we have

$$
F(x, y) \propto \Delta_{m}^{t}(y) U_{m}^{-(x)}\left(\bar{Y}_{1}^{(x)}+\cdots+\bar{Y}_{m}^{(x)}\right) \frac{\Delta_{m}^{t}(x) K_{N}^{0}(x ; y)}{\prod_{1 \leq i, j \leq m}\left(1-q^{-1} x_{i} y_{j}\right)} .
$$

Recall the expression for the inverse Cherednik operator in (103) and that for $\bar{T}_{j}$ in (21). Since $\bar{T}_{i}^{(x)} \Delta_{m}^{t}(x)=-t^{-1} \Delta_{m}^{t}(x)$ and $U_{m}^{-(x)} T_{i}^{(x)}=-U_{m}^{-(x)}$ whenever $i<m$ we have that, up to an irrelevant $t$-power, $U_{m}^{-(x)} \bar{Y}_{i}^{(x)} \Delta_{m}^{t}(x)$ can be replaced in (136) by $U_{m}^{-(x)} \omega^{(x)} \bar{T}_{N-1}^{(x)} \cdots \bar{T}_{m}^{(x)} \Delta_{m}^{t}(x)$. Hence

$$
F(x, y) \propto \Delta_{m}^{t}(y) U_{m}^{-(x)} \bar{\omega}^{(x)} \bar{T}_{N-1}^{(x)} \cdots \bar{T}_{m}^{(x)} \frac{\Delta_{m}^{t}(x) K_{N}^{0}(x ; y)}{\prod_{1 \leq i, j \leq m}\left(1-q^{-1} x_{i} y_{j}\right)} .
$$

Using (see [21, Sect. 7.6]) $\partial_{\omega_{\mathrm{m}}}^{(x)}=\left[\Delta_{m}(x)\right]^{-1} A_{m}$ and (31), we have

$$
U_{m}^{-(x)} \propto \Delta_{m}^{t}(x) \partial_{\omega_{\mathrm{m}}}^{(x)},
$$

which gives

$$
F(x, y) \propto \Delta_{m}^{t}(y) \Delta_{m}^{t}(x) \partial_{\omega_{\mathbf{m}}}^{(x)} \bar{\omega}^{(x)} \bar{T}_{N-1}^{(x)} \cdots \bar{T}_{m}^{(x)} \frac{\Delta_{m}^{t}(x) K_{N}^{0}(x ; y)}{\prod_{1 \leq i, j \leq m}\left(1-q^{-1} x_{i} y_{j}\right)} .
$$

Using the identity (127) with $x_{i}=q z_{i}$, we obtain

$$
F(x, y) \propto \Delta_{m}^{t}(y) \Delta_{m}^{t}(x)
$$

$$
\times \partial_{\omega_{\mathrm{m}}}^{(y)} \partial_{\omega_{\mathrm{m}}}^{(x)} \bar{\omega}^{(x)} \bar{T}_{N-1}^{(x)} \cdots \bar{T}_{m}^{(x)} \frac{\prod_{i+j \leq m}\left(1-t q^{-1} x_{i} y_{j}\right)}{\prod_{i+j \leq m+1}\left(1-q^{-1} x_{i} y_{j}\right)} K_{N}^{0}(x ; y) .
$$


To prove the symmetry of $F(x, y)$, it thus suffices to prove the symmetry of

$$
G(x, y)=\bar{\omega}^{(x)} \bar{T}_{N-1}^{(x)} \cdots \bar{T}_{m}^{(x)}\left(\frac{\prod_{i+j \leq m}\left(1-t q^{-1} x_{i} y_{j}\right)}{\prod_{i+j \leq m+1}\left(1-q^{-1} x_{i} y_{j}\right)}\right) K_{N}^{0}(x ; y)
$$

The only part of the term in parenthesis that depends on the variables $x_{m}, \ldots, x_{N}$ is $\left(1-q^{-1} x_{m} y_{1}\right)^{-1}$. We have

$$
\bar{T}_{N-1}^{(x)} \cdots \bar{T}_{m}^{(x)} \frac{1}{\left(1-q^{-1} x_{m} y_{1}\right)}=t^{-N+m} \frac{\prod_{i=m}^{N-1}\left(1-t q^{-1} x_{i} y_{1}\right)}{\prod_{i=m}^{N}\left(1-q^{-1} x_{i} y_{1}\right)}
$$

which implies that

$$
\begin{aligned}
G(x, y) \propto & \bar{\omega}^{(x)}\left(\frac{\prod_{i+j \leq m}\left(1-t q^{-1} x_{i} y_{j}\right)}{\prod_{i+j \leq m+1}\left(1-q^{-1} x_{i} y_{j}\right)}\right) \\
& \times\left(\frac{\prod_{i=m}^{N-1}\left(1-t q^{-1} x_{i} y_{1}\right)}{\prod_{i=m+1}^{N}\left(1-q^{-1} x_{i} y_{1}\right)}\right) K_{N}^{0}(x ; y) .
\end{aligned}
$$

We then straightforwardly compute

$$
\begin{gathered}
\bar{\omega}^{(x)} K_{N}^{0}(x ; y)=K_{N}^{0}(x ; y)\left[\frac{\prod_{i=1}^{N}\left(1-t q^{-1} x_{1} y_{i}\right)}{\prod_{i=1}^{N}\left(1-q^{-1} x_{1} y_{i}\right)}\right], \\
\bar{\omega}^{(x)} \prod_{i+j \leq m}\left(1-t q^{-1} x_{i} y_{j}\right)=\prod_{i+j \leq m}\left(1-t q^{-1} x_{i} y_{j}\right) \\
\times\left(\prod_{j=2}^{m-1}\left(1-t q^{-1} x_{m+1-j} y_{j}\right)\right)\left[\frac{\left(1-t q^{-1} x_{m} y_{1}\right)}{\prod_{j=1}^{m-1}\left(1-t q^{-1} x_{1} y_{j}\right)}\right] \\
\left.\bar{\omega}^{(x)} \quad \prod_{i+j \leq m+1}\left(1-q^{-1} x_{i} y_{j}\right)=\prod_{i+j \leq m+1} \frac{\left(1-q^{-1} x_{i} y_{j}\right)}{\prod_{j}}\right)\left[\frac{\left(1-q^{-1} x_{m+1} y_{1}\right)}{\prod_{j=1}^{m}\left(1-q^{-1} x_{1} y_{j}\right)}\right] \\
\quad \times\left(\prod_{j=2}^{m}\left(1-q^{-1} x_{m+2-j} y_{j}\right)\right.
\end{gathered}
$$


and

$$
\begin{aligned}
\bar{\omega}^{(x)} & \left(\frac{\prod_{i=m}^{N-1}\left(1-t q^{-1} x_{i} y_{1}\right)}{\prod_{i=m+1}^{N}\left(1-q^{-1} x_{i} y_{1}\right)}\right) \\
= & {\left[\frac{\prod_{i=m+1}^{N}\left(1-t q^{-1} x_{i} y_{1}\right)}{\prod_{i=m+2}^{N}\left(1-q^{-1} x_{i} y_{1}\right)}\right] \frac{1}{\left(1-q^{-2} x_{1} y_{1}\right)} . }
\end{aligned}
$$

All of the terms in the rhs of these expressions are symmetric in $x, y$ except those in square brackets. Multiplying the terms in square brackets, the symmetry of $G(x, y)$ then depends on the symmetry of

$$
\begin{aligned}
& \left(\frac{\prod_{i=1}^{N}\left(1-t q^{-1} x_{1} y_{i}\right)}{\prod_{i=1}^{N}\left(1-q^{-1} x_{1} y_{i}\right)}\right)\left(\frac{\left(1-t q^{-1} x_{m} y_{1}\right)}{\prod_{j=1}^{m-1}\left(1-t q^{-1} x_{1} y_{j}\right)}\right) \\
& \quad \times\left(\frac{\prod_{j=1}^{m}\left(1-q^{-1} x_{1} y_{j}\right)}{\left(1-q^{-1} x_{m+1} y_{1}\right)}\right)\left(\frac{\prod_{i=m+1}^{N}\left(1-t q^{-1} x_{i} y_{1}\right)}{\prod_{i=m+2}^{N}\left(1-q^{-1} x_{i} y_{1}\right)}\right) .
\end{aligned}
$$

But the previous expression is equal to

$$
\left(\frac{\prod_{i=m}^{N}\left(1-t q^{-1} x_{1} y_{i}\right)}{\prod_{i=m+1}^{N}\left(1-q^{-1} x_{1} y_{i}\right)}\right)\left(\frac{\prod_{i=m}^{N}\left(1-t q^{-1} x_{i} y_{1}\right)}{\prod_{i=m+1}^{N}\left(1-q^{-1} x_{i} y_{1}\right)}\right),
$$

which is obviously symmetric in $x, y$. This proves the symmetry of $G(x, y)$ and therefore equality (123) holds.

We now prove eq. (124), proceeding as in the proof of (123). This amounts to proving that

$$
H(x, y)=\left(Y_{m+1}^{(x)}+\cdots+Y_{N}^{(x)}\right) \frac{K_{N}^{0}(x ; y) \Delta_{m}^{t}(x) \Delta_{m}^{t}(y)}{\prod_{1 \leq i, j \leq m}\left(1-q^{-1} x_{i} y_{j}\right)}
$$

satisfies $H(x, y)=H(y, x)$. We have

$$
\begin{aligned}
H(x, y) \propto & \Delta_{m}^{t}(y) \\
& \times U_{m}^{-(x)} U_{m^{c}}^{+(x)}\left(Y_{m+1}^{(x)}+\cdots+Y_{N}^{(x)}\right) \frac{\Delta_{m}^{t}(x) K_{N}^{0}(x ; y)}{\prod_{1 \leq i, j \leq m}\left(1-q^{-1} x_{i} y_{j}\right)} .
\end{aligned}
$$

Since $U_{m^{c}}^{+(x)} T_{i}^{(x)}=t U_{m^{c}}^{+(x)}$ whenever $i \geq m+1$ (and similarly for $\bar{T}_{i}^{(x)}$ on 
any function symmetric in $x_{i}, x_{i+1}$ ), we have

$$
\begin{aligned}
H(x, y) \propto & \Delta_{m}^{t}(y) \\
& \times U_{m}^{-(x)} U_{m^{c}}^{+(x)} \omega^{(x)} \bar{T}_{1}^{(x)} \cdots \bar{T}_{m}^{(x)} \frac{K_{N}^{0}(x ; y)}{\prod_{1 \leq i, j \leq m}\left(1-q^{-1} x_{i} y_{j}\right)}
\end{aligned}
$$

Using (138), we have thus

$$
\begin{aligned}
H(x, y) \propto & \Delta_{m}^{t}(y) \Delta_{m}^{t}(x) \\
& \times \partial_{\omega_{\mathrm{m}}}^{(x)} U_{m^{c}}^{+(x)} \omega^{(x)} \bar{T}_{1}^{(x)} \cdots \bar{T}_{m}^{(x)} \frac{\Delta_{m}^{t}(x) K_{N}^{0}(x ; y)}{\prod_{1 \leq i, j \leq m}\left(1-q^{-1} x_{i} y_{j}\right)}
\end{aligned}
$$

Using the identity (127) with $x_{i}=q z_{i}$, we obtain

$$
\begin{aligned}
H(x, y) \propto & \Delta_{m}^{t}(y) \Delta_{m}^{t}(x) \\
& \times \partial_{\omega_{\mathbf{m}}}^{(y)} \partial_{\omega_{\mathbf{m}}}^{(x)} U_{m^{c}}^{+(x)} \omega^{(x)} \bar{T}_{1}^{(x)} \cdots \bar{T}_{m}^{(x)} \frac{\prod_{i+j \leq m}\left(1-t q^{-1} x_{i} y_{j}\right)}{\prod_{i+j \leq m+1}\left(1-q^{-1} x_{i} y_{j}\right)} K_{N}^{0}(x ; y) .
\end{aligned}
$$

To prove the symmetry of $H(x, y)$, it thus suffices to prove the symmetry of (154)

$$
L(x, y)=U_{m^{c}}^{+(x)} \omega^{(x)} \bar{T}_{1}^{(x)} \cdots \bar{T}_{m}^{(x)}\left(\frac{\prod_{i+j \leq m}\left(1-t q^{-1} x_{i} y_{j}\right)}{\prod_{i+j \leq m+1}\left(1-q^{-1} x_{i} y_{j}\right)}\right) K_{N}^{0}(x ; y) .
$$

The only part of the product that depends on the variables $x_{m}, x_{m+1}$ is $\left(1-q^{-1} x_{m} y_{1}\right)^{-1}$. We have

$$
\bar{T}_{m}^{(x)} \frac{1}{\left(1-q^{-1} x_{m} y_{1}\right)}=\frac{t^{-1}}{\left(1-q^{-1} x_{m+1} y_{1}\right)} \frac{\left(1-t q^{-1} x_{m} y_{1}\right)}{\left(1-q^{-1} x_{m} y_{1}\right)}
$$

Adding the previous expression to the product in the rhs of (154), the only factor that is not symmetric in $x_{m-1}, x_{m}$ is $\left(1-q^{-1} x_{m-1} y_{2}\right)^{-1}$. We have this time

$$
\bar{T}_{m-1}^{(x)} \frac{1}{\left(1-q^{-1} x_{m-1} y_{2}\right)}=\frac{t^{-1}}{\left(1-q^{-1} x_{m} y_{2}\right)} \frac{\left(1-t q^{-1} x_{m-1} y_{2}\right)}{\left(1-q^{-1} x_{m-1} y_{2}\right)}
$$


Continuing in this manner, we get

$$
\begin{aligned}
& \bar{T}_{1}^{(x)} \cdots \bar{T}_{m}^{(x)}\left(\frac{\prod_{i+j \leq m}\left(1-t q^{-1} x_{i} y_{j}\right)}{\prod_{i+j \leq m+1}\left(1-q^{-1} x_{i} y_{j}\right)}\right) \\
& \quad=t^{-m}\left(\frac{\prod_{i+j \leq m}\left(1-t q^{-1} x_{i} y_{j}\right)}{\prod_{i+j \leq m+1}\left(1-q^{-1} x_{i} y_{j}\right)}\right)\left(\frac{\prod_{i=1}^{m}\left(1-t q^{-1} x_{m+1-i} y_{i}\right)}{\prod_{i=1}^{m}\left(1-q^{-1} x_{m+2-i} y_{i}\right)}\right),
\end{aligned}
$$

which implies that

$L(x, y) \propto U_{m^{c}}^{+(x)} \omega^{(x)}\left(\frac{\prod_{i+j \leq m+1}\left(1-t q^{-1} x_{i} y_{j}\right)}{\prod_{i+j \leq m+2}\left(1-q^{-1} x_{i} y_{j}\right)}\right)\left(1-q^{-1} x_{1} y_{m+1}\right) K_{N}^{0}(x ; y)$.

The following actions of $\omega^{(x)}$ are straightforward:

$$
\omega^{(x)} K_{N}^{0}(x ; y)=K_{N}^{0}(x ; y)\left[\frac{\prod_{i=1}^{N}\left(1-x_{N} y_{i}\right)}{\prod_{i=1}^{N}\left(1-t x_{N} y_{i}\right)}\right]
$$

(159) $\omega^{(x)} \prod_{i+j \leq m+1}\left(1-t q^{-1} x_{i} y_{j}\right)=\prod_{i+j \leq m}\left(1-t q^{-1} x_{i} y_{j}\right)\left[\prod_{j=1}^{m}\left(1-t x_{N} y_{j}\right)\right]$,

$(160) \omega^{(x)} \prod_{i+j \leq m+2}\left(1-q^{-1} x_{i} y_{j}\right)=\prod_{i+j \leq m+1}\left(1-q^{-1} x_{i} y_{j}\right)\left[\prod_{j=1}^{m+1}\left(1-x_{N} y_{j}\right)\right]$,

and

$$
\omega^{(x)}\left(1-q^{-1} x_{1} y_{m+1}\right)=\left[\left(1-x_{N} y_{m+1}\right)\right] .
$$

The product of the terms in square brackets of these expressions gives

$$
\begin{aligned}
& {\left[\frac{\prod_{i=1}^{N}\left(1-x_{N} y_{i}\right)}{\prod_{i=1}^{N}\left(1-t x_{N} y_{i}\right)}\right]\left[\frac{\prod_{j=1}^{m}\left(1-t x_{N} y_{j}\right)}{\prod_{j=1}^{m+1}\left(1-x_{N} y_{j}\right)}\right]\left[\left(1-x_{N} y_{m+1}\right)\right]} \\
& =\frac{\prod_{i=m+1}^{N}\left(1-x_{N} y_{i}\right)}{\prod_{i=m+1}^{N}\left(1-t x_{N} y_{i}\right)} .
\end{aligned}
$$

Hence

(163)

$$
L(x, y) \propto \frac{\prod_{i+j \leq m}\left(1-t q^{-1} x_{i} y_{j}\right)}{\prod_{i+j \leq m+1}\left(1-q^{-1} x_{i} y_{j}\right)} K_{N}^{0}(x ; y) U_{m^{c}}^{+(x)}\left(\frac{\prod_{i=m+1}^{N}\left(1-x_{N} y_{i}\right)}{\prod_{i=m+1}^{N}\left(1-t x_{N} y_{i}\right)}\right) .
$$


All the terms in this expression are symmetric in $x, y$, except possibly

$$
U_{m^{c}}^{+(x)}\left(\frac{\prod_{i=m+1}^{N}\left(1-x_{N} y_{i}\right)}{\prod_{i=m+1}^{N}\left(1-t x_{N} y_{i}\right)}\right) .
$$

But the symmetry of this last expression follows from the well known symmetry of the $m=0$ case (the usual Macdonald case). Therefore $L(x, y)$ is symmetric and (124) holds.

\subsection{Duality}

We end this section by generalizing to superspace the standard duality property that relates the Macdonald symmetric functions $P_{\lambda}(q, t)$ and $P_{\lambda^{\prime}}(t, q)$ [24, Section VI.5]. Our method relies on the orthogonality and triangularity of both the Macdonald superpolynomials $P_{\Lambda}(q, t)$ and the Jack superpolynomials $P_{\Lambda}^{(\alpha)}$, respectively established in Corollary 14 above and Theorem 1 of [12]. We also exploit the duality between the Jack superpolynomials $P_{\Lambda}^{(\alpha)}$ and $P_{\Lambda^{\prime}}^{\left(\alpha^{-1}\right)}$ given in Theorem 27 of [12]. Note that in what follows, only the special case $\alpha=1$ is relevant.

The algebra $\mathscr{R}$ of symmetric functions in superspace is naturally equipped with two homomorphisms, the first of which being

$$
\omega_{q, t} p_{r}=(-1)^{r-1} \frac{1-q^{r}}{1-t^{r}} p_{r} \quad \omega_{q, t} \tilde{p}_{r}=(-1)^{r} \tilde{p}_{r} .
$$

This is an extension to superspace of the standard homomorphism defined in [24, Section VI.2]. Second, we introduce the following homomorphism that affects only the fermionic power-sums:

$$
\tilde{\omega}_{q} p_{r}=p_{r} \quad \tilde{\omega}_{q} \tilde{p}_{r}=q^{r} \tilde{p}_{r} .
$$

Combining the two homomorphisms, we get

$$
\Omega_{q, t}:=\tilde{\omega}_{q} \circ \omega_{q, t},
$$

which is such that

$$
\Omega_{q, t} p_{\Lambda}=\omega_{\Lambda} q^{\left|\Lambda^{a}\right|} \prod_{i=1}^{\ell\left(\Lambda^{s}\right)} \frac{1-q^{\Lambda_{i}^{s}}}{1-t^{\Lambda_{i}^{s}}} p_{\Lambda},
$$


where

$$
\omega_{\Lambda}=(-1)^{|\Lambda|-\ell\left(\Lambda^{s}\right)} .
$$

When $q=t=1, \Omega_{q, t}$ reduces to the homomorphism $\omega$ of [11], whose action can be summarized as follows:

$$
\omega p_{\Lambda}=\omega_{\Lambda} p_{\Lambda}
$$

Equations (168) and (170) immediately imply that, for all $f, g \in \mathscr{R}$ (and by linearity, it suffices to verify the case where $f=p_{\Lambda}$ and $g=p_{\Omega}$ ),

$$
\left\langle\left\langle\Omega_{q, t}^{-1} f \mid g\right\rangle\right\rangle_{q, t}=\langle\langle\omega f \mid g\rangle\rangle
$$

where the scalar product $\langle\langle\mid\rangle\rangle$ on the right-hand side is defined as $\langle\langle\mid\rangle\rangle_{q, t}$ in (4), but with $q=t=1$. Note that both $\Omega_{q, t}$ and $\omega$ are in fact automorphisms of $\mathscr{R}$. Their respective inverse are:

$$
\Omega_{q, t}^{-1}=\Omega_{t, q} \circ \tilde{\omega}_{(q t)^{-1}} \quad \text { and } \quad \omega^{-1}=\omega .
$$

In each homogeneous component $\mathscr{R}^{n, m}$ of $\mathscr{R}$, we also have

$$
\Omega_{q, t}^{-1}=\left(t^{n} q^{-n}\right) \Omega_{t^{-1}, q^{-1}}
$$

Now let $\mathbf{s}_{\Lambda}$ be the Schur superpolynomial associated to the Jack superpolynomial $P_{\Lambda}^{(\alpha)}[12]$, which means $\mathbf{s}_{\Lambda}:=P_{\Lambda}^{(1)}$. We stress that $\mathbf{s}_{\Lambda}$ is not equal to the Schur function $s_{\Lambda}$ defined later in the article as the $q, t \rightarrow 0$ limit of $P_{\Lambda}(q, t)$ (cf. eq. (223)). Then according to Theorem 1 of [12],

$$
\left\langle\left\langle\mathrm{s}_{\Lambda} \mid \mathrm{s}_{\Omega}\right\rangle\right\rangle=\mathrm{b}_{\Lambda}^{-1} \delta_{\Lambda, \Omega}
$$

Moreover, from [12, Theorem 27] applied to $\alpha=1$ (and recall that $\Lambda^{\prime}$ is the conjugate of $\Lambda$ ),

$$
\omega \mathrm{s}_{\Lambda}=\mathrm{s}_{\Lambda^{\prime}}^{*}=\mathrm{b}_{\Lambda}^{-1} \mathrm{~s}_{\Lambda^{\prime}}
$$

The expression for the normalization constant $\mathrm{b}_{\Lambda}^{-1}$ is known, being equal to $(-1)^{\left(\begin{array}{c}m \\ 2\end{array}\right)}\left\|P_{\Lambda}^{(1)}\right\|^{2}$, where $\left\|P_{\Lambda}^{(\alpha)}\right\|^{2}$ is given by Eq. (18) of [13]. When specialized to $\alpha=1$, this normalization factor reads

$$
\mathrm{b}_{\Lambda}=(-1)^{\left(\begin{array}{c}
m \\
2
\end{array}\right)} \prod_{s \in \Lambda} \frac{a_{\Lambda^{\circledast}}+\ell_{\Lambda^{*}}+1}{a_{\Lambda^{*}}+\ell_{\Lambda^{\circledast}}+1} .
$$


Since upon conjugation, the role of the arm-length and leg-length (defined in (8)) are exchanged, it satisfies

$$
\mathrm{b}_{\Lambda^{\prime}}=\mathrm{b}_{\Lambda}^{-1}
$$

so that we can write

$$
\mathrm{s}_{\Lambda}^{*}:=\mathrm{b}_{\Lambda} \mathrm{s}_{\Lambda} \quad \text { and } \quad\left\langle\left\langle\mathrm{s}_{\Lambda} \mid \mathbf{s}_{\Omega}^{*}\right\rangle\right\rangle=\delta_{\Lambda, \Omega}
$$

Theorem 18. Let $Q_{\Lambda}=\mathrm{b}_{\Lambda}(q, t) P_{\Lambda}(q, t)$, where $\mathrm{b}_{\Lambda}(q, t)=\left\langle\left\langle P_{\Lambda}(q, t)\right|\right.$ $\left.\left.P_{\Lambda}(q, t)\right\rangle\right\rangle_{q, t}^{-1}$. Then, the following duality holds:

$$
\Omega_{q, t} P_{\Lambda}(q, t)=\left(q t^{-1}\right)^{|\Lambda|} Q_{\Lambda^{\prime}}\left(t^{-1}, q^{-1}\right)
$$

Proof. We proceed essentially as in [24, Section VI.5]. Note that in what follows, we assume that all polynomials are homogeneous and belong to $\mathscr{R}^{n, m}$, which is finite dimensional.

Thanks to the orthogonality of the Macdonald superpolynomials established in Corollary 14 and the inversion formula (173), the stated duality property is equivalent to

$$
\left\langle\left\langle\Omega_{q, t}^{-1} P_{\Lambda^{\prime}}\left(t^{-1}, q^{-1}\right) \mid P_{\Omega}(q, t)\right\rangle\right\rangle_{q, t}=\delta_{\Lambda, \Omega}
$$

By virtue of (171) and $\omega^{-1}=\omega$, the last equation turns out to be equal to

$$
\left\langle\left\langle\omega P_{\Lambda^{\prime}}\left(t^{-1}, q^{-1}\right) \mid P_{\Omega}(q, t)\right\rangle\right\rangle=\delta_{\Lambda, \Omega} .
$$

Now, let $A(q, t)$ be the transition matrix between the $P_{\Lambda}(q, t)$ 's and the $\mathbf{s}_{\Omega}$ 's, that is

$$
P_{\Lambda}(q, t)=\sum_{\Omega} A_{\Lambda \Omega}(q, t) \mathbf{s}_{\Omega}
$$

Let also $J$ be the involutive matrix with elements $J_{\Lambda \Omega}=\delta_{\Lambda^{\prime}, \Omega}$. Then (181) is equivalent to the following matrix equation:

$$
J A\left(t^{-1}, q^{-1}\right) J A(q, t)^{\prime}=I,
$$

where we stress that $A(q, t)^{\prime}$ is the transpose of the matrix $A(q, t)$. This is the equation we will prove. Before attacking this problem directly, we need to derive a number of auxiliary results, to which we now turn. 
Let $p, \mathbf{s}$, and $\mathbf{s}^{*}$ be the column vectors with the $\Lambda$-element equal to $p_{\Lambda}$, $\mathbf{s}_{\Lambda}$, and $\mathbf{s}_{\Lambda}^{*}$ respectively. Let $\mathrm{b}, \zeta$, and $\zeta(q, t)$ be diagonal matrices whose nonzero elements are respectively given by $\mathrm{b}_{\Lambda}$ (defined in (176)), $\zeta_{\Lambda}=\left\langle\left\langle p_{\Lambda} \mid p_{\Lambda}\right\rangle\right\rangle$ and $\zeta_{\Lambda}(q, t)=\left\langle\left\langle p_{\Lambda} \mid p_{\Lambda}\right\rangle_{q, t}\right.$, so that

$$
\zeta_{\Lambda}=(-1)^{\left(\begin{array}{c}
m \\
2
\end{array}\right)} z_{\Lambda^{s}} \quad \text { and } \quad \zeta_{\Lambda}(q, t)=(-1)^{\left(\begin{array}{c}
m \\
2
\end{array}\right)} z_{\Lambda^{s}} q^{\left|\Lambda^{\alpha}\right|} \prod_{i=1}^{\ell\left(\Lambda^{s}\right)} \frac{1-q^{\Lambda_{i}^{s}}}{1-t^{\Lambda_{i}^{s}}} .
$$

We also define $X$ as the matrix with entries $X_{\Lambda \Omega}=\left\langle\left\langle p_{\Lambda} \mid \mathbf{s}_{\Omega}^{*}\right\rangle\right\rangle$. Then by making use of the orthogonality with respect to $\langle\langle\mid\rangle\rangle$ of both the $s_{\Lambda}$ 's and the $p_{\Lambda}$ 's, one readily obtains

$$
p=X \mathbf{s}, \quad \mathbf{s}^{*}=X^{\prime} \zeta^{-1} p .
$$

Together with $\mathbf{s}^{*}=\mathrm{bs}$, these imply

$$
X^{\prime}=\mathrm{b} X^{-1} \zeta
$$

Moreover, let $U(q, t)$ and be the matrix with elements

$$
U_{\Lambda \Omega}=\left\langle\left\langle\mathbf{s}_{\Lambda} \mid \mathbf{s}_{\Omega}^{*}\right\rangle\right\rangle_{q, t} .
$$

The use of the previous two equations then leads to

$$
X U(q, t) X^{-1}=\zeta^{-1} \zeta(q, t),
$$

whose inverse version reads

$$
X U(q, t)^{-1} X^{-1}=\zeta(q, t)^{-1} \zeta .
$$

From the explicit expressions of $\zeta(q, t)$ and $\zeta$ in (184), we see that

$$
\zeta(q, t)^{-1} \zeta=\left(t^{n} q^{-n}\right) \zeta^{-1} \zeta\left(t^{-1}, q^{-1}\right) .
$$

Because $X$ is independent of $q$ and $t$, the relation (189) implies that

$$
U(q, t)^{-1}=\left(t^{n} q^{-n}\right) U\left(t^{-1}, q^{-1}\right) .
$$

Furthermore, we have, using (175), the adjoint character of $\omega$ and $\omega^{2}=1$, we have

$$
(J U(q, t) J)_{\Lambda \Omega}=\left\langle\left\langle\mathbf{s}_{\Lambda^{\prime}} \mid \mathbf{s}_{\Omega^{\prime}}^{*}\right\rangle\right\rangle_{q, t}=\left\langle\left\langle\omega \mathbf{s}_{\Lambda}^{*} \mid \omega \mathbf{s}_{\Omega}\right\rangle\right\rangle_{q, t}=\left\langle\left\langle\mathbf{s}_{\Lambda}^{*} \mid \mathbf{s}_{\Omega}\right\rangle\right\rangle_{q, t},
$$


so that (translating the first and third equality above)

$$
J U(q, t) J=U(q, t)^{\prime}=\mathrm{b} U(q, t) \mathrm{b}^{-1} .
$$

One last identity concerning $U(q, t)$ is needed:

$$
A(q, t) \mathrm{b}^{-1} U(q, t)^{\prime} A(q, t)^{\prime}=\mathrm{b}(q, t)^{-1},
$$

where $\mathrm{b}(q, t)^{-1}$ is the diagonal matrix with entries $\left\langle\left\langle P_{\Lambda}(q, t) \mid P_{\Lambda}(q, t)\right\rangle\right\rangle_{q, t}$. The relation (194) follows directly from the orthogonality of the Macdonald superpolynomials and the definition of $A(q, t)$.

We are now in position to prove (183). From the triangular expansions of $P_{\Lambda}(q, t)$ and $\mathrm{s}_{\Lambda}$ (which are both of the form $m_{\Lambda}+$ lower terms), we know that the matrix $A(q, t)$ is strictly upper unitriangular. We recall (see for instance [24, I.(6.2)]) that a matrix $M$ is strictly upper (uni)triangular if and only if $J M J$ is strictly lower (uni)triangular. Hence, the matrix

$$
B=J A\left(t^{-1}, q^{-1}\right) J A(q, t)^{\prime}
$$

is strictly lower unitriangular. Therefore, in order to prove that $B$ is the identity (which will prove (183)), it only remains to prove that $B$ is also upper triangular. The use of the second equality of (194) and (193), under the form $U(q, t) \mathrm{b}^{-1} J=\mathrm{b}^{-1} J U(q, t)$, allows us to write

$$
\mathrm{b}(q, t)^{-1} B^{-1}=A(q, t) \mathrm{b}^{-1} J U(q, t) A\left(t^{-1}, q^{-1}\right)^{-1} J .
$$

Now, by isolating $U$ from (194) and inverting the result by using (191), we get

$$
\mathrm{b}(q, t)^{-1} B^{-1}=\left(t^{-n} q^{n}\right) A(q, t) \mathrm{b}^{-1} J^{-1} A\left(t^{-1}, q^{-1}\right)^{\prime} \mathrm{b}\left(t^{-1}, q^{-1}\right) J .
$$

However, by exploiting (177) and the definition of $J$, one readily shows that $\mathrm{b}^{-1} \mathrm{Jb}^{-1}=J$. Thus,

$$
\mathrm{b}(q, t)^{-1} B^{-1}=\left(t^{-n} q^{n}\right) A(q, t) J A\left(t^{-1}, q^{-1}\right)^{\prime} b\left(t^{-1}, q^{-1}\right) J,
$$

The comparison with (195) yields

$$
\mathrm{b}(q, t)^{-1} B^{-1}=\left(t^{-n} q^{n}\right) B^{\prime} J \mathrm{~b}\left(t^{-1}, q^{-1}\right) J .
$$

Since both $\mathrm{b}\left(t^{-1}, q^{-1}\right)$ and $J \mathrm{~b}\left(t^{-1}, q^{-1}\right) J$ are diagonal matrices, and since $B$ is strictly lower unitriangular, the last equation implies that $B^{-1}$ is also upper triangular. Consequently, $B$ is the identity matrix, which completes the proof of (183). 


\section{Another scalar product}

The value of the norm $\left\langle\left\langle P_{\Lambda} \mid P_{\Lambda}\right\rangle_{q, t}\right.$ of the Macdonald polynomials in superspace was conjectured in [7]. This conjecture is reproduced below. We will now define another scalar product with respect to which the Macdonald polynomials in superspace are also orthogonal. This other scalar product is not bilinear anymore (it is sesquilinear). Nevertheless, we will show that, remarkably, the norm of the Macdonald polynomials in superspace with respect to that other scalar product is (up to a power of $q$ ) identical to the conjectural expression for $\left\langle\left\langle P_{\Lambda} \mid P_{\Lambda}\right\rangle_{q, t}\right.$.

\subsection{The conjectured norm of the Macdonald superpolynomials}

We first present the conjectural expression for $\left\langle\left\langle P_{\Lambda} \mid P_{\Lambda}\right\rangle\right\rangle_{q, t}$ given in [7]. It involves the quantities (recall that arm- and leg-lengths were defined in (8))

$(200) h_{\Lambda}^{\uparrow}=\prod_{s \in \mathcal{B}(\Lambda)}\left(1-q^{a_{\Lambda^{*}}(s)+1} t^{l_{\Lambda \circledast}(s)}\right), \quad h_{\Lambda}^{\downarrow}=\prod_{s \in \mathcal{B}(\Lambda)}\left(1-q^{a_{\Lambda \circledast}(s)} t^{l_{\Lambda^{*}}(s)+1}\right)$,

where $\mathcal{B}(\Lambda)$ denotes the set of squares in the diagram of $\Lambda$ that do not appear at the same time in a row containing a circle and in a column containing a circle (this excludes for instance the squares $(1,1),(1,2)$ and $(3,1)$ of $\Lambda$ whose diagram is found in (9)).

Conjecture 19. The norm of $P_{\Lambda}$ defined in Theorem 1 is

$$
\left\langle\left\langle P_{\Lambda} \mid P_{\Lambda}\right\rangle\right\rangle_{q, t}=(-1)^{\left(\begin{array}{c}
m \\
2
\end{array}\right)} q^{\left|\Lambda^{a}\right|} \frac{h_{\Lambda}^{\uparrow}}{h_{\Lambda}^{\downarrow}} .
$$

\subsection{The constant-term scalar product of the non-symmetric Macdonald polynomials}

Let C.T. $(f)$ denote the constant term of the Laurent series of $f$ in the variables $x_{1}, \ldots, x_{N}$. Define the following scalar product on $\mathbb{Q}(q, t)\left[x_{1}, \ldots, x_{N}\right]$ :

$$
\langle f, g\rangle_{N, q, t}:=\text { C.T. }\left\{f(x ; q, t) g\left(x^{-1} ; q^{-1}, t^{-1}\right) W(x ; q, t)\right\},
$$

where

$$
W(x ; q, t)=\prod_{1 \leq i<j \leq N} \frac{\left(x_{i} / x_{j} ; q\right)_{\infty}\left(q x_{j} / x_{i} ; q\right)_{\infty}}{\left(t x_{i} / x_{j} ; q\right)_{\infty}\left(q t x_{j} / x_{i} ; q\right)_{\infty}} .
$$


Note that this scalar product is sesquilinear, that is, for $c=c(q, t) \in \mathbb{Q}(q, t)$, we have

$$
\langle c f, g\rangle_{N, q, t}=c\langle f, g\rangle_{N, q, t} \quad \text { and } \quad\langle f, c g\rangle_{N, q, t}=\bar{c}\langle f, g\rangle_{N, q, t},
$$

where $\bar{c}=c(1 / q, 1 / t)$.

Proposition 20. [24, 25] The non-symmetric Macdonald polynomials $E_{\eta}(x ; q, t)$ form an orthogonal set with respect to $\langle\cdot, \cdot\rangle_{N, q, t}$. $[8,3])$

The norm is explicitly given by the expression (cf. [27, Prop. 3.4] and

$$
\frac{\left\langle E_{\eta}, E_{\eta}\right\rangle_{N, q, t}}{\langle 1,1\rangle_{N, q, t}}=\frac{d_{\eta}^{\prime} e_{\eta}}{d_{\eta} e_{\eta}^{\prime}},
$$

where for a composition $\gamma$,

$$
\begin{array}{ll}
d_{\gamma}=\prod_{s \in \gamma}\left[1-q^{a(s)+1} t^{l(s)+1}\right], & d_{\gamma}^{\prime}=\prod_{s \in \gamma}\left[1-q^{a(s)+1} t^{l(s)}\right], \\
e_{\gamma}=\prod_{s \in \gamma}\left[1-q^{a^{\prime}(s)+1} t^{N-l^{\prime}(s)}\right], & e_{\gamma}^{\prime}=\prod_{s \in \gamma}\left[1-q^{a^{\prime}(s)+1} t^{N-1-l^{\prime}(s)}\right], \\
b_{\gamma}=\prod_{s \in \gamma}\left[1-q^{a^{\prime}(s)} t^{N-l^{\prime}(s)}\right], & h_{\gamma}=\prod_{s \in \gamma}\left[1-q^{a(s)} t^{l(s)+1}\right],
\end{array}
$$

(we added two expressions to be needed shortly, $b_{\gamma}$ and $h_{\gamma}$ ). The arm- and leg-(co)lengths in these expressions are given, for $s=(i, j)$, by

$$
\begin{aligned}
& a(s)=\gamma_{i}-j, \\
& l(s)=\#\left\{k=1, \ldots, i-1 \mid j \leq \gamma_{k}+1 \leq \gamma_{i}\right\} \\
& +\#\left\{k=i+1, \ldots, N \mid j \leq \gamma_{k} \leq \gamma_{i}\right\}, \\
& a^{\prime}(s)=j-1 \text {, } \\
& l^{\prime}(s)=\#\left\{k=1, \ldots, i-1 \mid \gamma_{k} \geq \gamma_{i}\right\} \\
& +\#\left\{k=i+1, \ldots, N \mid \gamma_{k}>\gamma_{i}\right\} \text {. }
\end{aligned}
$$

\subsection{Another orthogonality relation for the Macdonald superpolynomials}

Let $f \in \mathscr{R}_{N}$ be a symmetric superpolynomial of fermionic degree $m$. We define

$$
f^{\bullet}=t^{-\left(\begin{array}{c}
m \\
2
\end{array}\right)} \frac{\Delta_{m}^{t}(x)}{\Delta_{m}(x)}\left[\theta_{1} \ldots \theta_{m}\right] f
$$


where we recall that, as in Section $4,\left[\theta_{1} \cdots \theta_{m}\right] f$ stands for the coefficient of $\theta_{1} \cdots \theta_{m}$ in $f$. Note that $f^{\bullet} \in \mathbb{Q}(q, t)\left[x_{1}, \ldots, x_{N}\right]$ since $\left[\theta_{1} \cdots \theta_{m}\right] f$ is antisymmetric in the variables $x_{1}, \ldots, x_{m}$ and thus divisible by $\Delta_{m}(x)$. If $f$ does not have a specific fermionic degree, it can be decomposed as $f=f_{0}+f_{1}+\cdots+f_{r}$, where $f_{m}$ is the part of $f$ of fermionic degree $m$. We then let

$$
f^{\bullet}=f_{0}^{\bullet}+f_{1}^{\bullet}+\cdots+f_{r}^{\bullet}
$$

Definition 21. Let $f$ and $g$ be superpolynomials in $\mathscr{R}_{N}$. We define the following sesquilinear scalar product on $\mathscr{R}$ :

$$
\langle f, g\rangle_{N, q, t}^{\mathscr{R}}=\sum_{m} \frac{1}{[m]_{t} !}\left\langle f_{m}^{\bullet}, g_{m}^{\bullet}\right\rangle_{N, q, t}
$$

The following proposition states that the Macdonald superpolynomials are also orthogonal with respect to this new scalar product. Moreover, it relates the norm of $P_{\Lambda}$ in the two scalar products.

Proposition 22. We have

$$
\left\langle P_{\Lambda}, P_{\Omega}\right\rangle_{N, q, t}^{\mathscr{R}}=0 \quad \text { if } \Lambda \neq \Omega .
$$

Furthermore,

$$
\lim _{N \rightarrow \infty} \frac{\left\langle P_{\Lambda}, P_{\Lambda}\right\rangle_{N, q, t}^{\mathscr{R}}}{\langle 1,1\rangle_{N, q, t}}=(-1)^{\left(\begin{array}{c}
m \\
2
\end{array}\right)} \frac{h_{\Lambda}^{\uparrow}}{h_{\Lambda}^{\downarrow}} \stackrel{?}{=} q^{-\left|\Lambda^{a}\right|}\left\langle\left\langle P_{\Lambda} \mid P_{\Lambda}\right\rangle\right\rangle_{q, t},
$$

where $m$ is the fermionic degree of $\Lambda$, and where $\stackrel{?}{=}$ means that the equality is only conjectural.

Proof. By definition $\left\langle P_{\Lambda}, P_{\Omega}\right\rangle_{N, q, t}^{\mathscr{R}}=0$ if $\Lambda$ and $\Omega$ have different fermionic degrees. We can thus suppose that $\Lambda$ and $\Omega$ have fermionic degree $m$. The proof now follows the argument of [3] up to eq. (220). Using expression (217), we get

$$
\begin{aligned}
& \left\langle P_{\Lambda}, P_{\Omega}\right\rangle_{N, q, t}^{\mathscr{R}} \\
& \quad=\frac{c_{\Lambda}(t) c_{\Omega}\left(t^{-1}\right)}{[m]_{t} !} \sum_{\sigma \in S_{m}} \sum_{\sigma^{\prime} \in S_{m^{c}}}\left\langle U_{m}^{-} U_{m^{c}}^{+} E_{\Lambda^{R}},(-t)^{-\ell(\sigma)} T_{\sigma} T_{\sigma^{\prime}} E_{\Omega^{R}}\right\rangle_{N, q, t} \\
& \quad=\frac{c_{\Lambda}(t) c_{\Omega}\left(t^{-1}\right)}{[m]_{t} !} \sum_{\sigma \in S_{m}} \sum_{\sigma^{\prime} \in S_{m^{c}}}\left\langle(-t)^{\ell(\sigma)} T_{\sigma^{\prime-1}}^{-1} T_{\sigma^{-1}}^{-1} U_{m}^{-} U_{m^{c}}^{+} E_{\Lambda^{R}}, E_{\Omega^{R}}\right\rangle_{N, q, t},
\end{aligned}
$$


where we used the fact that $T_{\sigma^{-1}}^{-1}$ is the adjoint of $T_{\sigma}$ with respect to $\langle\cdot, \cdot\rangle_{N, q, t}$. Note that $T_{\sigma}$ and $T_{\sigma^{\prime}}$ commute because they act on disjoint sets of variables. We then use

$$
T_{i}^{ \pm} U^{+}=t^{ \pm} U^{+} \quad \text { and } \quad T_{i}^{ \pm} U^{-}=-U^{-}
$$

in order to write

$$
\left\langle P_{\Lambda}, P_{\Omega}\right\rangle_{N, q, t}^{\mathscr{R}}=[N-m]_{t^{-1}} ! c_{\Omega}\left(t^{-1}\right)\left\langle S_{\left(\Lambda^{a}, \Lambda^{s}\right)}, E_{\Omega^{R}}\right\rangle_{N, q, t},
$$

where we used $\sum_{\sigma \in S_{r}} t^{\ell(\sigma)}=[r]_{t}$ !, and where

$$
S_{\left(\Lambda^{a}, \Lambda^{s}\right)}=c_{\Lambda} U_{m}^{-} U_{m^{c}}^{+} E_{\left(\left(\Lambda^{a}\right)^{R},\left(\Lambda^{s}\right)^{R}\right)},
$$

is a Macdonald polynomial with mixed symmetry (also considered in [3] but only in the case where $\left.\lambda=\delta^{(m)}=(m-1, \ldots, 0)\right)$. The analogue of [1, Corr. $1]$, which is obtained as shown there using the generalization of lemma 2.5 of [27], reads

$$
S_{(\lambda, \mu)}=\sum_{\sigma, \sigma^{\prime}}(-t)^{-\ell\left(\sigma^{\prime}\right)} \frac{d_{\left(\lambda^{R}, \mu\right)}^{\prime} d_{\left(\sigma^{\prime}\left(\lambda^{R}\right), \sigma\left(\mu^{R}\right)\right)}}{d_{\left(\lambda^{R}, \sigma\left(\mu^{R}\right)\right)}^{\prime} d_{\left(\lambda, \sigma\left(\mu^{R}\right)\right)}} E_{\left(\sigma^{\prime}\left(\lambda^{R}\right), \sigma\left(\mu^{R}\right)\right)} .
$$

By the orthogonality of the non-symmetric Macdonald polynomials, the rhs of (215) is non-vanishing only when $\Lambda=\Omega$, and $\sigma$ and $\sigma^{\prime}$ are equal to the identity in (217). Hence

$$
\begin{aligned}
\left\langle P_{\Lambda},\left.P_{\Omega}\right|_{N, q, t} ^{\mathscr{R}}=\right. & \delta_{\Lambda \Omega}(-1)^{\left(\begin{array}{c}
m \\
2
\end{array}\right)} \frac{[N-m]_{t^{-1}} ! t^{\operatorname{inv}\left(\Lambda^{s}\right)}}{f_{\Lambda^{s}}\left(t^{-1}\right)} \\
& \times \frac{d_{\left(\left(\Lambda^{a}\right)^{R}, \Lambda^{s}\right)}^{\prime} d_{\left(\left(\Lambda^{a}\right)^{R},\left(\Lambda^{s}\right)^{R}\right)}}{d_{\left(\left(\Lambda^{a}\right)^{R},\left(\Lambda^{s}\right)^{R}\right)}^{\prime} d_{\left.\left(\Lambda^{a}, \Lambda^{s}\right)^{R}\right)}}\left\langle E_{\Lambda^{R}}, E_{\Lambda^{R}}\right\rangle_{N, q, t},
\end{aligned}
$$

which implies that $\left\langle P_{\Lambda}, P_{\Omega}\right\rangle_{N, q, t}^{\mathscr{R}}=0$ if $\Lambda \neq \Omega$.

Furthermore, according to the identities given in [27, eqs (5.16) and $(5.15)]$,

$$
\frac{[N-m]_{t^{-1}} !}{f_{\Lambda^{s}}\left(t^{-1}\right)} t^{\operatorname{inv}\left(\Lambda^{s}\right)}=\frac{[N-m]_{t} !}{f_{\Lambda^{s}}(t)}=\frac{b_{\Lambda^{s}} d_{\left(\Lambda^{s}\right)^{R}}}{h_{\Lambda^{s}} e_{\left(\Lambda^{s}\right)^{R}}}
$$

so that, using the expression (205) for the norm of $E_{\Lambda^{R}}$, we obtain

$$
\frac{\left\langle P_{\Lambda}, P_{\Lambda}\right\rangle_{N, q, t}^{\mathscr{R}}}{\langle 1,1\rangle_{N, q, t}}=(-1)^{\left(\begin{array}{c}
m \\
2
\end{array}\right)} \frac{b_{\Lambda^{s}} d_{\left(\Lambda^{s}\right)^{R}}}{h_{\Lambda^{s}} e_{\left(\Lambda^{s}\right)^{R}}} \frac{d_{\left(\left(\Lambda^{a}\right)^{R}, \Lambda^{s}\right)}^{\prime}}{d_{\left(\Lambda^{a},\left(\Lambda^{s}\right)^{R}\right)}} \frac{e_{\left(\left(\Lambda^{a}\right)^{R},\left(\Lambda^{s}\right)^{R}\right)}}{e_{\left(\left(\Lambda^{a}\right)^{R},\left(\Lambda^{s}\right)^{R}\right)}^{\prime}} .
$$


In order to relate this expression to the conjectural expression for $\left\langle\left\langle P_{\Lambda} \mid P_{\Lambda}\right\rangle\right\rangle_{q, t}$, we need to consider the limit $N \rightarrow \infty$ of $(220)$ and recall that $|t|<1$, so that all the factors $e, e^{\prime}$ and $b$ reduce to 1 . The proposition will follow after establishing that

(221) $\lim _{N \rightarrow \infty} \frac{\left\langle P_{\Lambda}, P_{\Lambda}\right\rangle_{N, q, t}^{\mathscr{R}}}{\langle 1,1\rangle_{N, q, t}}=(-1)^{\left(\begin{array}{c}m \\ 2\end{array}\right)} \lim _{N \rightarrow \infty} \frac{d_{\left(\left(\Lambda^{a}\right)^{R}, \Lambda^{s}\right)}^{\prime} d_{\left(\Lambda^{s}\right)^{R}}}{d_{\left(\Lambda^{a},\left(\Lambda^{s}\right)^{R}\right)} h_{\Lambda^{s}}}=(-1)^{\left(\begin{array}{c}m \\ 2\end{array}\right)} \frac{h_{\Lambda}^{\uparrow}}{h_{\Lambda}^{\downarrow}}$.

The last equality is quite long to prove. The details are thus relegated to the appendix.

\section{Macdonald superpolynomials and generalized $(q, t)$-Kostka coefficients}

In this section, we review the super-extension of the Macdonald positivity conjecture, exhibit symmetries of the generalized $(q, t)$-Kostka coefficients and present two new conjectures related to these coefficients.

\subsection{Generalized $(q, t)$-Kostka coefficients}

The generalized $(q, t)$-Kostka coefficients are defined from the integral form of the Macdonald superpolynomials:

$$
J_{\Lambda}=h_{\Lambda}^{\downarrow} P_{\Lambda}
$$

(where $h_{\Lambda}^{\downarrow}$ is defined in (200)). It was conjectured in [7] that the coefficients in the monomial expansion of $J_{\Lambda}$ are polynomials in $q$ and $t$ with integer coefficients.

We next introduce the Schur superpolynomials [7]

$$
s_{\Lambda}(x, \theta)=P_{\Lambda}(x, \theta ; 0,0),
$$

and their deformation

$$
S_{\Lambda}(x, \theta ; t)=\varphi\left(s_{\Lambda}(x, \theta)\right),
$$

where $\varphi$ stands for the endomorphism of $\mathbb{Q}(q, t)\left[p_{1}, p_{2}, p_{3}, \ldots ; \tilde{p}_{0}, \tilde{p}_{1}, \tilde{p}_{2}, \ldots\right]$ defined by its action on the power-sums as

$$
\varphi\left(p_{n}\right)=\left(1-t^{n}\right) p_{n} \quad \text { and } \quad \varphi\left(\tilde{p}_{n}\right)=\tilde{p}_{n} .
$$


Remark 23. As was commented in [7], the existence of the limiting case $s_{\Lambda}(x, \theta)=P_{\Lambda}(x, \theta ; 0,0)$ does not follow from the existence of a solution from Theorem 1 since the scalar product is degenerate when $q=t=0$. A better approach is to consider the limit $q=t \rightarrow 0$ in Definition 33. This is presented in Appendix A. Specifically, we prove the existence of the Schur superpolynomials by using the fact that in the limit $q=t \rightarrow 0$, a nonsymmetric Macdonald polynomial tends to a Key polynomial (or Demazure character) $[16,23]$.

We now recall the following striking version of the Macdonald positivity conjecture, formulated in [7].

Conjecture 24. The coefficients $K_{\Omega \Lambda}(q, t)$ in the expansion of the integral form of the Macdonald superpolynomials

$$
J_{\Lambda}(x, \theta ; q, t)=\sum_{\Omega} K_{\Omega \Lambda}(q, t) S_{\Omega}(x, \theta ; t)
$$

are polynomials in $q$ and $t$ with nonnegative integer coefficients.

The following symmetries have been observed:

$$
K_{\Omega \Lambda}(q, t)=K_{\Omega^{\prime} \Lambda^{\prime}}(t, q),
$$

(cf. [24, eq. VI (8.15)]) and

$$
K_{\Omega \Lambda}(q, t)=q^{\bar{n}\left(\Lambda^{\prime}\right)} t^{\bar{n}(\Lambda)} K_{\Omega^{\prime} \Lambda}\left(q^{-1}, t^{-1}\right) .
$$

In the previous equation we used

$$
\bar{n}(\Lambda)=n(\mathcal{S} \Lambda)-d(\Lambda) \quad \text { with } \quad n(\lambda)=\sum_{i}(i-1) \lambda_{i}
$$

where $\mathcal{S} \Lambda$ is the skew diagram $\mathcal{S} \Lambda=\Lambda^{\circledast} / \delta^{(m+1)}$, with $\delta^{(m)}$ the staircase partition $(m-1, m-2, \ldots, 1,0)$, and where $d(\Lambda)$ is defined as follows: fill each square $s \in \mathcal{B} \Lambda$ (defined immediately after (200)) by the number corresponding to the number of squares above $s$ which are both in a fermionic row and a fermionic column (i.e., both ending with a circle); $d(\Lambda)$ is then the sum of these entries. For example,

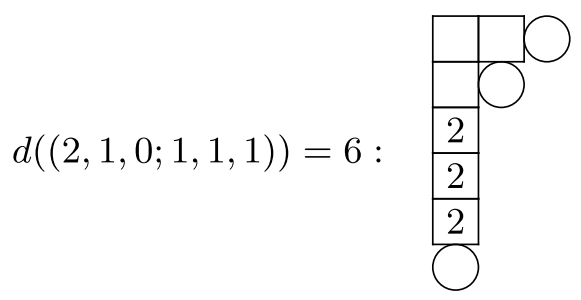




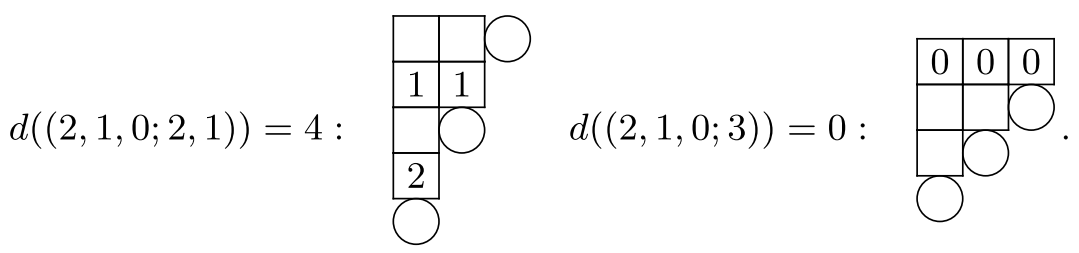

(In a sense, $d(\Lambda)$ is dual to the quantity $\zeta_{\Lambda}$ introduced in [7] to describe the specialization of $P_{\Lambda}$.) The quantity $d(\Lambda)$ vanishes for $m \leq 1$, so that that expression $[24$, eq. VI (8.14)] is recovered when $m=0$. Examples of $(q, t)$-Kostka coefficients are given in Tables 1 to 7 . We stress that in these tables, the prime stands for the matrix transpose, so that the matrix element found in row $\Lambda$ and column $\Omega$ gives the coefficient $K_{\Lambda, \Omega}^{\prime}=K_{\Omega, \Lambda}$ of $S_{\Omega}$ in the modified Macdonald superpolynomial $J_{\Lambda}$. Note also that in the tables

Table 1: $K(q, t)^{\prime}$ for degree $(1 \mid 1)$

\begin{tabular}{c|c|c|} 
& $(1 ;)$ & $(0 ; 1)$ \\
\hline$(1 ;)$ & 1 & $q$ \\
\hline$(0 ; 1)$ & $t$ & 1 \\
\hline
\end{tabular}

Table 2: $K(q, t)^{\prime}$ for degree $(2 \mid 1)$

\begin{tabular}{c|c|c|c|c|} 
& $(2 ;)$ & $(0 ; 2)$ & $(1 ; 1)$ & $(0 ; 1,1)$ \\
\hline$(2 ;)$ & 1 & $q^{2}$ & $q$ & $q^{3}$ \\
\hline$(0 ; 2)$ & $t$ & 1 & $q t$ & $q$ \\
\hline$(1 ; 1)$ & $t$ & $q t$ & 1 & $q$ \\
\hline$(0 ; 1,1)$ & $t^{3}$ & $t$ & $t^{2}$ & 1 \\
\hline
\end{tabular}

Table 3: $K(q, t)^{\prime}$ for degree $(2 \mid 2)$

\begin{tabular}{c|c|c|} 
& $(2,0 ;)$ & $(1,0 ; 1)$ \\
\hline$(2,0 ;)$ & 1 & $q$ \\
\hline$(1,0 ; 1)$ & $t$ & 1 \\
\hline
\end{tabular}

Table 4: $K(q, t)^{\prime}$ for degree $(3 \mid 1)$

\begin{tabular}{c|c|c|c|c|c|c|c|} 
& $(3 ;)$ & $(0 ; 3)$ & $(2 ; 1)$ & $(1 ; 2)$ & $(0 ; 2,1)$ & $(1 ; 1,1)$ & $(0 ; 1,1,1)$ \\
\hline$(3 ;)$ & 1 & $q^{3}$ & $q+q^{2}$ & $q^{2}+q^{4}$ & $q^{4}+q^{5}$ & $q^{3}$ & $q^{6}$ \\
\hline$(0 ; 3)$ & $t$ & 1 & $q t+q^{2} t$ & $q+q^{2} t$ & $q+q^{2}$ & $q^{3} t$ & $q^{3}$ \\
\hline$(2 ; 1)$ & $t$ & $q^{2} t$ & $1+q t$ & $q+q^{2} t$ & $q^{2}+q^{3} t$ & $q$ & $q^{3}$ \\
\hline$(1 ; 2)$ & $t^{2}$ & $q t$ & $t+q t^{2}$ & $1+q^{2} t^{2}$ & $q+q^{2} t$ & $q t$ & $q^{2}$ \\
\hline$(0 ; 2,1)$ & $t^{3}$ & $t$ & $t^{2}+q t^{3}$ & $t+q t^{2}$ & $1+q t$ & $q t^{2}$ & $q$ \\
\hline$(1 ; 1,1)$ & $t^{3}$ & $q t^{3}$ & $t+t^{2}$ & $t+q t^{2}$ & $q t+q t^{2}$ & 1 & $q$ \\
\hline$(0 ; 1,1,1)$ & $t^{6}$ & $t^{3}$ & $t^{4}+t^{5}$ & $t^{2}+t^{4}$ & $t+t^{2}$ & $t^{3}$ & 1 \\
\hline
\end{tabular}


Table 5: $K(q, t)^{\prime}$ for degree $(3 \mid 2)$

\begin{tabular}{c|c|c|c|c|c|} 
& $(3,0 ;)$ & $(2,1 ;)$ & $(2,0 ; 1)$ & $(1,0 ; 2)$ & $(1,0 ; 1,1)$ \\
\hline$(3,0 ;)$ & 1 & $q$ & $q+q^{2}$ & $q^{2}$ & $q^{3}$ \\
\hline$(2,1 ;)$ & $q t$ & 1 & $q+q^{2} t$ & $q^{3} t$ & $q^{2}$ \\
\hline$(2,0 ; 1)$ & $t$ & $t$ & $1+q t$ & $q$ & $q$ \\
\hline$(1,0 ; 2)$ & $t^{2}$ & $q t^{3}$ & $t+q t^{2}$ & 1 & $q t$ \\
\hline$(1,0 ; 1,1)$ & $t^{3}$ & $t^{2}$ & $t+t^{2}$ & $t$ & 1 \\
\hline
\end{tabular}

Table 6: $K(q, t)^{\prime}$ for degree $(4 \mid 1)$

\begin{tabular}{l|c|c|c|c|c|c|} 
& $(4 ;)$ & $(0 ; 4)$ & $(3 ; 1)$ & $(1 ; 3)$ & $(0 ; 3,1)$ & $(2 ; 2)$ \\
\hline$(4 ;)$ & 1 & $q^{4}$ & $q+q^{2}+q^{3}$ & $q^{3}+q^{5}+q^{6}$ & $q^{5}+q^{6}+q^{7}$ & $q^{2}+q^{4}$ \\
\hline$(0 ; 4)$ & $t$ & 1 & $q t+q^{2} t+q^{3} t$ & $q+q^{2}+q^{3} t$ & $q+q^{2}+q^{3}$ & $q^{2} t+q^{4} t$ \\
\hline$(3 ; 1)$ & $t$ & $q^{3} t$ & $1+q t+q^{2} t$ & $q^{2}+q^{3} t+q^{4} t$ & $q^{3}+q^{4} t+q^{5} t$ & $q+q^{2} t$ \\
\hline$(1 ; 3)$ & $t^{2}$ & $q t$ & $t+q t^{2}+q^{2} t^{2}$ & $1+q^{2} t+q^{3} t^{2}$ & $q+q^{2} t+q^{3} t$ & $q t+q^{2} t^{2}$ \\
\hline$(0 ; 3,1)$ & $t^{3}$ & $t$ & $t^{2}+q t^{3}+q^{2} t^{3}$ & $t+q t+q^{2} t^{2}$ & $1+q t+q^{2} t$ & $q t^{2}+q^{2} t^{3}$ \\
\hline$(2 ; 2)$ & $t^{2}$ & $q^{2} t^{2}$ & $t+q t+q t^{2}$ & $q t+q^{2} t+q^{3} t^{2}$ & $q^{2} t+q^{3} t+q^{3} t^{2}$ & $1+q^{2} t^{2}$ \\
\hline$(0 ; 2,2)$ & $t^{4}$ & $t^{2}$ & $t^{3}+q t^{3}+q t^{4}$ & $t+q t^{2}+q t^{3}$ & $t+q t+q t^{2}$ & $t^{2}+q^{2} t^{4}$ \\
\hline$(2 ; 1,1)$ & $t^{3}$ & $q^{2} t^{3}$ & $t+t^{2}+q t^{3}$ & $q t+q^{2} t^{2}+q^{2} t^{3}$ & $q^{2} t+q^{2} t^{2}+q^{3} t^{3}$ & $t+q t^{2}$ \\
\hline$(1 ; 2,1)$ & $t^{4}$ & $q t^{3}$ & $t^{2}+t^{3}+q t^{4}$ & $t+q t^{2}+q^{2} t^{4}$ & $q t+q t^{2}+q^{2} t^{3}$ & $t^{2}+q t^{3}$ \\
\hline$(0 ; 2,1,1)$ & $t^{6}$ & $t^{3}$ & $t^{4}+t^{5}+q t^{6}$ & $t^{2}+t^{3}+q t^{4}$ & $t+t^{2}+q t^{3}$ & $t^{4}+q t^{5}$ \\
\hline$\left(1 ; 1^{3}\right)$ & $t^{6}$ & $q t^{6}$ & $t^{3}+t^{4}+t^{5}$ & $t^{3}+q t^{4}+q t^{5}$ & $q t^{3}+q t^{4}+q t^{5}$ & $t^{2}+t^{4}$ \\
\hline$\left(0 ; 1^{4}\right)$ & $t^{10}$ & $t^{6}$ & $t^{7}+t^{8}+t^{9}$ & $t^{4}+t^{5}+t^{7}$ & $t^{3}+t^{4}+t^{5}$ & $t^{6}+t^{8}$ \\
\hline
\end{tabular}

\begin{tabular}{l|c|c|c|c|c|c|} 
& $(0 ; 2,2)$ & $(2 ; 1,1)$ & $(1 ; 2,1)$ & $(0 ; 2,1,1)$ & $\left(1 ; 1^{3}\right)$ & $\left(0 ; 1^{4}\right)$ \\
\hline$(4 ;)$ & $q^{6}+q^{8}$ & $q^{3}+q^{4}+q^{5}$ & $q^{4}+q^{5}+q^{7}$ & $q^{7}+q^{8}+q^{9}$ & $q^{6}$ & $q^{10}$ \\
\hline$(0 ; 4)$ & $q^{2}+q^{4}$ & $q^{3} t+q^{4} t+q^{5} t$ & $q^{3}+q^{4} t+q^{5} t$ & $q^{3}+q^{4}+q^{5}$ & $q^{6} t$ & $q^{6}$ \\
\hline$(3 ; 1)$ & $q^{4}+q^{5} t$ & $q+q^{2}+q^{3} t$ & $q^{2}+q^{3}+q^{4} t$ & $q^{4}+q^{5}+q^{6} t$ & $q^{3}$ & $q^{6}$ \\
\hline$(1 ; 3)$ & $q^{2}+q^{3} t$ & $q t+q^{2} t+q^{3} t^{2}$ & $q+q^{2} t+q^{4} t^{2}$ & $q^{2}+q^{3}+q^{4} t$ & $q^{3} t$ & $q^{4}$ \\
\hline$(0 ; 3,1)$ & $q+q^{2} t$ & $q t^{2}+q^{2} t^{2}+q^{3} t^{3}$ & $q t+q^{2} t^{2}+q^{3} t^{2}$ & $q+q^{2}+q^{3} t$ & $q^{3} t^{2}$ & $q^{3}$ \\
\hline$(2 ; 2)$ & $q^{2}+q^{4} t^{2}$ & $q+q t+q^{2} t$ & $q+q^{2} t+q^{3} t$ & $q^{3}+q^{3} t+q^{4} t$ & $q^{2}$ & $q^{4}$ \\
\hline$(0 ; 2,2)$ & $1+q^{2} t^{2}$ & $q t^{2}+q t^{3}+q^{2} t^{3}$ & $q t+q t^{2}+q^{2} t^{3}$ & $q+q t+q^{2} t$ & $q^{2} t^{2}$ & $q^{2}$ \\
\hline$(2 ; 1,1)$ & $q^{2} t+q^{3} t^{2}$ & $1+q t+q t^{2}$ & $q+q t+q^{2} t^{2}$ & $q^{2}+q^{3} t+q^{3} t^{2}$ & $q$ & $q^{3}$ \\
\hline$(1 ; 2,1)$ & $q t+q^{2} t^{2}$ & $t+q t^{2}+q t^{3}$ & $1+q t^{2}+q^{2} t^{3}$ & $q+q^{2} t+q^{2} t^{2}$ & $q t$ & $q^{2}$ \\
\hline$(0 ; 2,1,1)$ & $t+q t^{2}$ & $t^{3}+q t^{4}+q t^{5}$ & $t^{2}+q t^{3}+q t^{4}$ & $1+q t+q t^{2}$ & $q t^{3}$ & $q$ \\
\hline$\left(1 ; 1^{3}\right)$ & $q t^{2}+q t^{4}$ & $t+t^{2}+t^{3}$ & $t+t^{2}+q t^{3}$ & $q t+q t^{2}+q t^{3}$ & 1 & $q$ \\
\hline$\left(0 ; 1^{4}\right)$ & $t^{2}+t^{4}$ & $t^{5}+t^{6}+t^{7}$ & $t^{3}+t^{5}+t^{6}$ & $t+t^{2}+t^{3}$ & $t^{4}$ & 1 \\
\hline
\end{tabular}

presented in [7], the transpose symbol (prime) is missing; these correspond to Tables 1-5.

An example illustrating the first symmetry property (227) is (cf. Table 7)

$$
K_{(2,0 ; 1,1)(2,0 ; 2)}(q, t)=q+q t+q^{2} t=K_{(3,0 ; 1)(2,0 ; 2)}(t, q),
$$


Table $7: K(q, t)^{\prime}$ for degree $(4 \mid 2)$

\begin{tabular}{l|c|c|c|c|c|c|c|c|c|} 
& $(4,0 ;)$ & $(3,1 ;)$ & $(3,0 ; 1)$ & $(1,0 ; 3)$ & $(2,0 ; 2)$ & $(2,1 ; 1)$ & $(2,0 ; 1,1)$ & $(1,0 ; 2,1)$ & $\left(1,0 ; 1^{3}\right)$ \\
\hline$(4,0 ;)$ & 1 & $q+q^{2}$ & $q+q^{2}+q^{3}$ & $q^{3}$ & $q^{2}+q^{4}$ & $q^{3}$ & $q^{3}+q^{4}+q^{5}$ & $q^{4}+q^{5}$ & $q^{6}$ \\
\hline$(3,1 ;)$ & $q t$ & $1+q^{2} t$ & $q+q^{2} t+q^{3} t$ & $q^{4} t$ & $q^{2}+q^{3} t$ & $q$ & $q^{2}+q^{3}+q^{4} t$ & $q^{3}+q^{5} t$ & $q^{4}$ \\
\hline$(3,0 ; 1)$ & $t$ & $t+q t$ & $1+q t+q^{2} t$ & $q^{2}$ & $q+q^{2} t$ & $q$ & $q+q^{2}+q^{3} t$ & $q^{2}+q^{3}$ & $q^{3}$ \\
\hline$(1,0 ; 3)$ & $t^{2}$ & $q t^{2}+q^{2} t^{3}$ & $t+q t^{2}+q^{2} t^{2}$ & 1 & $q t+q^{2} t^{2}$ & $q^{3} t^{3}$ & $q t+q^{2} t+q^{3} t^{2}$ & $q+q^{2} t$ & $q^{3} t$ \\
\hline$(2,0 ; 2)$ & $t^{2}$ & $t+q t^{2}$ & $t+q t+q t^{2}$ & $q t$ & $1+q^{2} t^{2}$ & $q t$ & $q+q t+q^{2} t$ & $q+q^{2} t$ & $q^{2}$ \\
\hline$(2,1 ; 1)$ & $q t^{3}$ & $t+q t^{2}$ & $q t+q t^{2}+q^{2} t^{3}$ & $q^{3} t^{3}$ & $q t+q^{2} t^{2}$ & 1 & $q+q^{2} t+q^{2} t^{2}$ & $q^{2} t+q^{3} t^{2}$ & $q^{2}$ \\
\hline$(2,0 ; 1,1)$ & $t^{3}$ & $t^{2}+t^{3}$ & $t+t^{2}+q t^{3}$ & $q t$ & $t+q t^{2}$ & $t^{2}$ & $1+q t+q t^{2}$ & $q+q t$ & $q$ \\
\hline$(1,0 ; 2,1)$ & $t^{4}$ & $t^{3}+q t^{5}$ & $t^{2}+t^{3}+q t^{4}$ & $t$ & $t^{2}+q t^{3}$ & $q t^{4}$ & $t+q t^{2}+q t^{3}$ & $1+q t^{2}$ & $q t$ \\
\hline$\left(1,0 ; 1^{3}\right)$ & $t^{6}$ & $t^{4}+t^{5}$ & $t^{3}+t^{4}+t^{5}$ & $t^{3}$ & $t^{2}+t^{4}$ & $t^{3}$ & $t+t^{2}+t^{3}$ & $t+t^{2}$ & 1 \\
\hline
\end{tabular}


since $(2,0 ; 1,1)^{\prime}=(3,0 ; 1)$ and $(2,0 ; 2)$ is self-conjugate. An example of the relation $(228)$ is

$$
K_{(2,0 ; 1,1)(1,0 ; 2,1)}(q, t)=t+q t^{2}+q t^{3}=q t^{5} K_{(3,0 ; 1)(1,0 ; 2,1)}\left(q^{-1}, t^{-1}\right) .
$$

The factor $t^{5}$ can be checked as follows (the diagram of $\Lambda$ is filled with numbers that add up to $d(\Lambda)$ while the skew diagram $\mathcal{S} \Lambda$, obtained from $\Lambda^{\circledast}$ by dropping the squares marked by a $\times$, is filled by numbers that add up to $n(\mathcal{S} \Lambda))$ :

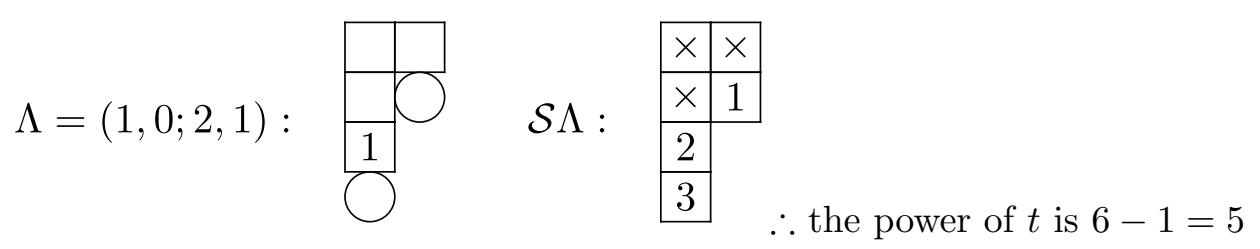

Similarly, for $q$ factor, we have

$$
\begin{aligned}
& \Lambda^{\prime}=(3,1 ; 0): \\
& \begin{array}{|l|l|l|}
\hline 0 & & 0 \\
\hline 0 & & \\
\hline
\end{array} \\
& \mathcal{S} \Lambda^{\prime}: \begin{array}{|l|l|l|l|}
\hline \times & \times & 0 & 0 \\
\hline \times & 1 & \multicolumn{3}{|c}{} \\
\cline { 1 - 1 } &
\end{array} \\
& \therefore \text { the power of } q \text { is } 1-0=1
\end{aligned}
$$

\subsection{Two new conjectures for the Kostka coefficients}

We conclude this section with the formulation of two remarkable conjectures that relate the generalized coefficients $K_{\Omega \Lambda}(q, t)$ of fermionic degree $m=1$ to the usual $(q, t)$-Kostka coefficients.

Conjecture 25. Let $\Lambda$ be a superpartition of fermionic degree $m=1$, and let $J_{\Lambda}$ be the integral form of the Macdonald superpolynomial. Let also $\psi$ be the linear application that maps $S_{\Omega}$ to $S_{\Omega \circledast}$. Then

$$
\psi\left(J_{\Lambda}\right)=J_{\Lambda \circledast .}
$$

This conjecture implies that the usual $(q, t)$-Kostka coefficient $K_{\mu \lambda}(q, t)$ of two partitions $\mu, \lambda$ can be calculated from their lower-degree super-relatives as

$$
K_{\mu \lambda}(q, t)=\sum_{\Omega \mid \Omega^{\circledast}=\mu} K_{\Omega \Lambda}(q, t),
$$


where $\Lambda$ is any superpartition that can be obtained from $\lambda$ by replacing a square by a circle and the sum is over all $\Omega$ 's that can be obtained from $\mu$ by replacing a square by a circle. Moreover, the expression for the sum on the right-hand side is independent of the choice of $\Lambda$. We thus relate a Kostka coefficient of degree $(n \mid 0)$ to a sum of degree $(n-1 \mid 1)$ Kostka coefficients. In other words, the $(n-1 \mid 1)$ Kostka coefficients provide a refinement of the usual $(q, t)$-Kostka coefficients.

For instance, consider $\lambda=\mu=(3,1)$. There are two ways of replacing a square by a circle:

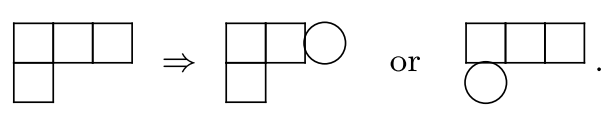

Choosing $\Lambda=(2 ; 1)$, we have [7]

$$
\begin{aligned}
J_{(2 ; 1)}= & t S_{(3 ;)}+q^{2} t S_{(0 ; 3)}+(1+q t) S_{(2 ; 1)}+q(1+q t) S_{(1 ; 2)} \\
& +q^{2}(1+q t) S_{(0 ; 2,1)}+q S_{(1 ; 1,1)}+q^{3} S_{(0 ; 1,1,1)},
\end{aligned}
$$

so that, using (233),

$$
K_{(2 ; 1)(2 ; 1)}+K_{(0 ; 3)(2 ; 1)}=(1+q t)+q^{2} t=K_{(3,1)(3,1)} .
$$

The same result follows by taking $\Lambda=(0 ; 3)$ :

$$
\begin{aligned}
J_{(0 ; 3)}= & t S_{(3 ;)}+S_{(0 ; 3)}+q t(1+q) S_{(2 ; 1)}+q(1+q t) S_{(1 ; 2)} \\
& +q(1+q) S_{(0 ; 2,1)}+q^{3} t S_{(1 ; 1,1)}+q^{3} S_{(0 ; 1,1,1)},
\end{aligned}
$$

which implies that

$$
K_{(2 ; 1)(0 ; 3)}+K_{(0 ; 3)(0 ; 3)}=\left(q t+q^{2} t\right)+1=K_{(3,1)(3,1)} .
$$

To formulate the second conjecture, we need to introduce the notion of a concatenable superpartition, defined as one for which $\Lambda_{m}^{a} \geq \Lambda_{1}^{s}$. Such a superpartition can be transformed into a partition $\lambda$ by removing the semi-coma: $\lambda=\left(\Lambda^{a}, \Lambda^{s}\right)$. For instance, $(5,3 ; 2,1,1)$ is concatenable and the corresponding partition is $\lambda=(5,3,2,1,1)$.

Conjecture 26. Let $\Lambda$ be a concatenable superpartition of fermionic degree $m=1$, and let $\lambda$ be its corresponding partition. Let also $\phi$ be the linear application that maps $S_{\Omega}$ to $S_{\mu}$ if $\Omega$ is concatenable (and $\mu$ its corresponding partition) and to zero otherwise. Then

$$
\phi\left(J_{\Lambda}\right)=J_{\lambda}
$$


If $\Lambda$ and $\Omega$ are two $m=1$ concatenable superpartitions whose corresponding partitions are $\lambda$ and $\mu$, this implies that:

$$
K_{\Lambda \Omega}=K_{\lambda \mu}
$$

For instance, considering $\phi\left(J_{(2 ; 1)}\right)$ given in $(235)$, one recovers the expression of $J_{(2,1)}$ :

$$
J_{(2,1)}=q S_{(1,1,1)}+(q t+1) S_{(2,1)}+t S_{(3)} .
$$

\section{Appendix A. Schur polynomials in superspace}

In this section, we prove that the limits of the Macdonald polynomials $P_{\Lambda}(x ; q, t)$, as $q=t \rightarrow 0$ and $q=t \rightarrow \infty$, are well defined; they provide two new families of Schur polynomials in superspace, namely $s_{\Lambda}(x)$ and $\bar{s}_{\Lambda}(x)$, whose existence was only conjectured in [7]. The proof almost immediately follows from (33) (see lemma 27 below), which expresses a Macdonald superpolynomial in terms of non-symmetric Macdonald polynomials $E_{\eta}(x ; q, t)$, and from Ion's work $[16,17]$, which shows that a non-symmetric Macdonald polynomial (for an affine root system) can be interpreted, when $t \rightarrow 0, \infty$, as the character of a certain Demazure module, and more particularly, proves the regularity of $E_{\eta}(x ; q, t)$ as $q=t \rightarrow 0, \infty$.

It is worth noting that the characters of the Demazure modules were also studied from an algebro-combinatorial point of view by $\mathrm{Fu}$ and Lascoux [15]. They showed that the character formulas can be written in terms of key polynomials $\mathrm{K}_{\eta}(x)$ and $\hat{\mathrm{K}}_{\eta}(x)$ (see [15, Section 4] for a precise definition). Combining the results of [17] and [15] on the irreducible root system of type $A$, one readily obtains the explicit relation between the limiting non-symmetric Macdonald polynomials (of type $A$ ) and the key polynomials:

$$
E_{\eta}(x ; 0,0)=\widehat{\mathrm{K}}_{\eta}(x), \quad E_{\eta}(x ; \infty, \infty)=\mathrm{K}_{\omega_{\mathbf{n}} \eta}\left(\omega_{\mathbf{n}} x\right)
$$

where $\eta=\left(\eta_{1}, \eta_{2}, \ldots, \eta_{n}\right)$ is a composition, $\omega_{\mathbf{n}} \eta=\left(\eta_{n}, \eta_{n-1}, \ldots, \eta_{1}\right)$ and $\left(\omega_{\mathbf{n}} x\right)=\left(x_{n}, \ldots, x_{1}\right)$.

Lemma 27. The Macdonald superpolynomials $P_{\Lambda}(x, \theta ; q, t)$ can also be written in terms of the non-symmetric Macdonald polynomials as

$$
P_{\Lambda}=c_{\Lambda}^{\prime}(t) \sum_{\sigma \in S_{N} /\left(S_{m} \times S_{m^{c}}\right)} \mathcal{K}_{\sigma}\left(\theta_{1} \cdots \theta_{m} A_{m} U_{m^{c}}^{+} E_{\left(\left(\Lambda^{a}\right)^{R}, \Lambda^{s}\right)}\right)
$$


where

$$
c_{\Lambda}^{\prime}(t)=\frac{(-1)^{\left(\begin{array}{c}
m \\
2
\end{array}\right)}}{f_{\Lambda^{s}}(t)} .
$$

Proof. This expression differs from (33) in that we consider here the composition obtained from the concatenation of $\left(\left(\Lambda^{a}\right)^{R}, \Lambda^{s}\right)$ instead of its (fully) reversed version. To fix the normalization coefficient, we follow exactly the proof of Proposition 7 except that equation (90) is now replaced by

$$
\left[x^{\Lambda}\right] \quad \sum_{w \in S_{m^{c}}} T_{w} x^{\Lambda}=\sum_{w \in S_{m^{c}} \mid w(\Lambda)=\Lambda} t^{\ell(w)} .
$$

We need to consider all those permutations that leaves $\Lambda^{s}$ fixed, which gives

$$
\left[x^{\Lambda}\right] \quad \sum_{w \in S_{m^{c}}} T_{w} x^{\Lambda}=\prod_{i=0}^{\Lambda_{1}^{s}}\left(\sum_{w^{(i)} \in S_{n_{\Lambda^{s}}(i)}} t^{\ell\left(w^{(i)}\right)}\right)=\prod_{i=0}^{\Lambda_{1}^{s}}\left[n_{\Lambda^{s}}(i)\right]_{t} !=f_{\Lambda^{s}}(t) .
$$

We thus have, as expected,

$$
\left[m_{\Lambda}\right] P_{\Lambda}=c_{\Lambda}^{\prime}(t)(-1)^{\left(\begin{array}{c}
m \\
2
\end{array}\right)} f_{\Lambda^{s}}(t)=1 .
$$

Proposition 28. The Schur superpolynomials,

$$
s_{\Lambda}(x, \theta):=P_{\Lambda}(x, \theta ; 0,0), \quad \text { and } \quad \bar{s}_{\Lambda}(x, \theta)=P_{\Lambda}(x, \theta ; \infty, \infty),
$$

are well-defined symmetric polynomials in superspace. Furthermore, the Schur superpolynomials can be expressed in terms of the key polynomials (see (250) and (252) below).

Proof. We first set $q=t$ in (243) and take the limit $t \rightarrow 0$. Given that $E_{\eta}(x ; 0,0)$ is well-defined, we only need to evaluate $c_{\Lambda}^{\prime}(t)$ and $U_{m^{c}}^{+}$as $t \rightarrow 0$. Giving that $[0]_{t} !=1$ and $\lim _{t \rightarrow 0}[n]_{t}=\lim _{t \rightarrow 0} \frac{1-t^{n}}{1-t}=1$, we have $f_{\Lambda^{s}}(0)=1$. Moreover,

$$
\lim _{t \rightarrow 0} T_{i}=\frac{-x_{i+1}}{x_{i}-x_{i+1}}\left(K_{i, i+1}-1\right)=\widehat{\pi}_{i} .
$$


Consequently,

$$
\begin{aligned}
& \lim _{t \rightarrow 0} P_{\Lambda}(x, \theta ; t, t) \\
& =\lim _{t \rightarrow 0} c_{\Lambda}^{\prime}(t) \sum_{\sigma \in S_{N} /\left(S_{m} \times S_{m^{c}}\right)} \mathcal{K}_{\sigma} \theta_{1} \cdots \theta_{m} A_{m} \sum_{w \in S_{m^{c}}} T_{w}(t) E_{\left(\left(\Lambda^{a}\right)^{R}, \Lambda^{s}\right)}(x ; t, t) \\
& =(-1)^{\left(\begin{array}{c}
m \\
2
\end{array}\right)} \sum_{\sigma \in S_{N} /\left(S_{m} \times S_{m^{c}}\right)} \mathcal{K}_{\sigma} \theta_{1} \cdots \theta_{m} A_{m} \widehat{\Pi}_{m^{c}} \widehat{E}_{\left(\left(\Lambda^{a}\right)^{R}, \Lambda^{s}\right)}(x ; 0,0)
\end{aligned}
$$

where $\widehat{\Pi}_{m^{c}}=\sum_{w \in S_{m^{c}}} \widehat{\pi}_{w}$. Thus, according to $(242)$,

$$
P_{\Lambda}(x, \theta ; 0,0)=(-1)^{\left(\begin{array}{c}
m \\
2
\end{array}\right)} \sum_{\sigma \in S_{N} /\left(S_{m} \times S_{m^{c}}\right)} \mathcal{K}_{\sigma} \theta_{1} \cdots \theta_{m} A_{m} \widehat{\Pi}_{m^{c}} \widehat{\mathbf{K}}_{\left(\left(\Lambda^{a}\right)^{R}, \Lambda^{s}\right)}(x) .
$$

This shows that the Schur superpolynomial $s_{\Lambda}(x, \theta)$ is well-defined.

For the second family of Schur superpolynomials $\bar{s}_{\Lambda}(x, \theta)$, let us first write the $t$-symmetrizer $U^{+}$as follows (see [27, eq. (2.25)]):

$$
\begin{aligned}
U_{m^{c}}^{+} f(x) & =\frac{1}{\Delta_{m^{c}}} A_{m^{c}}\left(\prod_{m<i<j}\left(x_{i}-t x_{j}\right) f(x)\right) \\
& =\frac{t^{n(n-1) / 2}}{\Delta_{m^{c}}} A_{m^{c}}\left(\prod_{m<i<j}\left(\frac{1}{t} x_{i}-x_{j}\right) f(x)\right),
\end{aligned}
$$

where $n=N-m$. Then, we rewrite $f_{\Lambda^{s}}(t)$ as

$$
\begin{aligned}
f_{\Lambda^{s}}(t)=\prod_{i=1}^{\Lambda_{1}^{s}}\left[n_{\Lambda^{s}}(i)\right]_{t} ! & =\prod_{i=1}^{\Lambda_{1}^{s}} t^{n_{\Lambda^{s}}(i)\left(n_{\Lambda^{s}}(i)-1\right) / 2}\left[n_{\Lambda^{s}}(i)\right]_{1 / t^{\prime}} ! \\
& =t^{\sum_{i} n_{\Lambda^{s}}(i)\left(n_{\Lambda^{s}}(i)-1\right) / 2} f_{\Lambda^{s}}(1 / t) .
\end{aligned}
$$

For $\mu=\left(\mu_{1}, \mu_{2}, \ldots, \mu_{N}\right)$, with $\mu_{N} \geq 0$, we define

$$
\operatorname{inv}(\mu)=N(N-1) / 2-\sum_{i} n_{\mu}(i)\left(n_{\mu}(i)-1\right) / 2
$$

In words, given all possible pairs of parts of $\mu$, if we subtract all the pairs formed between repeated parts, we get the inversion number. By combining 
these formulas with (33) and (40), we get

$$
\begin{aligned}
\lim _{t \rightarrow \infty} & P_{\Lambda}(x ; t, t) \\
= & \lim _{t \rightarrow \infty} c_{\Lambda}(t) \sum_{\sigma \in S_{N} /\left(S_{m} \times S_{m^{c}}\right)} \mathcal{K}_{\sigma} \theta_{1} \cdots \theta_{m} A_{m} U_{m^{c}}^{+} E_{\Lambda^{R}}(x ; t, t) \\
= & \lim _{t \rightarrow \infty} \frac{(-1)^{\left(\begin{array}{c}
m \\
2
\end{array}\right) t^{n(n-1) / 2}}}{t^{n(n-1) / 2-\operatorname{inv}\left(\Lambda^{s}\right)} f_{\Lambda^{s}}(1 / t) t^{\operatorname{inv}\left(\Lambda^{s}\right)}} \\
& \times \lim _{t \rightarrow \infty} \sum_{\sigma \in S_{N} /\left(S_{m} \times S_{m}\right)} \mathcal{K}_{\sigma} \theta_{1} \cdots \theta_{m} A_{m} \frac{1}{\Delta_{m^{c}}} A_{m^{c}} \prod_{m<i<j}\left(\frac{1}{t} x_{i}-x_{j}\right) E_{\Lambda^{R}}(x ; t, t)
\end{aligned}
$$

which leads to

$$
\begin{array}{r}
\lim _{t \rightarrow \infty} P_{\Lambda}(x ; t, t)=(-1)^{\left(\begin{array}{c}
m \\
2
\end{array}\right)} \sum_{\sigma \in S_{N} /\left(S_{m} \times S_{m^{c}}\right)} \mathcal{K}_{\sigma} \theta_{1} \cdots \theta_{m} A_{m} \frac{1}{\Delta_{m^{c}}} A_{m^{c}}(-1)^{\left(\begin{array}{c}
n \\
2
\end{array}\right)} \\
x_{m+1}^{0} x_{m+2}^{1} \cdots x_{N}^{n-1} E_{\Lambda^{R}}(x ; \infty, \infty) .
\end{array}
$$

Finally, using $(242)$ and $\delta^{(n)}=(n-1, \ldots, 1,0, \ldots)$, we obtain

(252) $\quad P_{\Lambda}(x ; \infty, \infty)=$

$$
(-1)^{\left(\begin{array}{c}
m \\
2
\end{array}\right)+\left(\begin{array}{c}
n \\
2
\end{array}\right)} \sum_{\sigma \in S_{N} /\left(S_{m} \times S_{m^{c}}\right)} \mathcal{K}_{\sigma} \theta_{1} \cdots \theta_{m} A_{m} \frac{1}{\Delta_{m^{c}}} A_{m^{c}}(\omega x)^{\delta^{(n)}} \mathrm{K}_{\left(\Lambda^{s}, \Lambda^{a}\right)}(\omega x),
$$

which shows that $\bar{s}_{\Lambda}(x, \theta)$ is well-defined.

We recall that the Macdonald superpolynomials are stable with respect to the number $N$ of indeterminates. The same stability holds for $s_{\Lambda}=s_{\Lambda}(x, \theta)$ and $\bar{s}_{\Lambda}=\bar{s}_{\Lambda}(x, \theta)$, since they are limits of the Macdonald superpolynomials. This allows us to consider $s_{\Lambda}$ and $\bar{s}_{\Lambda}$ as elements of the algebra $\mathscr{R}$ of symmetric functions in superspace (see Section 5 ). The next corollary shows that within this context, there is a natural duality between $\bar{s}_{\Lambda}$ and $s_{\Lambda}$.

Corollary 29. Let $\langle\langle\mid\rangle\rangle$ and $\omega$ respectively denote the scalar product $\langle\langle\mid\rangle\rangle_{q, t}$ with $q=t=1$, and the homomorphism defined in (170). Moreover, let

$$
s_{\Lambda}^{*}=\omega \bar{s}_{\Lambda^{\prime}} .
$$

Then,

$$
\left\langle\left\langle s_{\Lambda}^{*} \mid s_{\Omega}\right\rangle\right\rangle=\delta_{\Lambda, \Omega}
$$


Equivalently,

$$
\prod_{i, j}\left(1-x_{i} y_{j}-\theta_{i} \phi_{j}\right)^{-1}=\sum_{\Lambda}(-1)^{\left(\begin{array}{c}
m \\
2
\end{array}\right)} s_{\Lambda}^{*}(x, \theta) s_{\Lambda}(y, \phi) .
$$

Proof. The first result is obtained by taking the limit $q, t \rightarrow 0$ in (181). The second result follows from (254) and [11, Lemma 37].

\section{Appendix B. Proof of a combinatorial identity}

The equivalence, as $N$ goes to $\infty$, between the two norms in (221) relies on the equality

$$
\lim _{N \rightarrow \infty} \frac{d_{\left(\Lambda^{a R}, \Lambda^{s}\right)}^{\prime} d_{\Lambda^{s} R}}{\left.d_{\left(\Lambda^{a}, \Lambda^{s}\right.}\right)}=\frac{h_{\Lambda^{s}} h_{\Lambda}^{\uparrow}}{h_{\Lambda}^{\downarrow}} .
$$

The limit needs to appear on the right-hand side since there is a residual dependence upon $N$ in the ratio $d_{\Lambda^{s R}} / d_{\left(\Lambda^{a}, \Lambda^{s R}\right)}$ (the reversed partition $\Lambda^{s R}$ contains $N-m-\ell\left(\Lambda^{s}\right)$ zeros).

We first introduce, as in [19], a convenient decomposition of the leglengths of a composition $\gamma$ :

$$
l(s)=l^{\uparrow}(s)+l^{\downarrow}(s)
$$

with

$$
\begin{aligned}
& l^{\uparrow}(s)=\#\left\{k=1, \ldots, i-1 \mid j \leq \gamma_{k}+1 \leq \gamma_{i}\right\}, \\
& l^{\downarrow}(s)=\#\left\{k=i+1, \ldots, N \mid j \leq \gamma_{k} \leq \gamma_{i}\right\} .
\end{aligned}
$$

In order to better visualize expressions $l^{\uparrow}(s)$ and $l^{\downarrow}(s)$, we put (as in [20]) a symbol at the end of each row of $\gamma$, here trading the French hexagon for a triangle, e.g.,

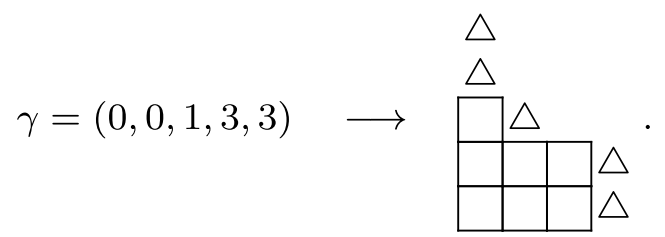

For $s=(i, j), l^{\uparrow}(s)$ is given by the number of triangles above row $i$ in columns $j^{\prime}$ for $j \leq j^{\prime} \leq \gamma_{i}$ (e.g., $l^{\uparrow}((4,1))=3$ in the above example), while $l^{\downarrow}(s)$ is given by the number of triangles below row $i$ in columns $j^{\prime}$ for $j+1 \leq j^{\prime} \leq \gamma_{i}+1$ (e.g., $l^{\downarrow}((4,1))=1$ in the above example). 
Lemma 30. Identity (256) is equivalent to

$$
\frac{d_{\left(\Lambda^{a R}, \Lambda^{s}\right)}^{\prime}}{h_{\Lambda^{s}}}\left[\frac{d_{\Lambda^{s} R}}{d_{\left(\Lambda^{a}, \Lambda^{s R}\right)}}\right]_{0}=\frac{h_{\Lambda}^{\uparrow}}{h_{\Lambda}^{\downarrow}}
$$

where

$$
\left[\frac{d_{\Lambda^{s} R}}{d_{\left(\Lambda^{a}, \Lambda^{s R}\right)}}\right]_{0}=\frac{d_{\Lambda^{s} R}}{d_{\left(\Lambda^{a}, \Lambda^{s} R\right.}}\left(\prod_{(i, 1) \in \Lambda^{s}} \frac{d_{\Lambda^{s} R}(s)}{d_{\left(\Lambda^{a}, \Lambda^{s} R\right)}(s)}\right)^{-1}
$$

that is, the products corresponding the the cells in the first columns of $\Lambda^{s}$ were removed from $d_{\Lambda^{s R}} / d_{\left(\Lambda^{a}, \Lambda^{s R}\right)}$.

Proof. We first isolate the part of $d_{\Lambda^{s R}} / d_{\left(\Lambda^{a}, \Lambda^{s R}\right)}$ that depends upon $N$ :

$$
\lim _{N \rightarrow \infty} \frac{d_{\Lambda^{s R}}}{\left.d_{\left(\Lambda^{a}, \Lambda^{s} R\right.}\right)}=\left[\frac{d_{\Lambda^{s} R}}{d_{\left(\Lambda^{a}, \Lambda^{s} R\right.}}\right]_{0} \lim _{N \rightarrow \infty} \prod_{(i, 1) \in \Lambda^{s}} \frac{d_{\Lambda^{s} R}(s)}{d_{\left(\Lambda^{a}, \Lambda^{s} R\right.}(s)} .
$$

It thus suffices to prove that

$$
\lim _{N \rightarrow \infty} \prod_{(i, 1) \in \Lambda^{s}} \frac{d_{\Lambda^{s} R}(s)}{d_{\left(\Lambda^{a}, \Lambda^{s} R\right.}(s)}=1 .
$$

The last equality is rather clear: it is a ratio of terms of the form $1-$ $q^{a(s)+1} t^{l(s)+1}$ for which the leg-length $l(s)$ tends to infinity as $N$ goes to infinity (the number of triangles above $(i, 1) \in \Lambda^{s}$ goes to infinity). Take for instance the case $\Lambda=(2,1 ; 2,1)$ and introduce $M$ zeros represented by $\triangle^{M}$ :

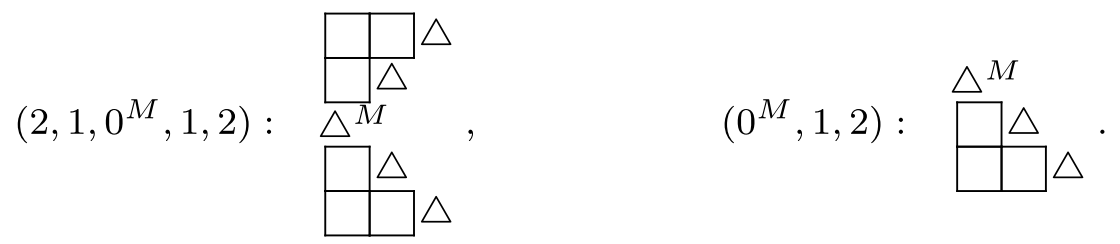

The ratio on the left-hand side becomes

(265) $\lim _{M \rightarrow \infty} \prod_{(i, 1) \in \Lambda^{s}} \frac{d_{\Lambda^{s} R}(s)}{d_{\left(\Lambda^{a}, \Lambda^{s R}\right.}(s)}=\lim _{M \rightarrow \infty} \frac{\left(1-q t^{M+1}\right)\left(1-q^{2} t^{M+2}\right)}{\left(1-q t^{M+1}\right)\left(1-q^{2} t^{M+3}\right)}=1$. 
Proposition 31. Identity (260) holds.

Proof. First, observe that the identity (256) is satisfied identically (for all $N$ actually) when $\Lambda^{a}=\emptyset$ and $\Lambda^{s}=\mu$, since (266)

l.h.s. $(256)=\lim _{N \rightarrow \infty} \frac{d_{\mu}^{\prime} d_{\mu^{R}}}{d_{\mu^{R}}}=d_{\mu}^{\prime} \quad$ and $\quad$ r.h.s. $(256)=\frac{h_{\mu} h_{\mu}^{\uparrow}}{h_{\mu}^{\downarrow}}=\frac{h_{\mu} d_{\mu}^{\prime}}{h_{\mu}}=d_{\mu}^{\prime}$.

The result is thus true in the case $\Lambda^{a}=\emptyset$. We thus suppose by induction that (260) holds for some $\Lambda=\left(\Lambda^{a} ; \Lambda^{s}\right)$ and the aim is to prove that $(260)$ is still valid if we add a fermionic row $b>\Lambda_{1}^{a}$ to obtain $\tilde{\Lambda}=\left(\tilde{\Lambda}^{a} ; \Lambda^{s}\right)$, where $\tilde{\Lambda}^{a}=\left(b, \Lambda_{1}^{a}, \Lambda_{2}^{a}, \ldots\right)$. Defining

$$
\Delta F(\Lambda)=\frac{F(\tilde{\Lambda})}{F(\Lambda)}
$$

it thus suffices to demonstrate that

$$
\Delta \text { l.h.s. }(260)=\Delta \text { r.h.s. }(260) \text {. }
$$

Given that $\tilde{\Lambda}^{s}=\Lambda^{s}$, the factors $d_{\Lambda^{s} R}$ and $h_{\Lambda^{s}}$ are not affected by the transformation $\Lambda \rightarrow \tilde{\Lambda}$. Hence

$$
\boldsymbol{\Delta} \text { l.h.s. }(260)=\boldsymbol{\Delta}\left(\frac{d_{\left(\Lambda^{a R}, \Lambda^{s}\right)}^{\prime}}{\left[d_{\left(\Lambda^{a}, \Lambda^{s R}\right)}\right]_{0}}\right)
$$

Finally, (268) will follow from the two relations

$$
(1): \Delta d_{\left(\Lambda^{a}, \Lambda^{s}\right)}^{\prime}=\boldsymbol{\Delta} \bar{h}_{\Lambda}^{\uparrow} \quad \text { and } \quad(2): \boldsymbol{\Delta}\left[d_{\left(\Lambda^{a}, \Lambda^{s R}\right)}\right]_{0}=\boldsymbol{\Delta} \bar{h}_{\Lambda}^{\downarrow}
$$

where $\bar{h}^{\uparrow \downarrow}$ is defined as $h^{\uparrow \downarrow}$ except that the product runs over all squares of $\Lambda$ :

$$
\bar{h}_{\Lambda}^{\uparrow}=\prod_{s \in \Lambda}\left(1-q^{a_{\Lambda^{*}}(s)+1} t^{l_{\Lambda} \circledast(s)}\right) \quad \text { and } \quad \bar{h}_{\Lambda}^{\downarrow}=\prod_{s \in \Lambda}\left(1-q^{a_{\Lambda} \circledast(s)} t^{l_{\Lambda^{*}}(s)+1}\right) .
$$

Observe that,

$$
\frac{\bar{h}_{\Lambda}^{\uparrow}}{\bar{h}_{\Lambda}^{\downarrow}}=\frac{h_{\Lambda}^{\uparrow}}{h_{\Lambda}^{\downarrow}}
$$

and thus, as claimed, we only have to prove the two relations (270). 
There are two types of contributions to $\Delta$ : those corresponding to the modifications to the leg-lengths of the squares of $\Lambda^{s}$ in rows of length larger than $b$, denoted by $\boldsymbol{\Delta}_{1}$, and those corresponding to the squares of the added fermionic row, denoted by $\boldsymbol{\Delta}_{2}$. They will be treated separately.

Consider first the variation $\boldsymbol{\Delta}_{1}$ and the relation (1). In this case, $d_{\gamma}^{\prime} \rightarrow d_{\tilde{\gamma}}^{\prime}$ where $\gamma=\left(\Lambda^{a R}, \Lambda^{s}\right)=$ and $\tilde{\gamma}=\left(\Lambda^{a R}, b, \Lambda^{s}\right)=\left(\left(\tilde{\Lambda}^{a}\right)^{R}, \Lambda^{s}\right)$. The leg-lengths of the squares in columns $1 \leq j \leq b+1$ and rows $\gamma_{i}>b$ is increased by 1 by adding the new fermionic row. We have thus:

$$
\boldsymbol{\Delta}_{1} d_{\gamma}^{\prime}=\prod_{\substack{s=(i, j) \in \gamma \\ \gamma_{i}>b \\ 1 \leq j \leq b+1}} \frac{1-q^{a_{\gamma}(s)+1} t^{l_{\gamma}(s)+1}}{1-q^{a_{\gamma}(s)+1} t^{l_{\gamma}(s)}}
$$

where we indicated explicitly with respect to which diagram the arm- and leg-lengths are calculated. Consider now the corresponding variation of $h^{\uparrow}$. Since the expression of $\bar{h}^{\uparrow}$ involves $l_{\Lambda^{\circledast}}$, the addition of the fermionic row of size $b$ increases by 1 the leg-length of the squares in column $1 \leq j \leq b+1$ of rows $\Lambda_{i}^{s}>b$ :

$$
\Delta_{1} \bar{h}_{\Lambda}^{\uparrow}=\prod_{\substack{s=(i, j) \in \Lambda^{s} \\ \Lambda_{i} \\ 1 \leq b b \\ 1 \leq b+1}} \frac{1-q^{a_{\Lambda^{*}}(s)+1} t^{l_{\Lambda \circledast}(s)+1}}{1-q^{a_{\Lambda^{*}}(s)+1} t^{l} \Lambda(s)} .
$$

In order to compare expressions (273) and (274), we need to clarify the meaning of the entries in each product. Note that in the first product, the rows $\gamma_{i}>b$ are such that $\gamma_{i}=\Lambda_{i^{\prime}}^{s}$ for some $i^{\prime}$. The product is thus over the same number of squares in the two cases. Now, in reordering the rows of $\gamma$ to get $\Lambda^{*}$ (an operation that does not affect the arm-lengths), we readily see that $a_{\gamma}=a_{\Lambda^{*}}$ in (273). Next, to compare the leg-lengths, we note that for $s=(i, j)$, the leg-length $l_{\gamma}(s)=l_{\gamma}^{\downarrow}(s)+l_{\gamma}^{\uparrow}(s)$ in $(273)$ is such that

$$
l_{\gamma}^{\downarrow}=\#\left\{k>i^{\prime} \mid j \leq \Lambda_{k}^{s}\right\}
$$

and

$$
l_{\gamma}^{\uparrow}=\#\left\{k<i \mid j \leq \gamma_{k}+1 \leq \gamma_{i}\right\}=\#\left\{k \mid j \leq \tilde{\Lambda}_{k}^{a}+1\right\} .
$$

The sum $l_{\gamma}^{\uparrow}+l_{\gamma}^{\downarrow}$ thus corrresponds exactly to the definition of $l_{\Lambda \circledast}$ in (274). This demonstrates the equivalence of relations (273) and (274). 
Consider next relation (2), focusing again on the contribution $\boldsymbol{\Delta}_{1}$. Let us first obtain the variation resulting from $d_{\eta} \rightarrow d_{\tilde{\eta}}$, where $\eta=\left(\Lambda^{a}, \Lambda^{s R}\right)$ and $\tilde{\eta}=\left(b, \Lambda^{a}, \Lambda^{s R}\right)$. The analysis of $\boldsymbol{\Delta}_{1} d_{\eta}$ is similar to the one above for $\boldsymbol{\Delta}_{1} d_{\gamma}^{\prime}$, except that we need to keep in mind that the contribution of the first column is not considered anymore. We find

$$
\boldsymbol{\Delta}_{1}\left[d_{\eta}\right]_{0}=\prod_{\substack{s=(i, j) \in \eta \\ \eta_{i}>b \\ 2 \leq j \leq b+1}} \frac{1-q^{a_{\eta}(s)+1} t^{l_{\eta}(s)+2}}{1-q^{a_{\eta}(s)+1} t^{l_{\eta}(s)+1}} .
$$

Let us now turn to the corrresponding variation in $\bar{h}^{\downarrow}$. The expression for $\bar{h}^{\downarrow}$ involves $a_{\Lambda \circledast}$ which is the same as $a_{\Lambda^{*}}$ for a bosonic row. Also, since the leg-length entering in $\bar{h}^{\uparrow}$ is $l_{\Lambda^{*}}$, which does not count the possible circle at the end of the column, the addition of the fermionic row of length $b$ increases by 1 the leg-lengths of the squares in columns $1 \leq j \leq b$ (and not $b+1$ ) of the rows $\Lambda_{i}^{s}>b$ :

$$
\boldsymbol{\Delta}_{1} \bar{h}_{\Lambda}^{\downarrow}=\prod_{\substack{s=(i, j) \in \Lambda^{s} \\ \Lambda_{i}^{s}>b \\ 1 \leq j \leq b}} \frac{1-q^{a_{\Lambda^{*}}(s)} t^{l_{\Lambda^{*}}(s)+2}}{1-q^{a_{\Lambda^{*}}(s)} t^{l_{\Lambda^{*}}(s)+1}} .
$$

Before we can do a direct comparison between (277) and (278), we have to perform the substitution $j \rightarrow j+1$ in (277). Since $a_{\eta}(i, j+1)+1=a_{\eta}(i, j)$, this gives

$$
\boldsymbol{\Delta}_{1}\left[d_{\eta}\right]_{0}=\prod_{\substack{s=(i, j+1) \in \eta \\ \eta_{i}>b \\ 1 \leq j \leq b}} \frac{1-q^{a_{\eta}(i, j)} t^{l_{\eta}(s)+2}}{1-q^{a_{\eta}(i, j)} t^{l_{\eta}(s)+1}}
$$

Letting $\eta_{i}=\Lambda_{i^{\prime}}^{s}$, we see that the powers of $q$ are identical in both expressions. Moreover, since the block $\Lambda^{a}$ still lies in the upper position of the composition, we can write the leg-length $l_{\eta}(i, j+1)$, using eq. (258), as (280)

$l_{\eta}(i, j+1)=\#\left\{k \mid j \leq \Lambda_{k}^{a}\right\}+\#\left\{k>i^{\prime} \mid j \leq \Lambda_{k}^{s}<\Lambda_{i^{\prime}}^{s}\right\}+\#\left\{k<i^{\prime} \mid \Lambda_{k}^{s}=\Lambda_{i^{\prime}}^{s}\right\}$,

which corresponds, up to a reordering of the parts of $\Lambda^{s}$ of the same size as $\Lambda_{i^{\prime}}^{s}$, precisely to the expression of $l_{\Lambda^{*}}$ in $(278)$. We have thus verified the equivalence of (277) and (278). 
Consider now the variation $\boldsymbol{\Delta}_{2}$, namely, the contribution of the squares of the added fermionic row of size $b$. As before, we let $\gamma=\left(\Lambda^{a R}, \Lambda^{s}\right)$, $\tilde{\gamma}=\left(\Lambda^{a R}, b, \Lambda^{s}\right)$ and $\tilde{\Lambda}=\left(\tilde{\Lambda}^{a} ; \Lambda^{s}\right)$ with $\tilde{\Lambda}^{a}=\left(b, \Lambda_{1}^{a}, \Lambda_{2}^{a}, \ldots\right)$. If the added fermionic row of size $b$ corresponds to $\tilde{\gamma}_{i}$ (resp. $\left.\tilde{\Lambda}_{i^{\prime}}\right)$ in $\tilde{\gamma}($ resp. $\tilde{\Lambda})$, the verification of relation (1) amounts to compare (281)

$$
\boldsymbol{\Delta}_{2} d_{\gamma}^{\prime}=\prod_{1 \leq j \leq b}\left[1-q^{b-j+1} t^{l_{\tilde{\gamma}}(i, j)}\right] \quad \text { and } \quad \boldsymbol{\Delta}_{2} \bar{h}_{\Lambda}^{\uparrow}=\prod_{1 \leq j \leq b}\left[1-q^{b-j+1} t^{l_{\tilde{\Lambda} \circledast}^{\left(i^{\prime}, j\right)}}\right] .
$$

The powers of $q$ are manifestly the same in the two contributions. The expression for the leg-length on the l.h.s. is

$$
l_{\tilde{\gamma}}(i, j)=l^{\uparrow}(i, j)+l^{\downarrow}(i, j),
$$

where

$$
l^{\uparrow}(i, j)=\#\left\{k \mid j \leq \Lambda_{k}^{a}+1\right\} \quad \text { and } \quad l^{\downarrow}(i, j)=\#\left\{k \mid j \leq \Lambda_{k}^{s} \leq b\right\} .
$$

This form of $l_{\tilde{\gamma}}(i, j)$ is clearly the same as the expression of $l_{\tilde{\Lambda} \circledast}\left(i^{\prime}, j\right)$ on the r.h.s, which demonstrates the equivalence of the two variations in (281).

It only remains to establish the correctness of relation (2) under $\boldsymbol{\Delta}_{2}$. Recall that $\eta=\left(\Lambda^{a}, \Lambda^{s R}\right)$ and $\tilde{\eta}=\left(b, \Lambda^{a}, \Lambda^{s R}\right)$. If the added fermionic row of size $b$ corresponds to $\tilde{\Lambda}_{i}$ in $\tilde{\Lambda}$, the verification of relation (2) amounts to compare

$$
\boldsymbol{\Delta}_{2} d_{\eta}=\prod_{1 \leq j \leq b}\left[1-q^{b-j+1} t^{l_{\tilde{\eta}}(1, j)+1}\right]
$$

and

$$
\boldsymbol{\Delta}_{2} h_{\Lambda}^{\downarrow}=\prod_{1 \leq j \leq b}\left[1-q^{b-j+1} t^{l_{\Lambda^{*}}(i, j)+1}\right]
$$

where we used the fact that $a_{\tilde{\Lambda}_{\circledast}}(i, j)=a_{\tilde{\Lambda}^{*}}(i, j)+1$ given that row $i$ of $\tilde{\Lambda}$ is fermionic. Again the powers of $q$ match. We have

$$
l_{\tilde{\eta}}(1, j)=l^{\downarrow}(1, j)=\#\left\{k \mid j \leq \Lambda_{k}^{a} \leq b \text { or } j \leq \Lambda_{k}^{s} \leq b\right\},
$$

which corresponds to $l_{\tilde{\Lambda}^{*}}(i, j)$ on the r.h.s. This completes the demonstration of the two relations (270) and thus of Proposition 31. 
Let us consider an example: $\Lambda=(0 ; 4,1)$ and $\tilde{\Lambda}=(2,0 ; 4,1)$. We have thus added a fermionic row of length 2 . We ignore the zeros of $\Lambda^{s}$ given that they contribute only to the first column which is removed. The triangle at the end of the added row is written in parenthesis and the circle of the added row is marked by a $\times$ : (287)

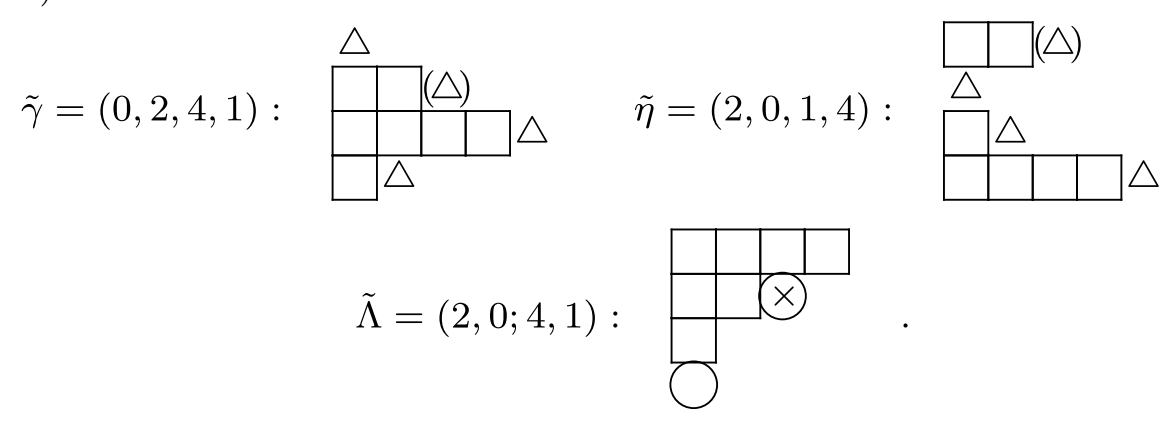

We have thus, for the product of the two variations $\boldsymbol{\Delta}_{1}$ and $\boldsymbol{\Delta}_{2}$, (288)

$$
\begin{aligned}
& \frac{d_{(0,2,4,1)}^{\prime}}{d_{(0,4,1)}^{\prime}}=\frac{\left(1-q^{4} t^{3}\right)\left(1-q^{3} t\right)\left(1-q^{2} t\right)}{\left(1-q^{4} t^{2}\right)\left(1-q^{3}\right)\left(1-q^{2}\right)} \times\left(1-q^{2} t^{2}\right)(1-q)=\frac{h_{(2,0 ; 4,1)}^{\uparrow}}{h_{(0 ; 4,1)}^{\uparrow}} \\
& \frac{d_{(2,0,1,4)}}{d_{(0,1,4)}}=\frac{\left(1-q^{3} t^{3}\right)\left(1-q^{2} t^{2}\right)}{\left(1-q^{3} t^{2}\right)\left(1-q^{2} t\right)} \times\left(1-q^{2} t\right)(1-q)=\frac{h_{(2,0 ; 4,1)}^{\downarrow}}{h_{(0 ; 4,1)}^{\downarrow}} .
\end{aligned}
$$

\section{References}

[1] T. H. Baker, C. F. Dunkl, and P. J. Forrester, Polynomial eigenfunctions of the Calogero-Sutherland-Moser models with exchange terms, in J. F. van Diejen and L. Vinet, Calogero-Sutherland-Moser Models, CRM Series in Mathematical Physics, Springer, 2000, pp. 37-42. MR1843561

[2] T. H. Baker and P. J. Forrester, A $q$-analogue of the type $A$ Dunkl operator and integral kernel, Int. Math. Res. Not. 148 (1997), 667-686. MR1460388

[3] W. Baratta, Some properties of Macdonald polynomials with prescribed symmetry, Kyushu J. Math. 64 (2010), 323-343. MR2744627

[4] F. Bergeron, Algebraic Combinatorics and Coinvariant Spaces, CMS Treatise in Mathematics, A.K. Peters Publishers, 2009. MR2538310 
[5] F. Bergeron and A. M. Garsia, Science fiction and Macdonald's polynomials, in Algebraic Methods and q-Special Functions (Montreal, QC, 1996), CRM Proc. Lecture Notes, Vol. 22, Amer. Math. Soc., Providence, RI, 1999, pp. 1-52. MR1726826

[6] D. Bernard, M. Gaudin, F. D. Haldane, and V. Pasquier, Yang-Baxter equation in long range interacting system, J. Phys. A A26, (1993) 5219-5236. MR1248713

[7] O. Blondeau-Fournier, P. Desrosiers, L. Lapointe, and P. Mathieu, Macdonald polynomials in superspace: conjectural definition and positivity conjectures, Lett. Math. Phys. 101 (2012), 27-47. MR2935476

[8] I. Cherednik, Non-symmetric Macdonald polynomials, Int. Math. Res. Notices 10 (1995), 483-515. MR1358032

[9] P. Desrosiers, L. Lapointe, and P. Mathieu, Supersymmetric CalogeroMoser-Sutherland models and Jack superpolynomials, Nucl. Phys. B606 (2001), 547-582. MR1847975

[10] P. Desrosiers, L. Lapointe, and P. Mathieu, Jack polynomials in superspace, Commun. Math. Phys. 242 (2003), 331-360. MR2018276

[11] P. Desrosiers, L. Lapointe, and P. Mathieu, Classical symmetric functions in superspace, J. Alg. Comb. 24 (2006), 209-238. MR2259017

[12] P. Desrosiers, L. Lapointe, and P. Mathieu, Orthogonality of Jack polynomials in superspace, Adv. Math. 212 (2007), 361-388. MR2319773

[13] P. Desrosiers, L. Lapointe, and P. Mathieu, Evaluation and normalization of Jack polynomials in superspace, Int. Math. Res. Notices $\mathbf{2 3}$ (2012), 5267-5327.

[14] P. Desrosiers, L. Lapointe, and P. Mathieu, Jack superpolynomials with negative fractional parameter: clustering properties and super-Virasoro ideals, Commun. Math. Phys. 316 (2012), 395-440. MR2993921

[15] A. M. Fu and A. Lascoux, Non-symmetric Cauchy kernels for the classical groups, J. of Comb. Th. A 116 (2009), 903-917. MR2513641

[16] B. Ion, Demazure characters and non-symmetric Macdonald polynomials, Duke Math. J. 116 (2003), 299-318. MR1953294

[17] B. Ion, Standard bases for affine parabolic modules and nonsymmetric Macdonald polynomials, J. Alg. 319 (2008), 3480-3517. MR2408328 
[18] A. N. Kirillov and M. Noumi, Affine Hecke algebras and raising operators for Macdonald polynomials, Duke Math. J. 93 (1998), 1-39. MR1620075

[19] F. Knop and S. Sahi, A recursion and a combinatorial formula for Jack polynomials, Invent. Math. 128 (1997), 9-22. MR1437493

[20] L. Lapointe, Y. Le Borgne, and P. Nadeau, A normalization formula for the Jack polynomials in superspace and an identity on partitions, Electronic J. Comb. 16 (2009) Article \#R70. MR2515747

[21] A. Lascoux, Symmetric Functions and Combinatorial Operators on Polynomials, CBMS, Vol. 99, AMS, 2003. MR2017492

[22] A. Lascoux, Private communication (January 2012).

[23] A. Lascoux and M.P. Schützenberger, Keys and standard bases, in Invariant Theory and Tableaux, IMA Volumes in Math and its Applications, Vol. 19, Springer, 1988, pp. 125-144. MR1035493

[24] I. G. Macdonald, Symmetric Functions and Hall Polynomials, 2nd ed. Clarendon Press, 1995. MR1354144

[25] I. G. Macdonald, Affine Hecke Algebras and Orthogonal Polynomials, Séminaire Bourbaki 1994-1995, exposé 797, pp. 189-207. MR1423624

[26] I. G. Macdonald, Affine Hecke Algebras and Orthogonal Polynomials, Cambridge Univ. Press (2003). MR1976581

[27] D. Marshall, Symmetric and non-symmetric Macdonald polynomials, Ann. Comb. 3 (1999), 385-415. MR1772356

[28] K. Mimachi and M. Noumi, A reproducing kernel for nonsymmetric Macdonald polynomials, Duke Math. J. 91 (1998), 621-634. MR1604183

[29] E. M. Opdam, Harmonic analysis for certain representations of graded Hecke algebras, Acta Math. 175 (1995), 75-121. MR1353018

[30] S. Sahi, A new scalar product for nonsymmetric Jack polynomials, Internat. Math. Res. Notices 20 (1996), 997-1004. MR1422372

[31] R. P. Stanley, Some combinatorial properties of Jack symmetric functions, Adv. Math. 77 (1988), 76-115. MR1014073 
Olivier Blondeau-Fournier

DÉPARTEMENT DE Physique, DE GÉNIE PHySique ET D'optique UNIVERSITÉ LAVAL

QUÉBEC, G1V 0A6

CANADA

E-mail address: olivier.b-fournier.1@ulaval.ca

PATRICK DesRosiers

Instituto de Matemática y Física

UNIVERSIDAD DE TALCA

2 NORTE 685 , TALCA

CHILE

E-mail address: patrick.desrosiers@inst-mat.utalca.cl

LuC LAPOINTE

Instituto de Matemática y Física

UNIVERSIDAD DE TALCA

2 NORTE 685 , TALCA

CHILE

E-mail address: lapointe@inst-mat.utalca.cl

Pierre Mathieu

DÉPARTEMENT DE PHYSIQUe, DE GÉNIE PHYSIQUe ET D'OPTIQUe UNIVERSITÉ LAVAL

QuÉBEC, G1V 0A6

CANADA

E-mail address: pmathieu@phy.ulaval.ca

Received January 31, 2012 